\title{
Pore-spanning lipid membranes as a tool to study membrane permeabilization
}

\section{by antimicrobial peptides}

\author{
Dissertation \\ for the award of the degree \\ “Doctor rerum naturalium" (Dr. rer. nat.) \\ of the Georg-August-University Göttingen \\ within the doctoral program \\ Physics of Biological and Complex Systems \\ of the Georg-August-University School of Science (GAUSS)
}

submitted by

Henrik Neubacher

from Minden

Göttingen, 2017 


\section{Thesis COMMITTEe}

Prof. Dr. Claudia Steinem (Reviewer)

Institute for Organic and Biomolecular Chemistry, Georg-August-Universität Göttingen

Prof. Dr. Reinhard Jahn (Reviewer)

Department of Neurobiology, Max Planck Institute for Biophysical Chemistry, Göttingen

Prof. Dr. Jörg Enderlein

Third Institute of Physics, Georg-August Universität Göttingen

\section{AdDitional Members OF THE EXAMination BoARD}

Dr. Jochen Hub

Computational Molecular Biophysics Group, Department of Molecular Structural Biology, Georg-August-Universität Göttingen

Prof. Dr. Michael Meinecke

European Neuroscience Institute, Georg-August-Universität Göttingen

Prof. Dr. Tim Salditt

Institute for X-Ray Physics, Georg-August-Universität Göttingen

DATE OF ORAL EXAMINATION: March $9^{\text {th }} 2017$ 
I, Henrik Neubacher, hereby certify that my doctoral thesis entitled "Pore-spanning lipid membranes as a tool to study membrane permeabilization by antimicrobial peptides" has been written independently and with no other sources and aids than quoted.

Göttingen, January $15^{\text {th }} 2017$

Henrik Neubacher 
Parts of this work have been published:

Neubacher H, Mey I, Carnarius C, Lazzara TD, Steinem C.: Permeabilization assay for antimicrobial peptides based on pore-spanning lipid membranes on nanoporous alumina, Langmuir. 2014 Apr 29;30(16):4767-74 [2] 


\section{Abstract}

Antimicrobial peptides (AMPs) have a high therapeutic potential in combating microbial diseases. Their modes of action are still not well understood, and screening methods to identify and improve AMPs are needed. This work explores the use of pore-spanning lipid membranes as a model system to assay AMP activity, and to investigate their modes of action. Different porous substrates are tested and evaluated for their suitability for chipbased dye translocation assays that monitor the ability of an aqueous dye to move across a lipid bilayer.

Three porous substrates with different porosity, optical and surface characteristics were tested: open pore silicon nitride, closed pore silica, and closed pore anodic aluminium oxide (AAO) substrates. Lipid bilayer patches were created by spreading dye-doped giant unilamellar vesicles on the porous surface. The spreading process and membrane characteristics were characterized using confocal laser scanning microscopy in a timeresolved manner. Using the AAO support, a dye inflow assay was established. The AMPs melittin and magainin-2 were tested for their effects on lipid bilayers by monitoring dye translocation and changes in membrane fluorescence in response to administration of the respective AMP. Qualitative and quantitative measurements of the changes in membrane permeability gave insights into possible modes of action. Results suggest that melittin, but not magainin-2, acts in part by removing material from the membrane. Meanwhile, magainin-2 but not melittin is able to form pores whose size correlates with the concentration of the peptide. Finally, the assay indicated a preference of magainin- 2 and $\delta$ lysin for the liquid disordered phase of phase-separated membranes.

With this, the ground work was laid for the development of an assay suitable to screen compounds for AMP activity, efficiency and efficacy, specificity and toxicity. The setup can also be used to investigate details of the modes of action of different AMPs. It has the potential for an on-chip screening platform for high throughput purposes, and applications beneficial for the in vitro research on new antimicrobial agents. 



\section{Zusammenfassung}

Antimikrobielle Peptide (AMPs) haben ein hohes therapeutisches Potential bei der Bekämpfung mikrobieller Krankheiten. Ihre Wirkungsweisen sind immer noch nicht vollständig aufgeklärt, und Screening-Methoden zur Identifizierung und Verbesserung von AMPs werden benötigt. Diese Arbeit untersucht porenüberspannende Membranen als potenzielles Testsystem für die Prüfung von Substanzen auf AMP-Aktivität sowie die Erforschung der Wirkungsweise von AMPs. Verschiedene feste Trägerstoffe wurden auf ihre Eignung für Chip-basierte Farbstoff-Translokationstests geprüft. Hierbei wurde die Fähigkeit eines AMPs, die Lipidmembran zu perforieren, mit Hilfe eines wasserlöslichen Farbstoffes gezeigt.

Drei Trägerstoffe wurden getestet: offenporige Siliziumdioxid-, geschlossenporige Siliziumnitrid- sowie geschlossenporige Substrate aus anodischem Aluminiumoxid (AAO). Porenüberspannende Membranen wurden durch Spreiten von farbstoffdotierten giant unilammellar vesicles erzeugt. Der Spreitprozess sowie die Eigenschaften der gebildeten planaren porenüberspannenden Membranen wurden mit Hilfe von konfokaler LaserScanning-Fluoreszenzmikroskopie untersucht. Die Parameter für eine robuste Überwachung in räumlicher als auch Zeitdimension wurden erarbeitet. Mit AAO als Trägersubstanz wurde ein Farbstoff-Einströmungstest etabliert. Mit diesem Test wurde untersucht, auf welche Weise die AMPs Melittin und Magainin-2 die Membran verändern. Erkenntnisse darüber wurden anhand der Beobachtung von Membranfluoreszenz und Durchlässigkeit für verschieden große wässrige Farbstoffe gewonnen. Qualitative und quantitative Untersuchungen der Veränderung der Membrandurchlässigkeit gaben Einblicke in mögliche Wirkungsweisen der untersuchten AMPs. Die Ergebnisse legen nahe, dass Melittin, nicht aber Magainin-2,seine Wirkung zum Teil durch Entfernen von Membranmaterial entfaltet. Im Gegensatz dazu zeigte Magainin-2, nicht aber Melittin, eine konzentrationsabhängige Variabilität in der Größe geformter Poren. Schließlich konnte gezeigt werden dass Magainin-2 und $\delta$-Lysin in phasenseparierten Membranen die liquid disordered Phase bevorzugen.

In dieser Arbeit wurden die Forschungsgrundlagen für die Entwicklung eines Tests zur systematischen Suche nach AMPs bezüglich Effizienz und Wirksamkeit, Spezifität und Toxizität gelegt. Die Untersuchungsmethode erlaubt die Erforschung der Wirkungsweisen verschiedener AMPs. Damit hat die hier etablierte Methode das Potenzial für einen Chipbasierten Schnelltest auf die antimikrobiellen Eigenschaften von Kandidatensubstanzen. 



\section{Contents}

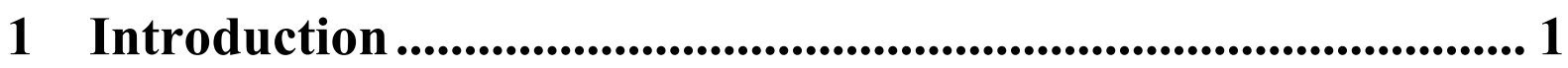

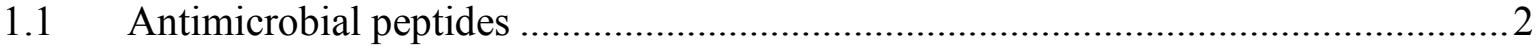

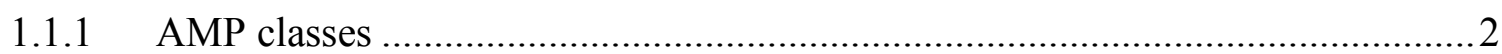

1.1.2 AMP modes of action on the membrane ..................................................... 3

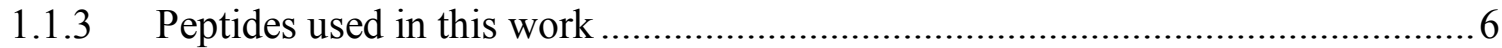

1.2 Model membrane systems for the characterization of AMPs................................. 7

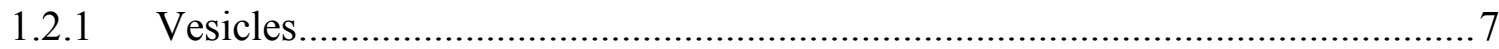

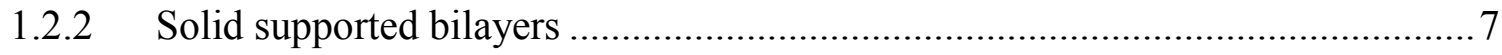

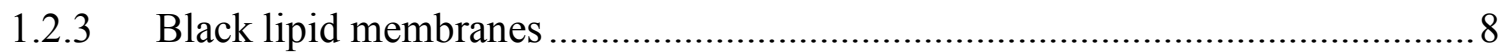

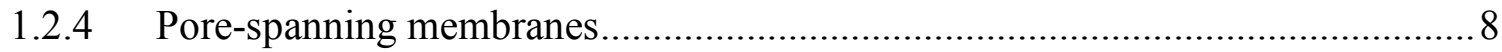

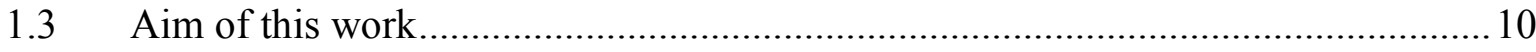

2 Materials and Methods ........................................................... 11

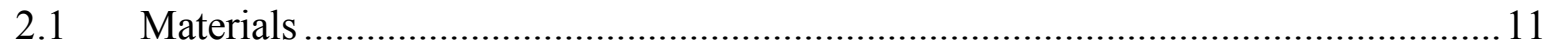

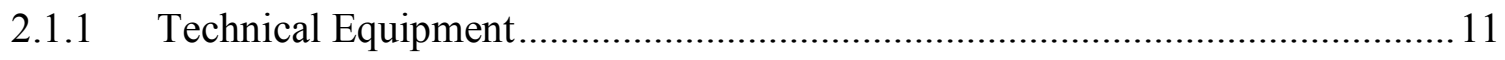

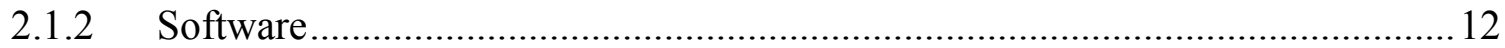

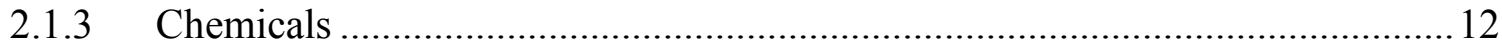

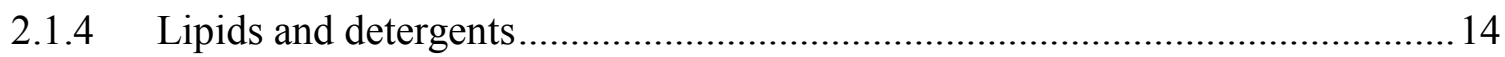

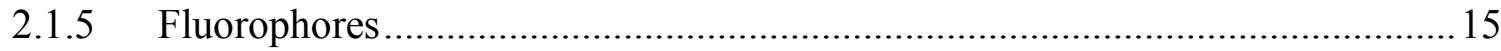

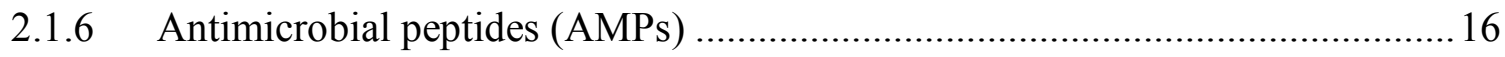

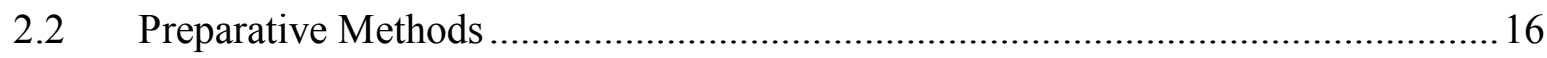

2.2.1 Giant unilamellar vesicle (GUV) preparation ............................................ 17

2.2.2 Preparation of nanoporous anodic aluminium oxide (AAO) ........................... 18

2.2.3 Preparation of silicon-based porous substrates............................................29

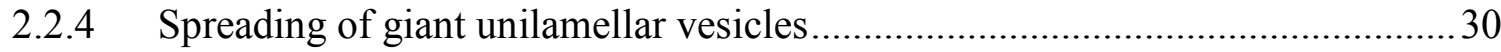

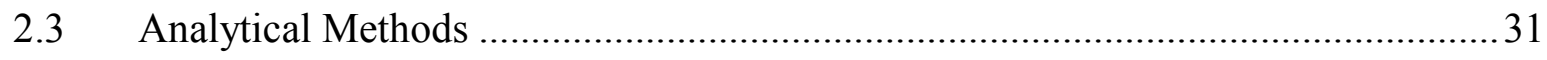

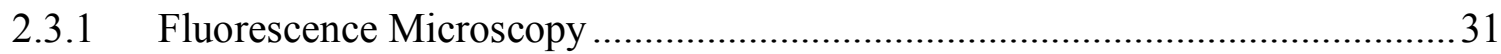




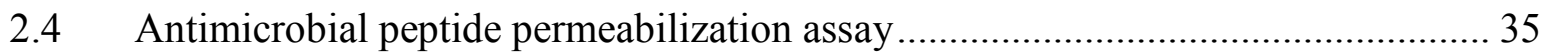

2.4.1 Membrane preparation and localization for fluorescence microscopy …........... 36

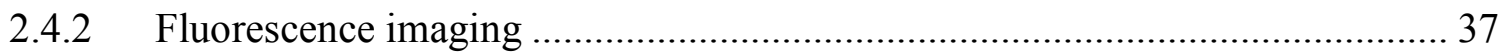

2.4.3 Microscope setup and detection parameters …........................................... 37

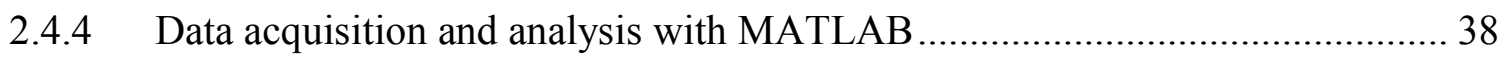

\section{Results............................................................................... 41}

3.1 Pore-spanning membrane systems on open silicon nitride pores .......................... 41

3.2 Pore-spanning membrane systems on closed silica pores.................................... 43

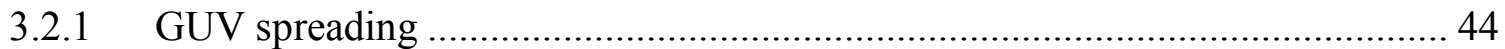

3.2.2 Membrane behavior and stability after GUV spreading ................................. 45

3.2.3 Detailed assessment of dye release on closed silica pores .............................. 47

3.3 Pore-spanning membranes on AAO support: Investigation of AMPs .................... 49

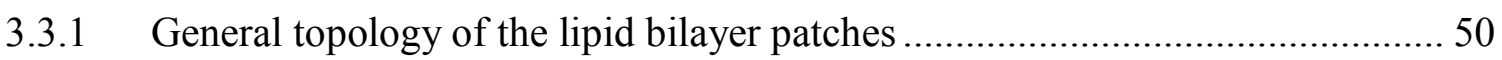

3.3.2 Determination of a suitable data acquisition mode ....................................... 52

3.3.3 Changes in membrane and cavity fluorescence after permeabilization:

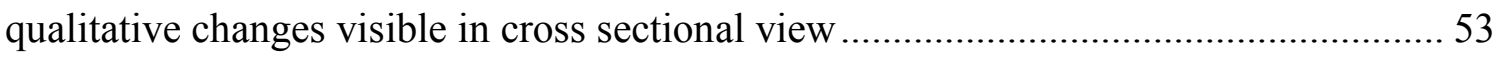

3.3.4 Changes in membrane and cavity fluorescence after permeabilization: qualitative changes visible in $2 \mathrm{D}$ imaging of the $\mathrm{xy}$ plane

3.3.5 Quantitative analysis of fluorescence changes upon membrane permeabilization 58

3.4 Investigation of AMP permeabilization modes using kinetic fluorescence analyses ..

3.4.1 Kinetics analysis of lipid bilayer permeabilization through magainin-2 .......... 62

3.4.2 Kinetics analysis of lipid bilayer permeabilization through melittin................ 68

3.4.3 Comparison of melittin and magainin-2 results ........................................... 72

3.5 Phase-separated membranes on AAO support...................................................... 73

3.5.1 Preparation of phase-separated membranes on AAO support …..................... 73

3.5.2 Spreading of phase separated vesicles in dependency on temperature and lipid amount

3.5.3 AMP interaction with specific lipid membrane phases 78 


\section{Discussion}

4.1 Characteristics of lipid bilayer formation on porous substrates …........................ 85

4.1.1 GUV spreading and planar membrane formation: A detailed view ...................85

4.1.2 Closed pores may lead to spherical membrane perturbations 86

4.2 Monitoring material exchange across the membrane: dye release and dye inflow assays

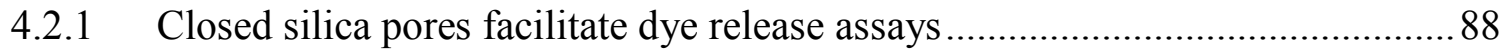

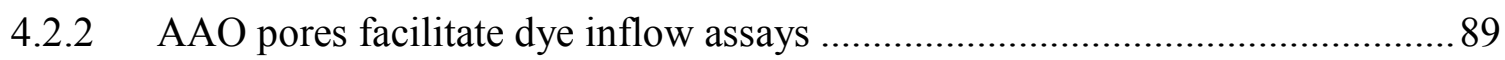

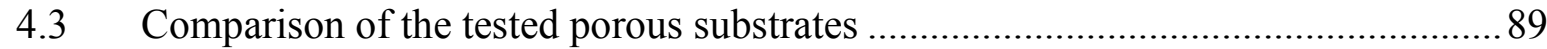

4.4 Investigation of AMP modes of action on pore-spanning membranes ................... 90

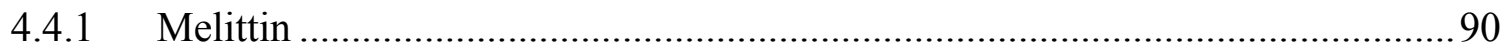

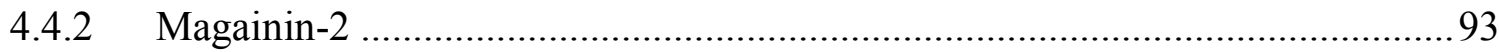

4.4.3 Comparison of melittin and magainin-2 actions on PSM ............................... 95

4.4.4 Considerations on the overall versatility of PSM assays to investigate AMP action

4.5 AMPs preferably act on the $1_{d}$ phase of pore-spanning phase-separated membranes ..

4.6 Comparison of pore-spanning membranes with other artificial membrane systems 97

4.6.1 Advantages and challenges of pore-spanning membranes ............................... 97

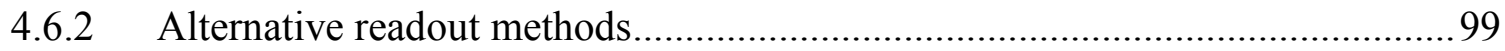

4.7 Potential applications of pore-spanning membrane assays ................................. 100

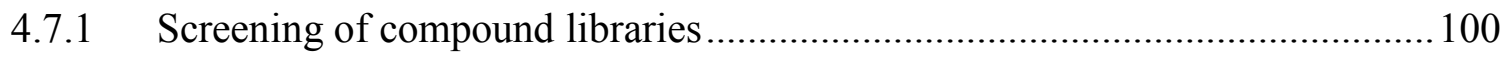

4.7.2 Assessing AMP toxicity towards pathogens versus host cells ........................ 100

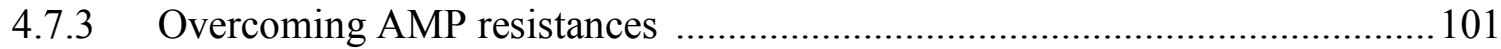

\section{Appendix .............................................................................................. 103}

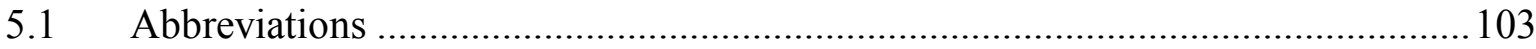

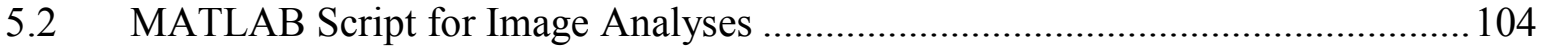

6 Acknowledgements............................................................................ 113

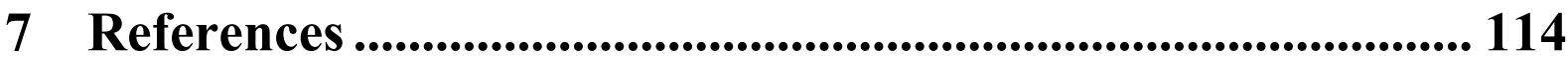


Contents

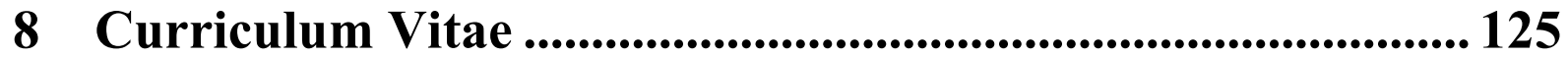




\section{Introduction}

Multiresistent bacteria have increased manifold over the last decade [3]. This has been attributed to the increased use of antibiotics in animal farming and in human healthcare either to treat infections or due to the increased use of immunosuppressive anti-cancer therapies. Fewer and fewer new antibiotic substances are found, and resistances are on the rise [4]. This is why it is imperative to develop new substances with antimicrobial activity and tolerable effects against the cells of the patient.

The discovery of antibiotics was a milestone in the history of medicine. Antibiotics are biomolecules that inhibit the growth of microorganisms or kill them. Through the use of antibiotics as drugs it was now possible to treat many bacterial infectious diseases, which could previously only be treated symptomatically. Many antibiotics are metabolic products of fungi or bacteria and serve as a defense mechanism against other microorganisms.

Antimicrobially active substances can also be found on the skin of higher organisms like mammals, reptiles or amphibians. These substances are part of the innate immune system and act as a first line defense against microorganisms found virtually in every organism [5].

Antibiotics can be classified by the target of antimicrobial activity. They can either inhibit metabolic processes or protein biosynthesis by targeting involved enzymes, or directly target the bacterial cell walls and cell membranes [6][7].

A very prominent class of antibiotics are $\beta$-lactam antibiotics that also include penicillins. These antibiotics are very efficient and target the bacterial cell wall. However, bacteria may develop a resistance to these antibiotics by synthesizing a $\beta$-lactamase that can hydrolyze the $\beta$-lactam ring and therefore inactivate the antibiotic [8].

Antibiotic resistance is an ever current issue as a lot of bacteria have gradually developed resistances against various currently available antibiotics. The development of novel antibiotics is a big challenge in the near future. For this reason, research in the field of antimicrobial peptides (AMPs) is of increasing interest. Various classes of antimicrobial peptides are known to directly target bacterial cell membranes, and some are already commercially available as antibiotics. One prominent example is tyrothricin, a mixture of gramicidines and tyrocidines from Bacillus brevis [9]. Amongst its uses is the local application as a gel under the trade name Tyrosur [10], or with additional compounds in lozenges as Dorithricin [11]. A long-term goal of the research on potential AMPs is the design of novel therapeutic agents based on antimicrobial peptides as alternatives to currently available antibiotics. 
For the design of AMPs as novel therapeutic agents it is essential to understand their modes of interaction on cellular lipid bilayers. More detailed knowledge may lead to the ability to design and select novel agents that could become useful for the antimicrobial treatment in patients, both human and livestock.

\subsection{Antimicrobial peptides}

Antimicrobial peptides are a class of naturally occurring antibiotics that possess activity against a variety of pathogens [12][5]. While these peptides are elements of the innate immune system of many different organisms, only few resistances are known [7]. They primarily act on the plasma membrane of pathogens, although a variety of further targets has been reported (see 1.1.2).

\subsubsection{AMP classes}

Antimicrobial peptides are classically short-chain cationic peptides of up to 40 amino acids length and a molecular mass of less than $10 \mathrm{kDa}$. While certain structure homologies are apparent, sequence homology is limited. AMPs can be largely placed in four structural groups (Fig. 1.1, [13][5],[13][7]). Even though longer polypeptides and proteins with antimicrobial properties have also been described [14][15], this work focuses on a few classical short-chain peptides.

The common cationicity of AMPs possibly creates a stronger preference for the anionic bacterial membranes. Furthermore, AMPs frequently feature hydrophobic stretches. The structure of most AMPs shows roughly $50 \%$ hydrophobicity, which is an important feature that enables the peptides to insert into the hydrophobic core of the membrane [16]. When interacting with the target membrane, AMPs may change their conformation to segregate polar and apolar peptide regions, creating amphiphaticity to allow insertion into the lipid bilayer [17]. 


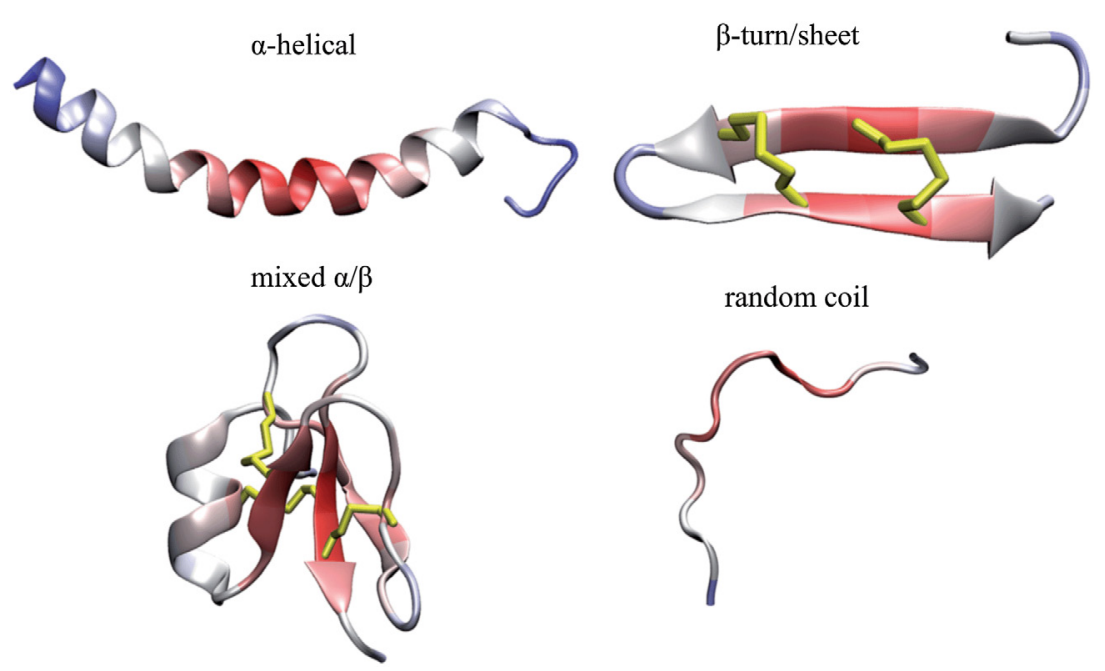

Fig. 1.1: Overview of characteristic secondary structural motives of AMPs. Peptides can be grouped into the major classes $\alpha$-helical, $\beta$-sheet, a mix of the secondary structures, and other- here represented by random coil. Image source: [1]

$\boldsymbol{\alpha}$-helical peptides make up the largest group of antimicrobial peptides (Fig. 1.1 top left). The most well-known examples are cecropins and magainins, cathelicidins and melittin [18][19][20][21]. A magainin as well as melittin are also investigated in this work. The structure of the peptides may form upon binding to the membrane. The helix of these peptides can be kinked or interrupted by a flexible, unordered linker [21].

$\boldsymbol{\beta}$-sheet peptides are another group of antimicrobial peptides whose molecular structure is mainly characterized by $\beta$-strands and only few $\alpha$-helical structural elements (Fig. 1.1 top right, bottom left). A few well-known examples are gramicidines. Here, there are also variations in cyclic forms known, such as gramicidin S [22].

A large group of antimicrobial peptides does not fit into the aforementioned classes. They may not exhibit particular structural motives (Fig. 1.1 bottom right), but may share similarities by featuring regions rich in certain amino acids such as increased amounts of tryptophan [23], proline and arginine, or histidines [24]. These amino acids impose structural constraints on the peptide structure [7]. While these structures differ from helices or sheets; these AMPs may adopt characteristic conformations in the membrane. Examples are the tryptophan-rich indolicidin, which appears to adopt a wedge shape once in the membrane [25], or the proline/ arginine rich PR-39, which forms a polyproline helix [26].

\subsubsection{AMP modes of action on the membrane}

The primary target of AMPs is thought to be the membrane of pathogens [6], which is perturbed and possibly permeabilized by these peptides. Permeabilization of the membrane leads to a dissipation of the electrochemical potential, the asymmetry of membrane lipids, 
leaking of metabolites and cellular components. As a consequence, the affected cell shrinks and may ultimately die [7].

Additionally, AMPs were shown to be signal mediators and immunomodulators of the innate as well as the adaptive immune response [27][21]. Some show antiviral activity [28][29], act as templates for peptides that penetrate cells [30], or are discussed to have antitumoral effects [12]. They are considered to have the potential to interfere with core functions of the metabolism. This work will focus on the first mentioned and best-studied feature of the AMPs, their membrane perturbing characteristics.

The specificity of AMPs towards pathogen membranes as opposed to the membranes of their host is achieved by preference for the lipid composition, charge, and electronegativity of the pathogen [31][32][33]. There are thought to be threshold concentrations to AMP action [34], which are independent from the subsequent mechanism of action of the peptide [17][35].

A variety of different modes of action is depicted in Fig. 1.2. The following paragraphs explore a few modes relevant for this work.

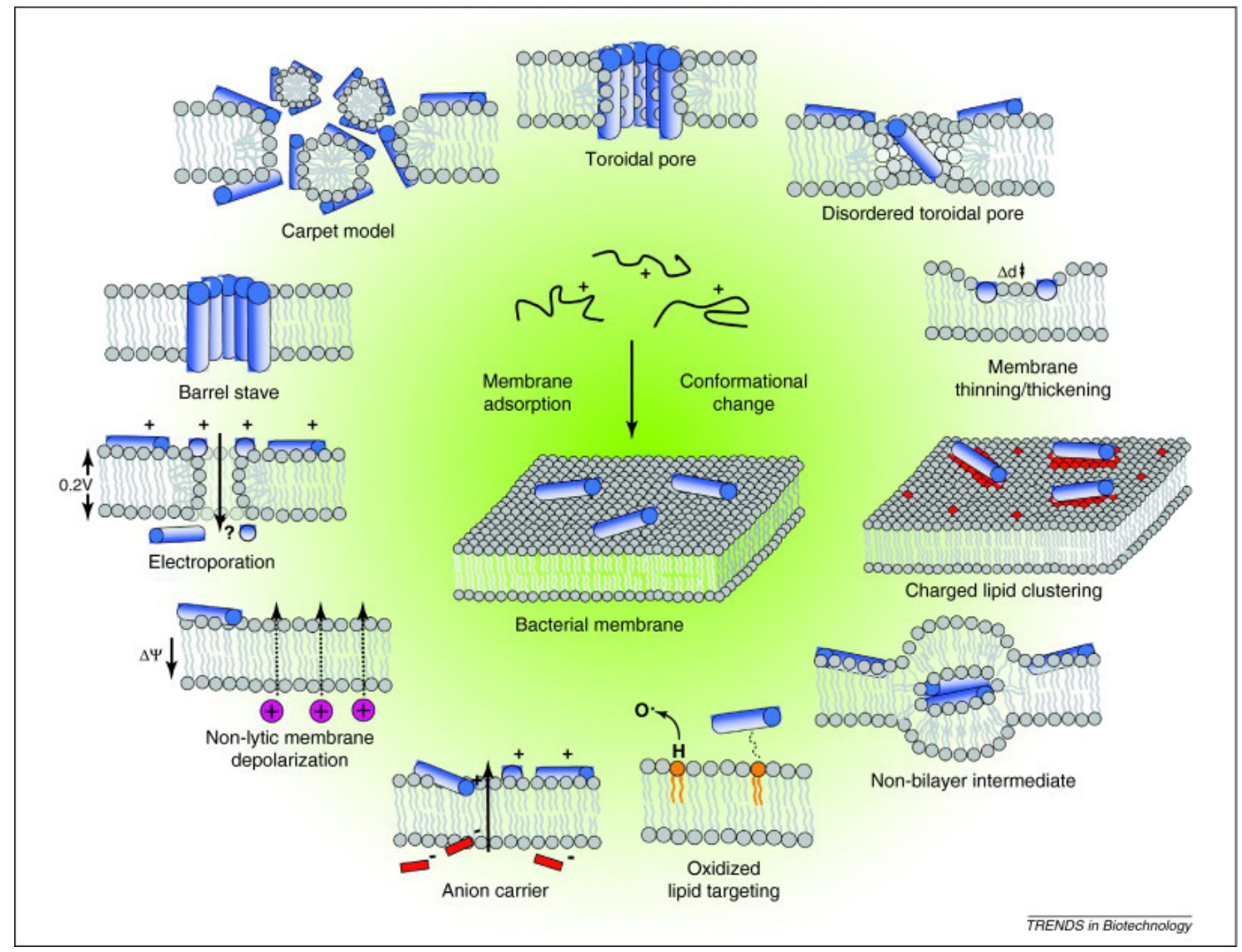

Fig. 1.2: Modes of interaction of antimicrobial peptides on the lipid bilayer. Image center shows the initial binding of the peptides to the lipid bilayer membrane. The surrounding illustrations depict possible subsequent changes of bilayer structure and characteristics by the antimicrobial peptides. Image source: [2]

The initial binding of the cationic antimicrobial peptide to the pathogen membrane occurs via 
electrostatic interactions with the negatively charged phospholipid head groups of certain membrane lipids. It may induce the formation of the peptide's secondary structure [21] (Fig. 1.2 middle). Upon reaching the threshold concentration of adhered AMPs, one or a combination of certain modes of action leads to membrane perturbation.

The carpet model describes a mode of action where peptides adhere parallelly to the surface of the lipid bilayer [36]. Lysis is induced in a detergent-like manner [37]. This allows additional peptides to bind, from which the membrane disintegrates [7]. Insertion into the membrane is thought to be thermodynamically driven [38][39].

In the barrel-stave model, the amphiphatic peptides bind to the lipid membrane (possibly as monomers). Subsequently, those bound peptides oligomerize, allowing the hydrophobic portion of the peptides to insert into the membrane [40]. This way, a highly ordered transmembrane pore is formed with the peptides oriented perpendicularly to the bilayer. The hydrophobic peptide portion faces the hydrophobic membrane core, while the hydrophilic part lines the newly formed pore center [41].

The toroidal pore model also advocates the formation of pores through the perpendicular insertion of peptides into the lipid bilayer. In contrast to the barrel stave model, the AMPs locally induce curvature on the lipid monolayers. The hydrophilic regions of the peptides make contact both with the head groups of the lipids, as well as with the hydrophilic regions of other inserted peptides, forming a pore from peptides as well as polar head groups of lipids in the center of the pore. This also includes the creation of a bridge between inner and outer leaflet of the bilayer [42][7].

The disordered toroidal pore model is a variant where less rigid peptide orientations (other than perpendicularity) in the membrane are proposed [36] [40] [43].

During membrane thinning, lipid material is removed from the membrane, destabilizing the membrane und ultimately leading to rupture. This may occur only on one side of the lipid bilayer [44][45]-[47][48].

Several other modes of action have been proposed and shall be mentioned briefly here. Other perturbations of membrane structure and integrity are membrane thickening and the formation of non-bilayer membrane intermediates. Electrochemical properties of the cell membrane are targeted by the mechanisms of charged lipid clustering up to lateral lipid segregation that may rearrange the membrane components with effects on membrane stability and functionality [49]. Molecular electroporation leads to the dissipation of the electrochemical potential, and anion carrier activity transfers small charged molecules across the membrane, resulting in loss of intracellular material and non-lytic membrane depolarization [21][40][17]. An opposing hypothesis discusses that there is a simple lytic mechanism underlying AMP action, which leads to lysis of the membrane when the AMP concentration is high enough [17]. 
Indeed it is thought that the modes of action of most AMPs do not originate from exclusive mechanisms, but are a combination of different effects with a continuous graduation between them, possibly in dependence on AMP concentration. This is reflected in the observation that the action of AMPs often impairs but does not destroy electrochemical, ion, and $\mathrm{pH}$ gradients [21].

\subsubsection{Peptides used in this work}

\subsubsection{Magainin-2}

Magainin-2 is one of the magainin family proteins found in the African clawed frog Xenopus laevis [50]. It is 23 amino acids long and has the sequence GIGKFLHSAKKFGKAFVGEIMNS. The peptide adopts an $\alpha$-helical structure (Fig. 1.3) upon binding to the membrane [51][52][53] and was reported to form amyloid-like structures in the presence of acidic phospholipids [54][55]. These structures appear to be important for its activity.

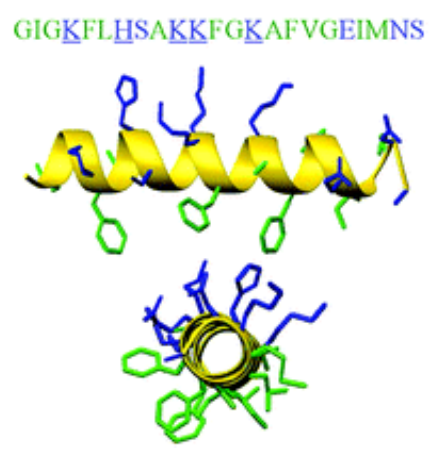

Fig. 1.3: Magainin-2 secondary structure as determined by NMR, based on PDB entry 2MAG. The peptide adopts an $\alpha$-helical fold upon interaction with the lipid membrane (Two-dimensional 1H NMR experiments show that the 23-residue magainin antibiotic peptide is an alpha-helix in dodecylphosphocholine micelles, sodium dodecylsulfate micelles, and trifluoroethanol/water solution [1]). Image source [3].

Magainins were reported to unfold their antimicrobial action through toroidal pore formation [56]. Magainin-2 shows a broad range of antibacterial, antifungal, and antitumor effects.

\subsubsection{Melittin}

Melittin is an antimicrobial peptide produced by the honey bee Apis mellifera. It was first reported 1982 by Terwilliger et al. [57]. It is 26 amino acids long, with the sequence GIGAVLKVLTTGLPALISWIKRKRQQ. Melittin belongs to the class of $\alpha$-helical peptides (Fig. 1.4), adopting a structure of two $\alpha$-helical regions that are separated by a non-helical segment in the middle [58][59]. The N-terminus of the peptide is hydrophobic, while the Cterminus is basic [57]. Like magainin-2, melittin was reported to form protofibrilliar structures on the target membrane [54][55]. 


\section{$\operatorname{Lin} \cos :$}

Fig. 1.4: Melittin structure as determined by NMR, based on PDB entry 2MLT. Two $\alpha$-helical domains are connected by a linker.

Melittin possesses a broad range of antibacterial activity and is also discussed to act antivirally [18]. A cationic cecropin-melittin hybrid peptide was shown to significantly reduce inflammatory responses caused by endotoxins [60]. Melittin has been proposed to act on lipid bilayers by toroidal pore formation [61] or carpet formation [40][36][62] .

\subsection{Model membrane systems for the characterization of AMPs}

Besides in vivo assays, simplified model lipid bilayers like vesicles with defined composition are used to investigate AMPs. Since all model systems are limited in some aspects, it is often mandatory to combine model systems to fully address aspects of membrane interaction.

In order to test which AMPs follow which mode of action, model membrane systems have proven a very helpful tool. They allow for the characterization of the modes of action of known AMPs as well as the identification of new antimicrobial substances. They mimic a complex system like a eukaryotic cell in a minimal way, typically encompassing just a lipid bilayer (or monolayer) and some sort of aqueous compartment(s). A few approaches are listed in the following chapters, with the focus on the system investigated in this work.

\subsubsection{Vesicles}

Unilammellar vesicles have been used to study membrane interactions of AMPs $[61][63][64][65]$. The advantage of the system is that the vesicle preparation is comparably easy and reproducible. The disadvantage is that these systems largely rely on bulk release assays, where the overall changes in the system are monitored. Because the vesicles float in solution, individual membranes cannot be monitored over time. To overcome this, vesicles may be linked to a substrate. Another issue is the membrane curvature of the vesicles. Curvature produces an increased tension in comparison to the less curved planar membranes of physiological target organisms of AMPs, which can lead to a bias towards membrane perturbations or rupture.

\subsubsection{Solid supported bilayers}

Solid supported bilayers rely on the application of a lipid bilayer onto a substrate with an aqueous supernatant. Various techniques to generate solid supported membranes can be found in [66]. The lipid membrane retains its fluidity through a thin 10 to 20 Angstrom thick layer of water that is trapped between membrane and support [67][68]. The membranes on these 
supports are stable and can be probed with techniques that analyze the surface of the membrane. A disadvantage is that they do not have a second aqueous compartment underneath the lipid layer in the way that cells do, limiting the scope of experiments to surface analyses. Furthermore, lipids only have a reduced ability to move laterally in these membranes [69] [70]. This has been addressed by introducing spacers between the solid support and the lipid bilayer such as flexible polymer networks [71].

\subsubsection{Black lipid membranes}

Black lipid membranes were first described by Muller et al., and they carry their name because of their appearance under optical microscopy [72][73]. They consist of phospholipid molecules that span an aperture between two solution chambers with a diameter of less than $1 \mathrm{~mm}$. Advantages of black lipid membranes over solid supported bilayers are for example that they avoid direct contact with the underlying substrate, as this could cause problems for integrated transmembrane proteins. They also allow solution phase access to both sides of the membrane. However, they are less stable than solid supported membranes, their chemical manipulation is more difficult, and they are much less accessible to surface specific detection techniques [74][75].

\subsubsection{Pore-spanning membranes}

Pore-spanning membranes are lipid bilayers that are stretched over a porous support matrix. The pore rims provide support for the lipid bilayer with aqueous compartments on each membrane side, making them accessible from both sides [76]. In contrast to vesicles, the porespanning membranes do not have a strong curvature, which makes them an interesting model system to mimic a cell membrane. Unlike in solid supported membranes, membrane fluidity is maintained in pore-spanning membranes [77].

The membranes are spatially fixed on the substrate. This provides possibilities for optical readout methods, enabling the monitoring of individual membrane patches over time. Because of this, pore-spanning membranes may allow for simultaneous investigation of changes of the membrane structure as well as translocation events from one aqueous compartment to the other.

There are several approaches to prepare pore-spanning membranes: Different matrix materials and porous substrate preparation procedures lead to differently sized pores. The electrostatic properties of the substrate surface may call for functionalization before membrane preparation, and the membranes can be composed with different lipid ratios to reflect the composition of different biological membranes [78]. Membrane application onto the substrate has been accomplished by spreading unilamellar vesicles [79][80]-[82] or membrane painting $[83][84]-[86]$. 
Solid-supported membrane systems are also compatible with various readout approaches. By doping membranes with fluorescently labeled lipids it is possible to monitor changes in membrane structure and topology by fluorescence microscopy. The addition of a fluorescent dye to an aqueous compartment allows for the detection of membrane permeabilization events. This may either be done by monitoring the release of a dye from pores, resulting in fluorescence signal reduction, or by measuring dye entry through fluorescence increase. Other readout methods include, but are not limited to, atomic force microscopy, impedance spectroscopy, or ion conductance microscopy. 


\subsection{Aim of this work}

In this work, pore-spanning membranes are investigated for their potential to monitor AMP activity, using fluorescence-based assays. For this, the preparation of different porous support materials is established, the materials are characterized and evaluated for their versatility in permeabilization assays: Membranes derived from GUV spreading onto the support are characterized and probed with antimicrobial peptides (AMPs). The effects of the AMPs magainin-2 and melittin on artificial membranes are evaluated in detail. Based on these data, conclusions on their mode of action are drawn.

The new information obtained on well-characterized AMPs can be used to test potential peptide antibiotics. This lays the groundwork for clinical applications and research applications where cell membranes need to be passed, but also for any scientific studies focusing on the investigation of membrane-associated processes. 


\section{Materials and Methods}

\subsection{Materials}

\subsubsection{Technical Equipment}

Fluorescence microscopy

Olympus BX51 Olympus (Tokyo, Japan)

LSM 710 Examiner Carl Zeiss GmbH (Jena)

\section{Confocal laser scanning microscopy (CLSM)}

LSM 710 Examiner

Carl Zeiss, (Jena)

objective:

Carl Zeiss, (Jena)

WPlan APOChromat 63_1.0

ENTH-Alexa488 $\lambda_{\mathrm{ex}}=488 \mathrm{~nm}, \lambda_{\mathrm{em}}=499-567 \mathrm{~nm}$

Texas Red DHPE $\lambda_{\mathrm{ex}}=561 \mathrm{~nm}, \lambda_{\mathrm{em}}=569-699 \mathrm{~nm}$

Fluorescence spectroscopy

JASCO FP 6500

JASCO (Easton, USA)

UV/vis spectroscopy

Varian Cary Scan 50

Varian (Darmstadt)

Quartz cuvette

Hellma (Mühlheim)

\section{Surface coating}

MED 020 Modular High Vacuum

Bal-Tec AG (Balzers, Liechtenstein)

Sputter Coater 108auto

Cressington (Watfort, UK)

Thickness Controller MTM20

Cressington (Watfort, UK)

\section{Miscellaneous equipment}

Water purifier MilliQ Gradient A10

Millipore (Eschborn)

Precision scale CP225D

Sartorius (Göttingen)

pH-meter Calimatic 766

Knick (Berlin) 
Ultrasonic bath Sonorex RK $255 \mathrm{H}$

Plasma Cleaner PDC 32 G-2

Frequency generator Agilent 3220A

Extruder

Osmometer Osmomat 030/3000

\subsubsection{Software}

ZEN 2012

ImageJ $1.45 \mathrm{~s}$

Matlab R2012b

Graphics Layout Engine
Badelin (Berlin)

Harrick (New York City, USA)

Agilent Technologies (Santa Clara, USA)

Avestin (Ottawa, Canada)

Gonotec (Berlin)

http://rsbweb.nih.gov/ij

MathWorks (Natick, USA)

http://www.gle-graphics.org/

\subsubsection{Chemicals}

Aqueous buffer solutions were prepared with ultrapure water $(R>18.2 \mathrm{M} \Omega)$ from, a Milli-Q Gradient A10 (Millipore, Eschborn, Germany).

Alexa Fluor 488 carboxylic acid

Aluminium $99.999 \%$

Ammonia solution (25\%)

Argon

Cholesterol

Chloroform

Copper tape

DOPC

DOPE

Disodium hydrogen phosphate

$\mathrm{DPhPC}$

Ethanol, p.a.

Carbodiimide glass slides D $263 \mathrm{M}$

Glucose

Glycerin
Invitrogen (Eugene, USA)

Goodfellow, Bad Nauheim

VWR International (Darmstadt)

Air Liquide Deutschland GmbH (Düsseldorf)

Sigma-Aldrich (Taufkirchen)

VWR International (Darmstadt)

Präzisionsglas \& Optik GmbH (Iserlohn)

Avanti Polar Lipids (Alabaster, USA)

Avanti Polar Lipids (Alabaster, USA)

Merck KGaA (Darmstadt)

Avanti Polar Lipids (Alabaster, USA)

VWR International (Darmstadt)

SCHOTT (München)

Carl Roth GmbH (Karlsruhe)

Honeywell Riedel de Häen AG (Seelze) 
Gold (99.99 \%)

Hydrochloric acid

Hydrogen peroxide solution (30 \%)

ITO slides

Mercaptoethanol

Mercaptohexanol

Methanol

Mucasol $^{\circledR}$

$n$-propanol

Parafilm $^{\circledR}$

POPC

POPE

Polycarbonate membranes

Potassium chloride

Potassium hydroxide

Porous Si substrates, closed

Porous Si substrates, open

$n$-propanol

Oxygen

Sodium chloride

Sodium citrate

Sodium dodecyl sulfate

Sucrose

Texas Red DHPE

Titan Target

TRIS

Petri dishes

Pyranine
Allgemeine Gold- und Silberscheideanstalt (Pforzheim)

Merck (Darmstadt)

Carl Roth GmbH (Karlsruhe)

Präzisions Glas \& Optik GmbH (Iserlohn)

Sigma-Aldrich (Taufkirchen)

Sigma-Aldrich (Taufkirchen)

Carl Roth GmbH (Karlsruhe)

Merck (Darmstadt)

Carl Roth GmbH (Karlsruhe)

Pechiney Plastic Packaging (Chicago, USA)

Avanti Polar Lipids (Alabaster, USA)

Avanti Polar Lipids (Alabaster, USA)

Avestin (Ottawa, Canada)

Carl Roth GmbH (Karlsruhe)

Merck (Darmstadt)

Dr. Siegfried Steltenkamp, CEASAR (Bonn)

Fluxxion (Eindhofen, Netherlands)

Merck (Darmstadt)

Air Liquide Deutschland GmbH (Düsseldorf)

Merck (Darmstadt)

Merck (Darmstadt

AppliChem GmbH (Darmstadt)

Carl Roth GmbH (Karlsruhe)

Sigma-Aldrich (Taufkirchen)

Elektronen Optik Service GmbH (Dortmund)

Carl Roth GmbH (Karlsruhe)

VWR International GmbH (Nürnberg)

Acros Organics (New Jersey, USA) 


\subsubsection{Lipids and detergents}

Synthetic phospholipid, sterol and detergent solutions were prepared in $\mathrm{CHCl}_{3}$.

\subsubsection{POPC}

POPC (1-Palmitoyl-2-oleoyl-sn-glycero-3-phosphocholine, $\mathrm{C}_{42} \mathrm{H}_{82} \mathrm{NO}_{8} \mathrm{P}$ ) is a phospholipid with a molecular weight of $760.1 \mathrm{~g} \cdot \mathrm{mol}^{-1}$. It was obtained from Avanti Polar Lipids, Alabaster (USA) and stored at $-20^{\circ} \mathrm{C}$.

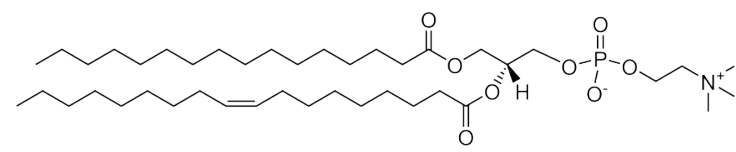

Fig. 2.1: Structure of 1-palmitoyl-2-oleoyl-sn-glycero-3-phosphocholine (POPC).

\subsubsection{Sphingomyelin (SM)}

Sphingomyelin (SM, main component $\mathrm{C} 18: 0, \mathrm{C}_{41} \mathrm{H}_{83} \mathrm{~N}_{2} \mathrm{O}_{6} \mathrm{P}$ ) is a sphingolipid with a molecular weight of $731.1 \mathrm{~g} \cdot \mathrm{mol}^{-1}$. It was obtained from Sigma-Aldrich (Taufkirchen) and stored at $-20{ }^{\circ} \mathrm{C}$.

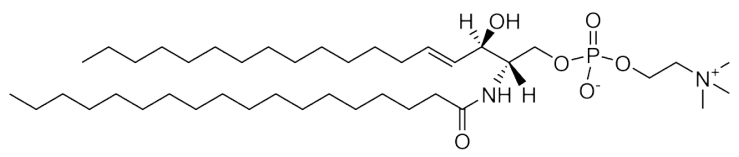

Fig. 2.2: Octadecanoylsphingomyelin (SM, C18:0 Brain, Porcine), structure of predominant species.

\subsubsection{DOPE}

DOPC (1,2-di-(9Z-octadecenoyl)-sn-glycero-3-phosphoethanolamine, $\left.\mathrm{C}_{44} \mathrm{H}_{84} \mathrm{NO}_{8} \mathrm{P}\right)$ is a phospholipid with a molecular weight of $786.1 \mathrm{~g} \cdot \mathrm{mol}^{-1}$. It was obtained from Avanti Polar Lipid (Alabaster, USA) and stored at $-20{ }^{\circ} \mathrm{C}$.

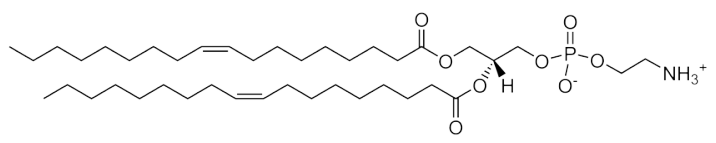

Fig. 2.3: Structure of 1,2-di-(9Z-octadecenoyl)-sn-glycero-3-phosphoethanolamine (DOPE).

\subsubsection{Cholesterol}

Cholesterol (Chol, (3ß)-Cholest-5-en-3-ol, $\mathrm{C}_{27} \mathrm{H}_{46} \mathrm{O}$ ) is a sterol with a molecular weight of $386.65 \mathrm{~g} \cdot \mathrm{mol}^{-1}$. It was obtained from Sigma-Aldrich (Taufkirchen), and stored at $-20{ }^{\circ} \mathrm{C}$.

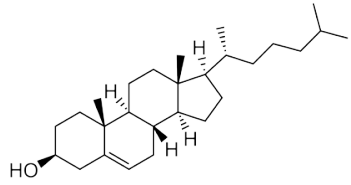

Fig. 2.4: Structure of Cholesterol 


\subsubsection{Triton $\mathrm{X}-100$}

Triton X-100 (polyethylene glycol p-(1,1,3,3-tetramethylbutyl)-phenyl ether (Merck KGaA, Darmstadt) is a non-ionic detergent with a molecular weight of $647 \mathrm{~g} \cdot \mathrm{mol}^{-1}$ that solubilizes lipid membranes.

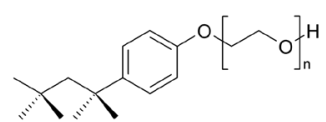

Fig. 2.5: Structure of Triton X-100 (polyethylene glycol p-(1,1,3,3-tetramethylbutyl)-phenyl ether), $n=$ 100 .

\subsubsection{Fluorophores}

\subsubsection{TexasRed DHPE}

Texas Red DHPE (Sulforhodamine 101 1,2-dihexadecanoyl-sn-glycero-3-phosphoethanol-1amine triethylammonium salt, Santa Cruz Biotechnology Inc., Heidelberg) is a lipid-anchored fluorescent dye $\left(\lambda_{e x} 584 \mathrm{~nm} ; \lambda_{e m} 608 \mathrm{~nm}\right.$ ) with a molecular weight of $1381.84 \mathrm{~g} \cdot \mathrm{mol}^{-1}$.

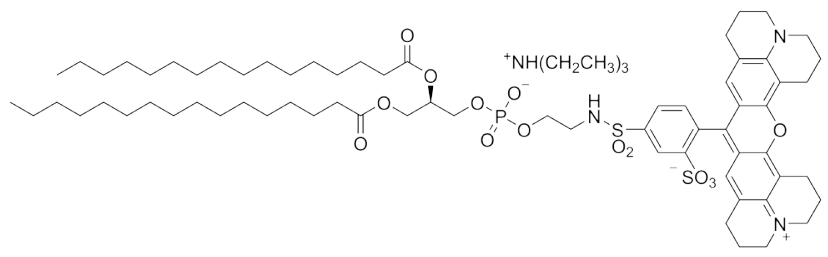

Fig. 2.6: Structure of Sulforhodamine 101 1,2-dihexadecanoyl-sn-glycero-3-phosphoethanol-l-amine triethylammonium salt (Texas Red DHPE)

\subsubsection{FITC Dextran (70 kDa, 40 kDa, 10 kDa)}

Fluorescein isothiocyanate-dextran (FITC-dextran, Sigma-Aldrich Taufkirchen) is a water soluble fluorescent dye $\left(\lambda_{e x} 492 \mathrm{~nm} ; \lambda_{e m} 518 \mathrm{~nm}\right)$. Dextrans are polysaccharides composed of glucose subunits that feature defined hydrodynamic (Stokes-) radii. The labeling rate of the dextrans is 1:250 (FITC:Glucose). The Stokes radii are as follows:

FITC dextran with $70 \mathrm{kDa}(\operatorname{Dex} 70)$ : approx. $6.0 \mathrm{~nm}$;

FITC dextran with $40 \mathrm{kDa}(\mathrm{Dex} 40)$ : approx. $4.5 \mathrm{~nm}$;

FITC dextran with $10 \mathrm{kDa}$ (Dex10): approx. $2.3 \mathrm{~nm}$.

\subsubsection{Pyranine}

Pyranine (Trisodium 8-hydroxypyrene-1,3,6-trisulfonate, Acros Organics b.v.b.a., Geel, Belgium) is a water-soluble fluorescent dye with a molecular weight of $524.39 \cdot \mathrm{rgol}{ }^{-1}$. Pyranine is $\mathrm{pH}$-sensitive with $\lambda_{e x} 492 \mathrm{~nm} ; \lambda_{e m} 518 \mathrm{~nm}$ at $\mathrm{pH} 7.4$. 


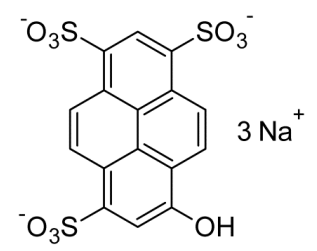

Fig. 2.7: Structure of trisodium 8-hydroxypyrene-1,3,6-trisulfonate (Pyranine).

\subsubsection{Antimicrobial peptides (AMPs)}

\subsubsection{Melittin}

Melittin is known as a cell membrane lytic factor (see 1.1.3.2). At physiological pH 7.4 it is highly cationic (charge +5 to $+6, \mathrm{pI}=12.02$ [87]. The purchased melittin compound (Sigma Aldrich, Saint Louis, MO, USA) was isolated from bee venom with a puxit $\$ 586$ (HPLC).

The concentration of the used stock solution was determined by UV/Vis spectroscopy at a wavelength of $\lambda=280 \mathrm{~nm}$ with an extinction coefficient of $\varepsilon=5500 \mathrm{~cm}^{-1} \mathrm{M}^{-1}$ [87]. The stock solution was aliquoted, frozen in liquid nitrogen and stored at $-20{ }^{\circ} \mathrm{C}$.

\subsubsection{Magainin-2}

Magainins are a class of antimicrobial peptides that were isolated from the African clawed frog (Xenopus laevis) [50]. Magainin-2 is a peptide consisting of 23 amino acids and a molecular mass of $2.467 \mathrm{kDa}$. At physiological $\mathrm{pH} 7.4$ the net charge is +4 [88]. The synthesized magainin-2 compound (LKT Laboratories, Inc., St. Paul, MN, USA) was purchased with a purity of $\geq 95 \%$. The peptide was dissolved in $\mathrm{H}_{2} \mathrm{O}$, aliquoted and stored at $20{ }^{\circ} \mathrm{C}$.

\subsubsection{3 $\delta$-lysin}

The peptide $\delta$-lysin is a 26-residue hemolytic peptide secreted by Staphylococcus aureus with a molecular mass of $2.979 \mathrm{kDa}$. The peptide sequence is MAQDIISTIGDLVKWIIDTVNKFTKK. $\delta$-lysin is neutral at physiological $\mathrm{pH}$ of 7.4. It is lytic by solubilizing cell membranes.

The synthesized $\delta$-lysin compound (AnaSpec, Fremont, CA, USA) was purchased in a purity of $\geq 95 \%$. The peptide was dissolved in $\mathrm{H}_{2} \mathrm{O}$, aliquoted and stored at $-20{ }^{\circ} \mathrm{C}$.

\subsection{Preparative Methods}

All methods are described in principle at first, followed by a more detailed explanation including the precise parameters. 


\subsubsection{Giant unilamellar vesicle (GUV) preparation}

In this thesis, giant unilamellar vesicles (GUVs) were prepared through controlled rehydration of deposited lipid films on a solid surface. The rehydration is controlled by the application of an external AC electric field (electroformation) as described by [89][90].

The lipids dissolved in a suitable organic solvent were deposited on a conducting medium (indium tin oxide (ITO) coated glass slides, Präzisionsglas \& Optik GmbH, Iserlohn, Germany) and dried under reduced pressure. Upon drying, the lipids arrange in multiple parallel layers of lipid bilayers and are therefore already pre-organized. In the next step, the lipid films were rehydrated by the addition of water or an aqueous sugar solution. Water addition first leads to hydration of the hydrophilic head groups and then the water continuously diffuses into the space in between the bilayers. After rehydration, the top lipid layers can reorganize to form giant unilamellar vesicles. Simultaneous release of underlying lipid bilayers can lead to the formation of multilamellar vesicles. Application of an electric field led to light mechanical movement of the lipid layers und supported the release of unilamellar vesicles. After electroformation the resulting unilamellar GUV solution were removed from the ITO chamber and stored at room temperature in the dark for a few days.

\subsubsection{Preparation of POPC/Texas Red GUVs}

POPC and Texas Red DHPE were dissolved in chloroform. POPC (99.5 mol\%) and Texas Red DHPE $(0.5 \mathrm{~mol} \%)$ were mixed in a vial to a total lipid content of $0.1 \mathrm{mg}$ and kept cool and dark.

The electroformation chamber was prepared of two ITO slides that sealed a Teflon ring in which the buffer solution was added. The ITO slides were separated by two Teflon spacers on which adhesive copper tape was placed to connect the electrodes, and fixed in position using binder clips. A cut in the Teflon ring allowed opening and closing of the ring to add buffer solution.

In a first step, the lipids were deposited onto the ITO slides in the areas to be sealed by the Teflon ring and dried under reduced pressure for at least $15 \mathrm{~min}$ at room temperature to remove the chloroform. Subsequently the Teflon ring was placed around the dried lipid droplet of one ITO slide and a second ITO slide was positioned above to seal the chamber.

Sucrose solution $(300 \mathrm{mM}, 1.5 \mathrm{~mL})$ was added to the chamber and the electrodes were connected with a frequency generator (33220 A, Agilent Technologies, Böblingen). A sinusoidal alternating current (AC) with a voltage of $3 \mathrm{~V}$ and $5 \mathrm{~Hz}$ was applied for at least $3 \mathrm{~h}$. Afterwards the vesicle solution was harvested and stored in an Eppendorff tube and stored in the dark at room temperature. 


\subsubsection{Preparation of phase-separated GUVs}

The ternary lipid mixture of sphingomyelin (SM), DOPC and cholesterol (Chol.) was used to generate GUVs that showed a separation of liquid-disordered $\left(l_{d}\right)$ and sphingomyelinenriched, liquid-ordered $\left(l_{0}\right)$ phase at room temperature.

The deposition and rehydration had to be carried out in the liquid-disordered state at a temperature above the melting temperature $T_{m}$. The electroformation was done using a commercial setup (Vesicle Prep Pro, Nanion) that allowed heating of the ITO slides.

The lipids were mixed and doped with Texas Red DHPE (40 mol\% SM/39.5 mol\% DOPC/ $20 \mathrm{~mol} \%$ Chol. $/ 0.5 \mathrm{~mol} \%$ Texas Red) to a final amount of $0.1 \mathrm{mg}$ total lipid.

The mixture was added to the conductive side of an ITO slide. Both ITO slides were placed into a vacuum drying oven at $55^{\circ} \mathrm{C}$ for at least $3 \mathrm{~h}$ or overnight. The ITO slides were mounted to the Vesicle Prep Pro and after addition of $320 \mu \mathrm{L}$ sucrose solution $(300 \mathrm{mM})$ the chamber was assembled and connected. The electroformation was run with the subsequent parameters.

The ITO slides were preheated to $55^{\circ} \mathrm{C}$ for $50 \mathrm{~min}$. An AC current $(1.6 \mathrm{~V}, 12 \mathrm{~Hz}$ ) was applied for $180 \mathrm{~min}$, then the frequency was reduced $(1.6 \mathrm{~V}, 5 \mathrm{~Hz})$ for $20 \mathrm{~min}$. Subsequently, the amplitude and frequency were gradually reduced to $0 \mathrm{~V}$ and $0 \mathrm{~Hz}$ over $15 \mathrm{~min}$ and the chamber was cooled to $20{ }^{\circ} \mathrm{C}$. Finally, the vesicle solution was harvested and stored in an Eppendorf tube in the dark and at room temperature.

\subsubsection{Preparation of nanoporous anodic aluminium oxide (AA0)}

In this thesis, porous and optically transparent anodic aluminium oxide (AAO) was used as a support for pore-spanning membranes. The AAO itself was adhered on a glass chip to improve stability and to allow easy mounting to a measuring chamber.

The pores of the AAO are highly ordered and arranged in a hexagonal, honeycomb-like pattern. The pores have a cylindrical shape with a depth of $7-10 \mu \mathrm{m}$. They are closed by a round shaped bottom and underlying aluminium oxide layer. The pore diameter is $65-75 \mathrm{~nm}$ and the lattice constant, the distance between the pore centers, is $\sim 100 \mathrm{~nm}$ (see Fig. 2.8). 


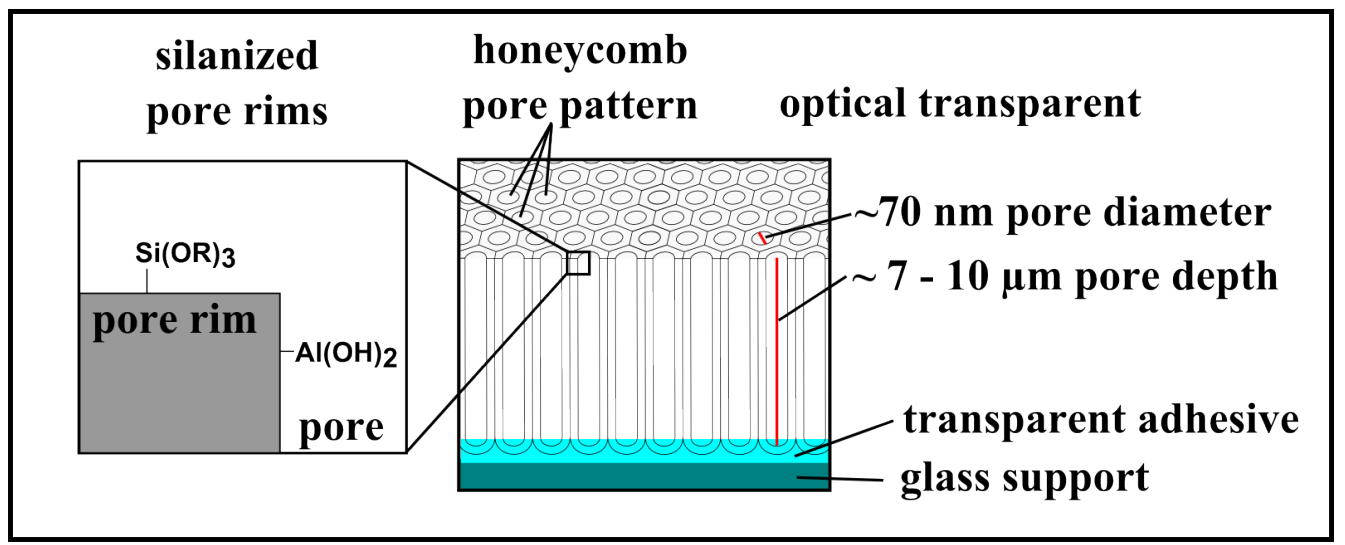

Fig. 2.8: Schematic representation of the AAO pore arrangement.

The AAO features optical transparency due to the small pore spacing, giving it optical properties which are below the diffraction limit of visible light.

Since the adhesive forces are very high on unfunctionalized alumina, vesicle spreading does hardly lead to lipid bilayer formation [91]. Therefore, the surface was hydrophilically functionalized by silanization with (3-mercaptopropyl)triethoxysilane (ABCR, Karlsruhe, Germany).

\subsubsection{Anodic oxidation}

Anodic oxidation is a very commonly used and important industrial process. Anodic aluminium oxide (AAO) was prepared by oxidizing an aluminium template in an electrochemical cell where the aluminium serves as the anode. In general, the Al template is placed in an electrolyte bath and a DC voltage is applied between the Al template (positive terminal; anode) and a cathode (negative terminal). The overall reaction is a redox reaction, in which the $\mathrm{Al}$ is oxidized to $\mathrm{Al}_{2} \mathrm{O}_{3}$ in an aqueous electrolyte solution and the electrolyte itself is reduced at the cathode as such that:
At anode:
$2 \mathrm{Al}+9 \mathrm{H}_{2} \mathrm{O} \rightarrow \mathrm{Al}_{2} \mathrm{O}_{3}+6 \mathrm{H}_{3} \mathrm{O}^{+}+6 \mathrm{e}^{-}$
Eq. 1

At cathode:

$$
6 \mathrm{H}_{3} \mathrm{O}^{+}+6 \mathrm{e}^{-} \rightarrow 3 \mathrm{H}_{2}+6 \mathrm{H}_{2} \mathrm{O}
$$

Overall reaction

$$
2 \mathrm{Al}+3 \mathrm{H}_{2} \mathrm{O} \rightarrow \mathrm{Al}_{2} \mathrm{O}_{3}+3 \mathrm{H}_{2}
$$

Eq. 3

In industrial processes the focus is on passivating aluminium surfaces by increasing the thickness of the natural $\mathrm{Al}_{2} \mathrm{O}_{3}$ layer and increasing the surface roughness for better adhesion of paint particles or adhesives. The latter can be achieved by using acidic electrolytes, which leads to the formation of porous structures. In general, the surface structure of the AAO is determined by the choice of electrolyte, voltage, temperature, and oxidation time. 
Highly ordered porous aluminum oxide is prepared in a two-step process in which the aluminium is pre-textured by anodic oxidation of aluminium in oxalic acid and selective removal of the formed aluminium oxide layer. A second anodization under the same reaction conditions leads to highly ordered aluminium oxide.

An overview over the process and the used parameters is given in table 3.1, and the principle and the used parameters are described in the following chapters.

Anodic oxidized aluminium oxide (AAO) substrates were prepared from aluminium in a 3step process. In a first step, aluminium foils were annealed and polished, followed by a twostep anodization process to form highly-ordered aluminium oxide. Finally, the formed aluminium oxide layer was transferred to a glass support and the pores were widened to the desired pore diameter.

\subsubsection{Annealing and electrochemical polishing}

For the formation of highly ordered AAO it is mandatory to use aluminium with a surface roughness as low as possible and largest possible grain size. The latter can be achieved by annealing the aluminium at $500{ }^{\circ} \mathrm{C}$ for at least $3 \mathrm{~h}$.

At sufficiently high temperatures and when crystal recovery and recrystallization have fully taken place, a further reduction of the internal energy can only be achieved through a reduction of the grain boundaries. This is the driving force which leads to grain growth.

The surface roughness can be minimized by electrochemical polishing, leading to flat aluminium plates with a mirror-like surface.

The aluminium plates form the anode in an electrochemical cell with strong acids as an electrolyte and using a platinum wire as a cathode. Under a constant DC voltage, aluminium is oxidized and dissolved in the electrolyte and directed to the cathode where the reduction reaction occurs under hydrogen formation. Aluminium in protruded areas dissolves faster than in lowered areas and therefore the surface is leveled. 
Table 3.1: AAO preparation steps

Step

1) Annealing

2) Electrochemical polishing

3) $1^{\text {st }}$ anodization

4) $\mathrm{Al}_{2} \mathrm{O}_{3}$ removal

5) $2^{\text {nd }}$ anodization

6)Al dissolving
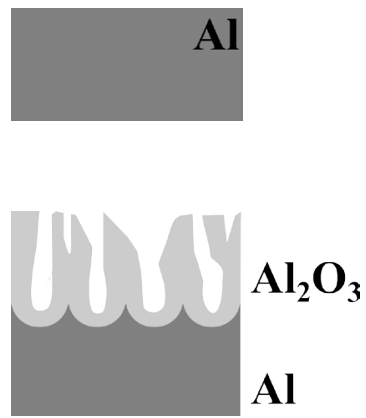

Al

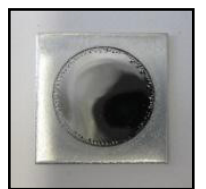

$\mathrm{H}_{2} \mathrm{SO}_{4} / \mathrm{H}_{3} \mathrm{PO}_{4} / \mathrm{H}_{2} \mathrm{O}(1: 1: 1)$; $25 \mathrm{~V} ; 65^{\circ} \mathrm{C} ; 2 \mathrm{x} 15 \mathrm{~min}$

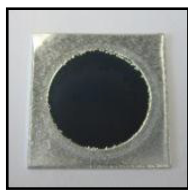

$0.3 \mathrm{M}$ oxalic acid

$40 \mathrm{~V} ; 1.5^{\circ} \mathrm{C} ; 2.5 \mathrm{~h}$

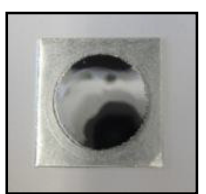

$5 \% \mathrm{H}_{3} \mathrm{PO}_{4}$

$\mathrm{rt} ; 2-3 \mathrm{~h}$;

$\mathrm{Al}_{2} \mathrm{O}_{3}$


$0.3 \mathrm{M}$ oxalic acid

$40 \mathrm{~V} ; 1.5^{\circ} \mathrm{C} ; 2.5 \mathrm{~h}$
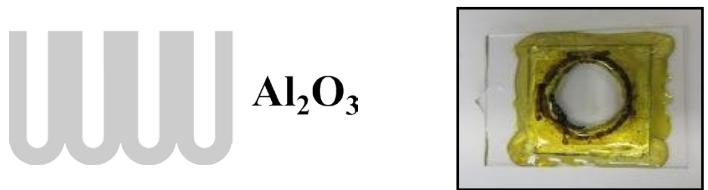

$17 \mathrm{~g} / \mathrm{L} \mathrm{CuCl}_{2}$

In $\mathrm{H}_{2} \mathrm{O} / \mathrm{HCl}(1: 1)$

$5 \% \mathrm{H}_{3} \mathrm{PO}_{4}$

7) Transfer

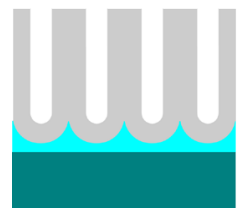

glass

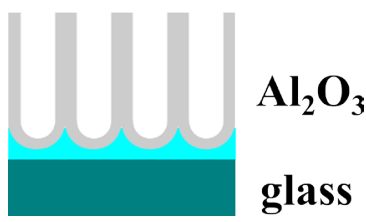

$20{ }^{\circ} \mathrm{C} ; 50 \mathrm{~min}$

$5 \% \mathrm{H}_{3} \mathrm{PO}_{4}$;

$20{ }^{\circ} \mathrm{C} ; 50 \mathrm{~min}$

gas phase silanization 
Used Parameters: Annealing and electrochemical polishing

Annealing (step 1)

Aluminium slides $\left(99.999 \%\right.$, Bad Nauheim) were annealed at $500{ }^{\circ} \mathrm{C}$ for at least $3 \mathrm{~h}$ under a nitrogen environment and square cut to fit the reaction chambers (edge length: $1.5 \mathrm{~cm}$, thickness: $0.5 \mathrm{~mm}$ ). The slides must be very flat, otherwise thin aluminium oxide layers can break very easily in the $\mathrm{Al}$ dissolving process.

\section{Electrochemical polishing (step 2)}

The aluminium was immersed in a chamber with the electrolyte and connected to the positive terminal of a DC voltage source while the cathode was connected to the negative pole.

Four aluminium plates were roughened on the backside with sandpaper and installed in a Teflon reaction chamber with copper base (anode). This polishing unit was placed on the preheated hot plate at $65{ }^{\circ} \mathrm{C}$ und filled with $25 \mathrm{~mL}$ of concentrated $\mathrm{H}_{2} \mathrm{SO}_{4} / 85 \% \mathrm{H}_{3} \mathrm{PO}_{4} / \mathrm{H}_{2} \mathrm{O}$ (1:1:1). A platinum wire mounted in the chamber cover was used as the cathode. After 5 min of equilibration a constant DC voltage of $25 \mathrm{~V}$ was applied for $15 \mathrm{~min}$. Then the electrolyte was exchanged and the procedure was repeated for $15 \mathrm{~min}$.

The plates were purged with $\mathrm{H}_{2} \mathrm{O}$ and p.a. ethanol. Any existing copper residues were removed with $\mathrm{HNO}_{3}$. Finally, the aluminium plates were incubated in $5 \% \mathrm{H}_{3} \mathrm{PO}_{4}$ for $1 \mathrm{~h}$.

\subsubsection{Two-step anodization of highly ordered anodic aluminium oxide (AAO)}

Anodic oxidation leads the formation of porous structures with hexagonal patterns on the metal surface [92]. In the 1990s, optimized two-step anodization procedures were developed leading to the formation of highly ordered pores arranged in a hexagonal pattern [93].

For anodization, the annealed and polished aluminium was placed in a chamber with oxalic acid as an electrolyte solution. A DC-voltage was applied between the Al template (positive terminal; anode) and a cathode (negative terminal).

The principle of the two-step anodization procedure is described in Fig. 2.9. 


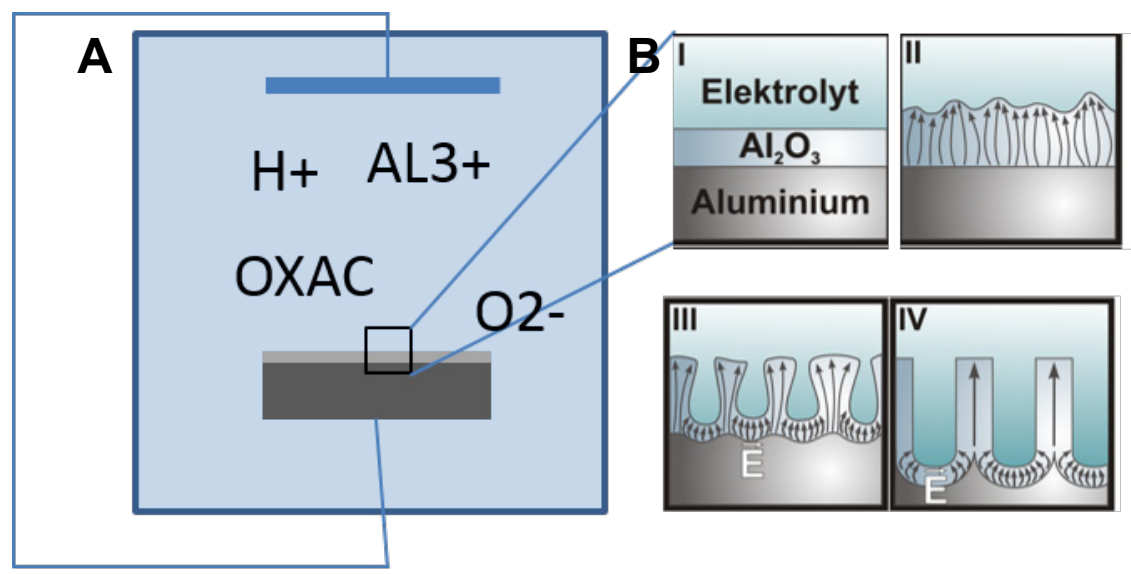

Fig. 2.9: Anodization of highly ordered anodic aluminium oxide. (A) Schematic representation of the field directed formation of aluminium oxide in an electrolyte (oxalic acid) filled chamber after application of a $\mathrm{DC}$ voltage. At the anode $\mathrm{Al}$ is oxidized to $\mathrm{Al}_{2} \mathrm{O}_{3}$, while positive ions migrate to the cathode and are reduced. The oxide formation is controlled by the field-aided transport of ions $\left(\mathrm{OH}^{-}, \mathrm{O}^{2-}\right.$ and $\left.\mathrm{O}^{\mathrm{x}-}\right)$ to the aluminium surface. (B) Schematic representation of an idealized pore formation process. (I) Initially the surface is covered with a compact barrier oxide layer. (II) Fluctuations of the surface morphology lead to distinct local field distributions. (III) Pore growth is initiated by the field enhanced $\mathrm{Al}^{3+}$ dissolution. (IV) Steady-state pore growth: Due to the high local field distribution in the pores, $\mathrm{Al}_{2} \mathrm{O}_{3}$ only grows at the sides. Volume expansion after oxidation pushes the formed pores upwards. Image Source: [2]

$\mathrm{Al}$ is oxidized and an initial $\mathrm{Al}_{2} \mathrm{O}_{3}$ barrier oxide is formed. The application of the $\mathrm{DC}$ voltage induces local electromagnetic fields in the $\mathrm{Al}_{2} \mathrm{O}_{3}$ which depends on the oxide thickness. $\mathrm{Al}^{3+}$ is exposed to a local field, moves to the $\mathrm{Al}_{2} \mathrm{O}_{3}$ surface, dissolves in the electrolyte and moves towards the cathode.

The process of pore formation is determined by an equilibrium between field directed alumina dissolution in the electrolyte and oxide formation on the aluminium surface.

The dissolution rate is dependent on the applied voltage and the resulting current density. The surface of the initially formed barrier oxide has a natural roughness. In areas of lower oxide thickness there is a higher current density, and therefore $\mathrm{Al}^{3+}$ dissolves faster. This results in a further decrease of oxide thickness in these areas and thus enhances this effect and leads to the formation of pores.

The $\mathrm{Al}_{2} \mathrm{O}_{3}$ growth rate is dependent on the transport of $\mathrm{O}^{2-}$ and $\mathrm{OH}^{-}$to the aluminium surface. The growth process has to be faster than the dissolution of aluminium, so the aluminium can only grow on the area of later pore rims.

Alumina has a lower density than aluminium which results in mechanical stress and repulsive forces during the $\mathrm{Al}_{2} \mathrm{O}_{3}$ formation. The stress is reduced by the formation of hexagonal structures. With growing pore depth the pore bottoms are gradually directed into a hexagonal pattern. Now, after the initial $\mathrm{Al}_{2} \mathrm{O}_{3}$ layer is removed, an aluminium surface with hexagonally arranged pits is left behind. 
If the anodization is repeated on the pre-structured aluminium surface, pores are formed in the regions of the pits leading to a hexagonal pattern of highly ordered pores (Fig. 2.10).

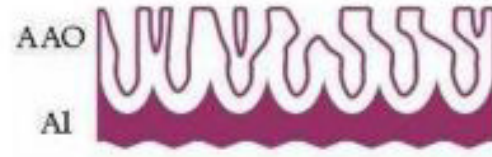

(a)

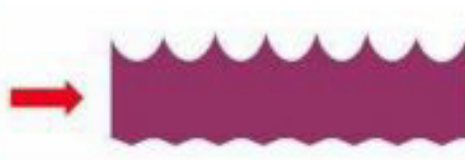

(b)

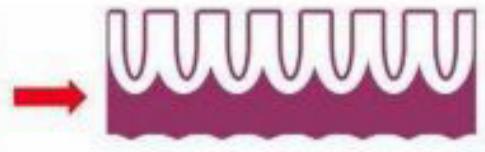

(c)

Fig. 2.10: Formation of highly ordered pores. (a). Schematic representation of the two-step anodization procedure to obtain highly-ordered aluminium oxide. Anodic ionization of aluminium in an electrolyte bath leads to growth of nanoporous aluminium oxide. Due to variations in local field strength pores are formed that are disordered at the top while the degree of order increases with time and depth of the pores. (b). Removal of the aluminium oxide layer leads the formation of a highly ordered aluminium surface. (c) A second anodization step of the pre-structured aluminium template leads to homogeneous local field strengths and therefore to homogeneous highly-ordered porous aluminium oxide.

It was shown that a pre-structured surface was prerequisite for the formation of highly ordered pores [93]. In this thesis, prestructure was achieved by anodization of the Al followed by a removal of the $\mathrm{Al}_{2} \mathrm{O}_{3}$ leading to a hexagonal pre-structured $\mathrm{Al}$ surface. Subsequent anodization then led to the formation of highly ordered pores arranged in a honeycomb structure. Finally, the remaining Al was dissolved in an acidic copper solution to obtain transparent aluminium oxide foils.

\section{Used Parameters: Two-step anodic oxidation}

\section{$\underline{\left.1^{\text {st }} \text { Anodization (step } 3\right)}$}

The polished Al foils were mounted to the chamber, which was filled with pre-cooled $0.3 \mathrm{M}$ oxalic acid at a temperature of $1.5^{\circ} \mathrm{C}$. The anodization was started by application of $40 \mathrm{~V}$ for $2.5 \mathrm{~h}$ at a temperature of $1.5^{\circ} \mathrm{C}$, leading to a preordered $\mathrm{Al}_{2} \mathrm{O}_{3}$ layer.

\section{$\underline{\text { Removal of the first } \mathrm{Al}_{2} \underline{\mathrm{O}}_{3} \text { layer (step } 4 \text { ) }}$}

The $\mathrm{Al}_{2} \mathrm{O}_{3}$ was removed by incubation in $5 \% \mathrm{H}_{3} \mathrm{PO}_{4}$ for $2-3 \mathrm{~h}$, resulting in a remaining prestructured Al surface.

$\underline{2^{\text {nd }} \text { anodization (step 5) }}$

The Al was anodized a second time under the same conditions as described above in which the pre-structure led to a higher ordered $\mathrm{Al}_{2} \mathrm{O}_{3}$ layer

\section{$\underline{\text { Selective Al removal (step 6) }}$}

The remaining $\mathrm{Al}$ on the backside of the $\mathrm{AAO}$ was selectively dissolved in an acidic copper solution $\left(17 \mathrm{~g} / \mathrm{L} \mathrm{CuCl} 2\right.$ in $\left.1: 1 \mathrm{H}_{2} \mathrm{O} / \mathrm{HCl}\right)$ while keeping the $\mathrm{Al}$ support on the edges of the 
AAO to prevent breaking of the chip. To obtain this, the front side of the hip was mounted to a glass slide with spacers between the chip and the glass. The Chip was sealed using an epoxide glue (Norland optical adhesive NOA 83H, 2 drops dissolved in $1 \mathrm{~mL}$ tetrahydrofuran, Norland Products, Cranbury, USA) while the regions to be dissolved were left free.

\subsubsection{Transfer to glass support and pore widening}

The obtained AAO was very thin and easily breakable. Therefore, the AAO was glued to a glass support to improve handling in the subsequent silanization process and during measurements.

To increase the average pore diameter from $25 \mathrm{~nm}$ to $70 \mathrm{~nm}$, the pores were widened by incubation in phosphoric acid. The final pore diameter $\mathrm{d}_{0}$ after the incubation time $\mathrm{t}_{0}$ can be calculated using the following equation [94]:

$$
d_{0} \approx\left(0.75 \cdot t_{1}+25\right) n m \quad \text { Eq. } 4
$$

\section{Used Parameters}

Adhesive fixation of the AAO to a glass support (step 7)

Square glass chips with a side length of $0.5 \mathrm{~cm}$ were used as a support for the alumina foils. Onto each of these chips a single piece of alumina was glued. The glass chips were cut from microscope slides with a glass cutter. Before cutting, the slides were cleaned in an alkaline glass cleaning solution (Hellmanex). The slides were rinsed with water and cleaned twice in an ultrasonic bath for $15 \mathrm{~min}$.

The microscope slides were cut into slides with an edge length of $0.5 \mathrm{~cm}$, then the glass slides were scratched on the back with a glass cutter to form squares. After cutting, the slices were cleaned with ultrapure water and ethanol, and stored at room temperature.

Directly before gluing, the slides were again cleaned with ethanol to be free of dust and grease. With this preparation several foils of porous alumina could simultaneously be glued to the glass support and after functionalization individual substrates of aluminium oxide on glass support could be easily broken from the slide as square chips.

The AAO foils were fixed on the glass with an optically transparent single component liquid adhesive (Norland Optical Adhesive $83 \mathrm{H}$ ) that cures to a polymer when exposed to ultraviolet light. Before application to the glass side, 2 drops of the adhesive were diluted in $1.5 \mathrm{~mL}$ tetrahydrofuran and applied to the glass slide using a Pasteur pipette. The foils were carefully moved onto the slide to remove entrapped air. Subsequently, the adhesive was cured with ultraviolet light ( $254 \mathrm{~nm}$ und $365 \mathrm{~nm}$ ) for at least $2 \mathrm{~h}$. 


\section{$\underline{\text { Pore widening (step } 8)}$}

Before incubation, the bonded substrates on the glass support were rinsed with p.a. ethanol to remove entrapped air in the cavities of the alumina. The substrates were incubated in $5 \% \mathrm{vol}$. $\mathrm{H}_{3} \mathrm{PO}_{4}$ for 50 min while stirring at $20^{\circ} \mathrm{C}$. Under these conditions the pore diameter increased by an average of $0.75 \mathrm{~nm} / \mathrm{min}$.

After incubation, the substrates were rinsed with water and p.a. ethanol to remove $\mathrm{H}_{3} \mathrm{PO}_{4}$, and dried under a nitrogen stream.

\subsubsection{Silanization}

Through orthogonal gas phase silanization it is possible to selectively generate a hydrophilic functionalization on the pore rims. Since the adhesive forces are very high on nonfunctionalized porous alumina, vesicle spreading does hardly lead to lipid bilayer formation [91],[95]. Therefore the surface was functionalized hydrophilically through silanization with (3-Mercaptopropyl)triethoxysilane (Fig. 2.11).

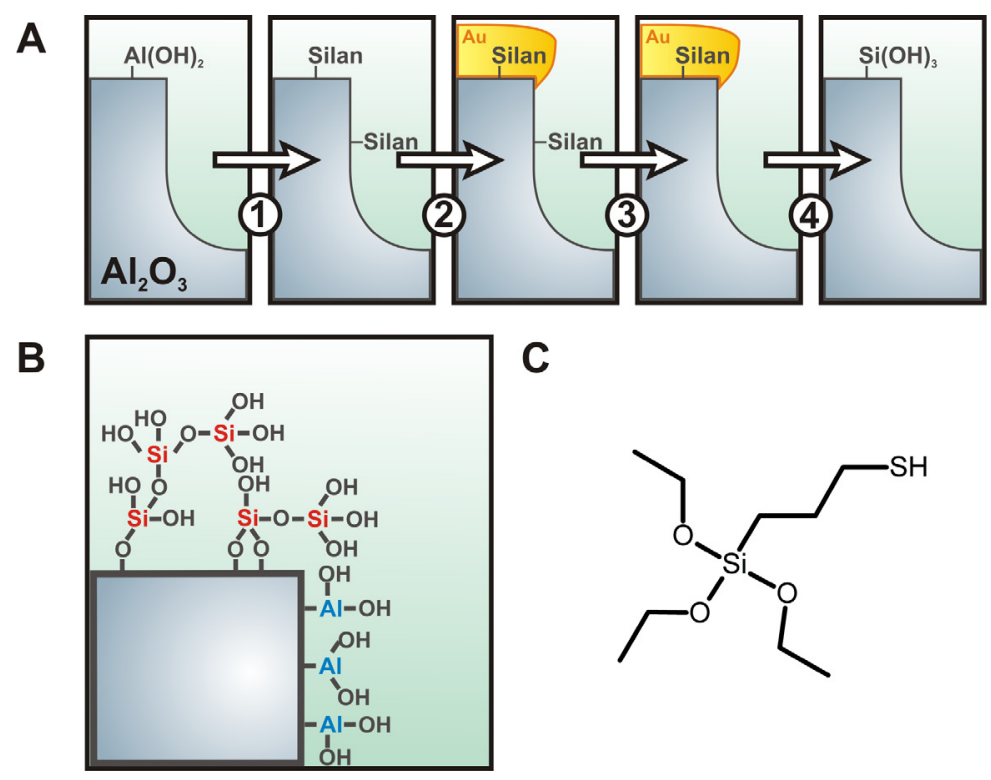

Fig. 2.11: Silanization of porous anodic aluminium oxide substrate. (A) Schematic representation of the orthogonal functionalization of alumina: (1) silanization with 3-mercaptopropyl triethoxysilane, (2) Evaporation of a thin gold layer (approx. $10 \mathrm{~nm}$ ), (3) selective removal of the functionalization on the pore walls by incubation in $\mathrm{O}_{2}$ and Ar plasma, (4) removal of the gold layer with $\mathrm{KI} / \mathrm{I}_{2}$ and hydrophilization by the application of $\mathrm{O}_{2}$ plasma. (B) The pore rims are functionalized by hydrophilic silanol groups while the pore interior is unfunctionalized. (C) Structure of 3-mercaptopropyl triethoxysilane. Image Source: [1],[2].

In a first step, the aluminium oxide surface is silanized by gas phase silanization. By gold evaporation the functionalization is protected on the pore rims. In this state the substrates can be stored.

By a combination of Argon and $\mathrm{O}_{2}$ plasma treatment, the functionalization in the pores can be selectively removed, while the functionalization on the surface is still protected. Gold can be 
removed by $\mathrm{KI} / \mathrm{I}_{2}$. Finally the silanes were oxidized by oxygen plasma treatment. This step was carried out directly before the preparation of the pore-spanning lipid bilayers and is described in detail in chapter 2.2.4.

\section{Used Parameters}

\section{Gas phase silanization (step 9)}

In the first step the bonded substrates were cleaned and the number of hydroxyl groups was increased by applying an oxygen plasma (Plasma Cleaner PDC 32 G-2, Harrick, Ithaka, NY, USA) for $1 \mathrm{~min}$. In the second step the substrates were mounted in a glass staining jar, and $50 \mu \mathrm{L}$ of mercaptopropyl-triethoxysilane (SHTES) was added in a glass test tube inside the chamber. The chamber was closed with the glass cover and sealed using vacuum tape (3M Electrical Tape 102087, Scotch ${ }^{\mathrm{TM}}$, St. Paul, MN, USA). For silanization the chamber was put into a vacuum drying oven. After $10 \mathrm{~min}$ incubation at $135^{\circ} \mathrm{C}$, vacuum was applied for at least $3 \mathrm{~h}$ at $135^{\circ} \mathrm{C}$.

Directly after silanization the substrates were cooled to room temperature and about $10 \mathrm{~nm}$ of gold was deposited onto their surface under high vacuum (Coating System MED020, Bal-Tec (Leica, Wetzlar) to protect the silanization (2.2.3.4) (Due to the pore diameter only pore rims were covered with a colloidal gold layer, while the pore interior was left uncovered). The covered substrates could be stored for several weeks at room temperature.

\subsubsection{AAO surface priming}

The selective removal of the silane (Fig. $2.11 \mathrm{c}$ ) in the pore interior was carried out directly before the preparation of the pore-spanning lipid bilayers. One glass chip was broken off the gold protected, silanized AAO slide.

$\underline{\text { Selective removal of silanization in the substrate pore interior }}$

In this step an $\mathrm{Ar}$ and $\mathrm{O}_{2}$ plasma was applied to the gold protected AAO substrate. This allowed selective removal of the silanization in the pore walls while the functionalization was preserved on the pore-rims under the protective gold layer.

The functionalization in the pore interior was removed by application of 1 min Ar plasma, followed by 1 min of $\mathrm{O}_{2}$ plasma (Plasma Cleaner PDC 32 G-2, Harrick, Ithaca, NY, USA).

\section{$\underline{\text { Removal of the protective gold layer }}$}

For the removal of the protective gold layer, the AAO substrate was immersed in $\mathrm{I}_{2} / \mathrm{KI}$ solution $\left(0.5 \%(\mathrm{w} / \mathrm{v}) \mathrm{KI}, 0.125 \%(\mathrm{w} / \mathrm{v}) \mathrm{I}_{2}\right)$ for a maximum of $10 \mathrm{~s}$. Subsequently, the substrate was immediately dipped in a beaker with $\mathrm{H}_{2} \mathrm{O}$ and rinsed with $\mathrm{H}_{2} \mathrm{O}$ to remove residual $\mathrm{I}_{2} / \mathrm{KI}$. After this the chip was incubated in $p$.a. ethanol for $1 \mathrm{~min}$ to remove water 
from the cavities, and after rinsing with p.a. ethanol the substrate was dried in a nitrogen stream.

\section{$\underline{\text { Silane oxidation to silanol }}$}

To assure a sufficient hydrophilic surface, the silanes were oxidized to silanoles by application of $\mathrm{O}_{2}$ plasma for 1 min (Plasma Cleaner PDC 32 G-2, Harrick, Ithaka, NY, USA).

\subsubsection{AAO substrate mounting}

Fixation of the substrate to the measuring chamber

For the measurements a Petri dish was used as a measuring chamber. To prevent air inclusions in the cavities, the AAO substrate was incubated in p.a. ethanol for $1 \mathrm{~min}$, after which the back of the chip was dried under a nitrogen stream in order to ensure good adhesion. Then the AAO substrate was fixated to the Petri dish bottom with an adhesive strip. To assure good adherence pressure was by carefully exerted on the edges of the substrate (Fig. 2.12 step 1). Direct contact of ethanol with the strip had to be prevented since this reduced the adhesion.

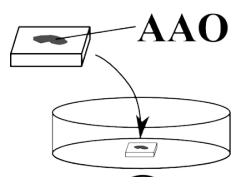

(1)

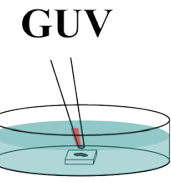

(4)

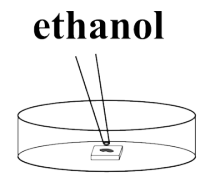

(2)

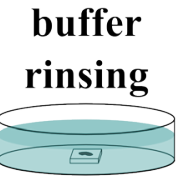

(5)

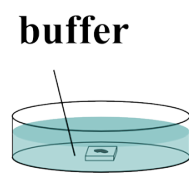

$(3)$

fluorophore

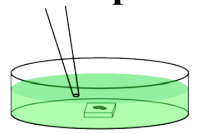

(6)

Fig. 2.12: Schematic representation of the AAO substrate preparation in the reaction chamber. (1) The AAO substrate on the glass chip is glued to a Petri dish with an adhesive strip. (2) Ethanol is added to the substrate and (3) exchanged with buffer to prevent air entrapment. (4) The GUV solution is pipetted to the substrate and incubated for spreading. (5) After spreading residual GUVs and lipid material is removed by buffer exchange. (6) Finally, the volume is adjusted and a water-soluble fluorescent dye can be added to the measuring chamber.

\section{$\underline{\text { Buffer addition }}$}

The net weight of the measuring chamber with the substrate was determined to allow for the calculation of the amount of buffer added later in the procedure via weight measurements. A drop of p.a. ethanol was added to the substrate and incubated for $1 \mathrm{~min}$. The procedure was repeated once. Again, ethanol contact with the adhesive had to be prevented and the volume needed to be adjusted accordingly.

The chamber was rinsed by carefully pouring $50 \mathrm{~mL}$ buffer over the substrate while preventing AAO contact with air bubbles. After rinsing, excess buffer in the chamber was 
removed to leave a residual volume of approximately $3 \mathrm{~mL}$ in the chamber (Fig. 2.12 steps 2 and 3).

\subsubsection{Preparation of silicon-based porous substrates}

\subsubsection{Open silicon nitride pores}

Open pore silicon nitride substrates were prepared through photolithographic methods by Fluxxion (Eindhoven, Netherlands), from where they were obtained commercially. The substrate featured a pore diameter of $1.2 \mu \mathrm{m}$ with a $800 \mathrm{~nm}$ layer of silicon nitride on a base of silicon. Substrate surfaces were functionalized hydrophilically to allow adhesion of lipids as described in 2.2.3.3 and 2.2.3.4.

\subsubsection{Closed silica pores}

Closed pore silicon substrates were obtained from Dr. Siegfried Steltenkamp (Micro System Technologies, Center of Advanced European Studies and Research, CAESAR, Bonn). The substrate featured a pore diameter of 3.5-5.5 $\mu \mathrm{m}$ and a depth of $6 \mu \mathrm{m}$ and was coated with silicon dioxide after structuring. Substrate surfaces were functionalized hydrophilically to allow adhesion of lipids as described in 2.2.3.3 and 2.2.3.4.

\subsubsection{Hydrophilic functionalization with hydroxyl groups}

To facilitate adhesion of the amphiphatic lipids on the porous substrates, hydrophobic as well as hydrophilic functionalization are suitable methods. Silicon-based substrates were primed hydrophilically, which allowed the head groups of the lipids to adhere to the substrate surface through dipole-dipole interactions.

Initially, the substrate surface was primed with low pressure oxygen plasma. The ions of the plasma react with any surface contaminants such as hydrocarbons, oils and fats, and reaction products are carried away from the substrate through the gas stream. The oxygen plasma also leads to the removal of atoms from the substrate surface. Here, water from the surrounding atmosphere can adhere through chemisorption, resulting in a highly hydrophilic surface [96].

\subsubsection{Hydrophilic functionalization with thiols after gold coating}

Alternatively, the porous substrate was coated with a thin film of gold. This allowed for the subsequent chemisorption of hydrophilic thiols.

First, the substrate was cleaned under Argon plasma for 1 min and sputter-coated with $2.5 \mu \mathrm{m}$ titan (0.05 mbar, $40 \mathrm{~mA}$, Cressington Sputter Coater 108auto) to achieve better adhesion of the subsequent gold coating. Then, the substrate was gold-coated through thermal evaporation. The apparatus used in this work (MED 020, Bal-Tec AG, Liechtenstein) was operated with a tungsten scoop that was heated through current passage of $4 \mathrm{~V}$ and 60-70 A. 
The substrate was placed perpendicularly to the vaporization source at $10 \mathrm{~cm}$ distance and covered with a $30 \mathrm{~nm}$ gold layer at $0.5-1.5 \mathrm{~nm} / \mathrm{s}(55-65 \mathrm{~A})$.

Gold atoms that are ejected from the target surface through thermal energy (as is the case in thermal evaporation technique) show radial trajectories from their source. Due to their high average length of path, they can be expected to reach the substrate directly without being deflected during their flight. Because of this, they reach the substrate in a perpendicular angle in the used setup, so that adhesion of gold particles on the pore interior is less likely. This is why this coating mthod was favored over cathode ray atomization (sputtering) since sputtering may lead to undesired displacement of gold in the interior of the substrate pores. This is due to the short length of path of the gold atoms emitted by the sputterer, and resulting trajectory changes as gold atoms collide with the atoms of the carrier gas during their flight.

Immediately after gold coating, the substrate was incubated with in $3 \mathrm{~mL} 1 \mathrm{mM} 1$ Mercaptohexan-6-ol, dissolved in n-propanol, over night at $4{ }^{\circ} \mathrm{C}$.

\subsubsection{Spreading of giant unilamellar vesicles}

Sucrose-filled giant unilamellar vesicles (preparation described in chapter 2.2.1) were added immediately to the substrate after the procedures described in 2.2.2.7, 2.2.3.3, or 2.2.3.4.

\section{Addition of the giant unilamellar vesicle solution}

GUVs were added to the substrate from a stock solution. The vesicles sink towards the bottom of the dish due to the higher density of their interior and rupture after they make contact with the AAO surface.

For the formation of pore spanning membranes, $60 \mu \mathrm{L}$ GUV solution were added to the substrate and the substrate was incubated in a dark chamber for $10 \mathrm{~min}$ at room temperature (Fig. 2.12 step 4).

\section{Removal of excess vesicles by buffer exchange}

After GUV spreading, excess vesicles and lipid material in the solution were removed by buffer exchange. This was done by carefully removing $1 \mathrm{~mL}$ of buffer in the volume over the substrate, followed by the addition of $1 \mathrm{ml}$ fresh PBS-buffer (Fig. 2.12 step 5). The procedure was repeated 10x. Again, substrate contact with air bubbles and excessive buffer flow directly on the membrane surface were avoided, since this can destroy the freshly formed pore spanning membranes.

\section{Addition of fluorescent dye}

To determine the buffer volume (assuming a buffer density of $1 \mathrm{~g} / \mathrm{ml}$ ), the chamber was reweighted and the net weight was deducted. Accordingly, buffer was added or removed to a final buffer volume of $3 \mathrm{~mL}$ to match the microscope setup. Fluorescent dye was then added from a stock solution to the desired end concentration (pyranine: $1 \mathrm{mM}$; Dex40: $8.3 \mu \mathrm{M}$; 
Dex70: $4.8 \mu \mathrm{M}$; Fig. 2.12 step 6). The fluorescently labeled pore-spanning membranes could now be observed under a fluorescent microscope.

\subsection{Analytical Methods}

\subsubsection{Fluorescence Microscopy}

Fluorescence microscopy allows for the investigation of fluorescent biomolecules and cell components in the nanometer range under physiological conditions.

With the help of fluorescent labeling by covalent binding of fluorophores to target molecules or recombinant expression of fluorescent fusion proteins, a wide range of biomolecules and cell compartments can be investigated.

The fluorophores in the sample are excited by light matching the excitation wavelength, and emit light of a longer wavelength. The emitted light is separated from the excitation light and directed to an ocular or a detector. The optics of the microscope produce a magnified image of the fluorescent sample, which can then be detected.

\subsubsection{Fluorescence}

The spontaneous instantaneous light emission of matter after excitation with electromagnetic radiation is called fluorescence. A fluorescent molecule is referred to as a fluorophore.

Fig. 2.13 shows a typical fluorescence spectrum of the fluorophore Texas Red. The excitation band (blue) shows the wavelength range of light that can be used to excite the fluorophore, while the emission band (green) shows the wavelengths of the emitted light. Both bands show a peak at a distinct wavelength. The shift between the excitation and the emission peak is called Stokes Shift. 


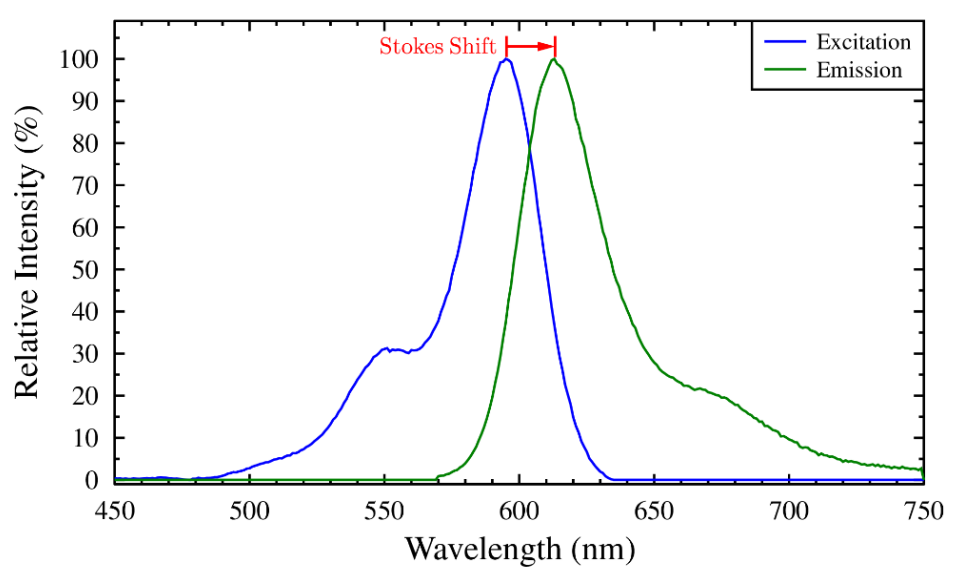

Fig. 2.13: Fluorescence spectrum of Texas Red. Emission wavelengths (green) are shifted towards longer wavelengths in comparison with the excitation wavelengths (blue). The difference between the excitation and emission peak is the Stokes shift.

With the help of fluorescence spectra (Fig. 2.13), the optimal selection of the fluorophores and optimization of the excitation and detection parameters in the experimental setup can be made.

\subsubsection{Fluorescence Spectra: Franck-Condon-Principle}

The shape of a fluorescent spectrum is determined by the energy states of the fluorophore. The energetic state of lowest possible energy is referred to as the ground state while all higher energetic states are called excited state.

The energy of a molecule is stored in forms of electronic, vibrational, rotational and translational energy. Electronic, vibrational and rotational energies are quantized, meaning they can only take certain discrete energy levels. The energetic state of a molecule can be changed by electronic excitation (change in electron density by energy absorption), vibrational excitation (vibration of nuclei) and rotational excitation (rotation of the molecule). 


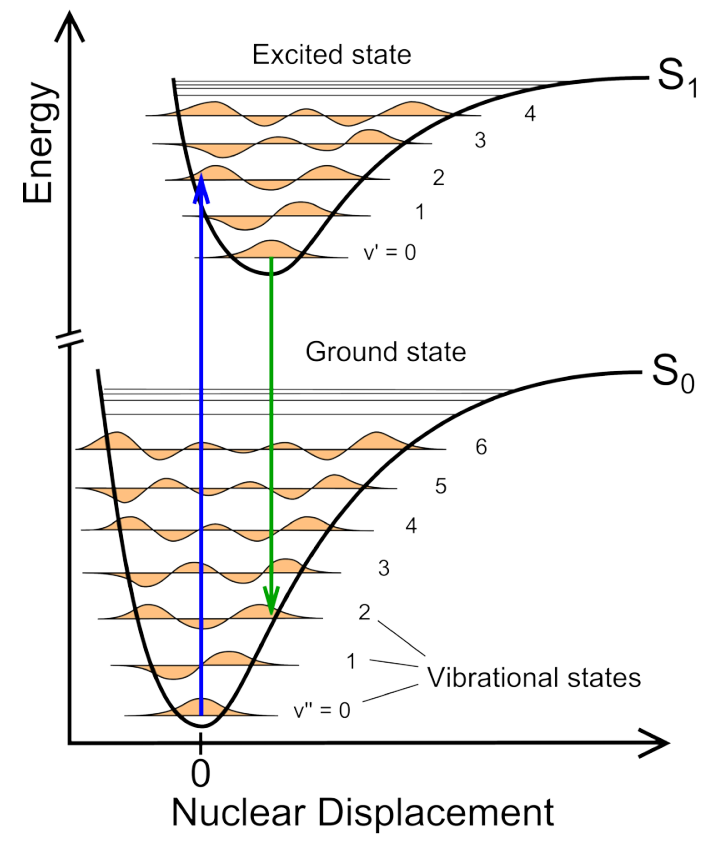

(a)

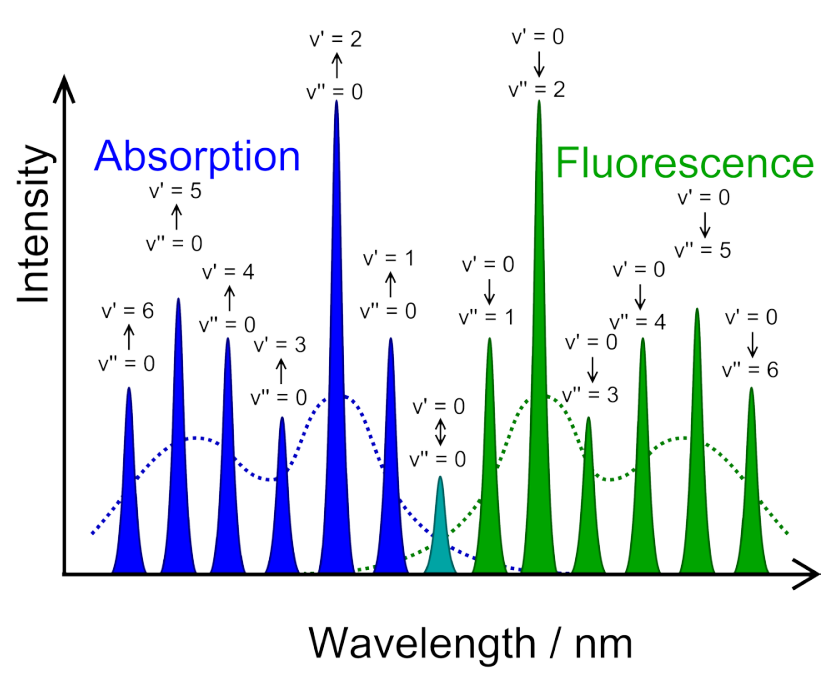

(b)

Fig. 2.14: Schematic representation of the Franck-Condon-Principle (a) and the resulting fluorescence spektrum (b) Image Source (edited from)

The Franck-Condon-Principle is an approximation stating that an electronic transition most likely occurs without change of the nuclei positions. When a molecule is moved into another excited state, the transition is most likely when there is a maximum compatibility of the vibrational wave functions (similar to the wave function at the original nuclear position).

Fig. 2.14 exemplifies that, for a vibronic transition between these states, energy must be absorbed (blue arrow) or released (green arrow). This is only possible by absorption or release of quantized energy packages matching the characteristic distances between the molecular energy states.

This presentation is directly linked to the absorption spectra. An absorption spectrum shows the probability with which a photon is absorbed by the fluorophore as a function of its wavelength. The corresponding emission spectrum shows the probability with which wavelength a photon is emitted.

\subsubsection{Widefield Fluorescence Microscopy}

A fluorescence microscope is variation of a classical light microscope that uses fluorescence to create a magnified image of a sample. Fluorescence microscopy uses visible light to excite the sample.

The principle setup of a widefield fluorescence microscope is shown in Fig. 2.15. 


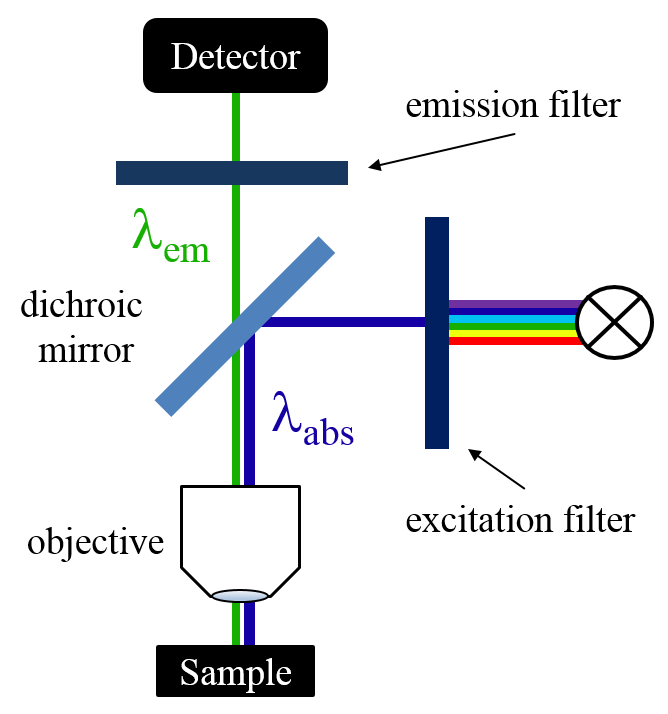

Fig. 2.15: Schematic drawing of the optics of a widefield fluorescence microscope.

The light source emits monochromatic light matching the excitation wavelength $\lambda_{\text {abs }}$ of the fluorophore. Monochromatic light can either be generated by monochromatic light sources (lasers or light emitting diodes, LEDs) or by spectral filtering of continuous light using excitation filters. A dichroic mirror reflects the light to the fluorescent sample which then emits light of the emission wavelength $\lambda_{\text {em. }}$. Most of the light with the wavelength $\lambda_{\text {abs }}$ is directly reflected back to the light source, while light with the wavelength $\lambda_{\text {em }}$ passes the dichroic mirror and can then be detected. A second filter ensures that only light with the wavelength $\lambda_{\mathrm{em}}$ is detected.

Optical resolution and limitations of the widefield fluorescence microscope

The maximum resolution of a fluorescence microscope is determined by Abbe's law

$$
d=\frac{\lambda}{2 n \sin \alpha}=\frac{\lambda}{2 N A}, \quad \text { Eq.5 }
$$

The diffraction limit of a microscope is dependent on the wavelength $\lambda$, the medium's refractive index $n$ and $\alpha$ one half of the angular aperture. The latter are usually combined to the numerical aperture $N A$. The diffraction limit for a fluorescence microscope results is $1 / 2 \lambda_{\text {ex }}$ (excitation light wavelength) that is approximately $200 \mathrm{~nm}$ for visible light.

For widefield microscopy, simultaneous detection of light from out of focus planes leads to a limitation in spatial resolution. Additionally, the detected images are an overlay of light from the sharp focal plane and blurred out of focus light which leads to a decrease in image quality. Confocal microscopy is a technique to overcome the limitations by out of focus light and increase spatial resolution. 


\subsubsection{Confocal Laser Scanning Microscopy (CLSM)}

Confocal laser scanning microscopy uses pinholes to improve the spatial resolution of the fluorescence microscopy. The resolution is improved by minimizing the detection of out of focus light.

This is done by a combination of selective excitation of a narrow focal plane and selective detection of light from this focal plane. Laser light and a pinhole allow the generation of a point-like light that can selectively excite a narrow focal place of interest. Simultaneously, out of focus light is blocked by a second pinhole. A typical beam path is shown in Fig. 2.16.

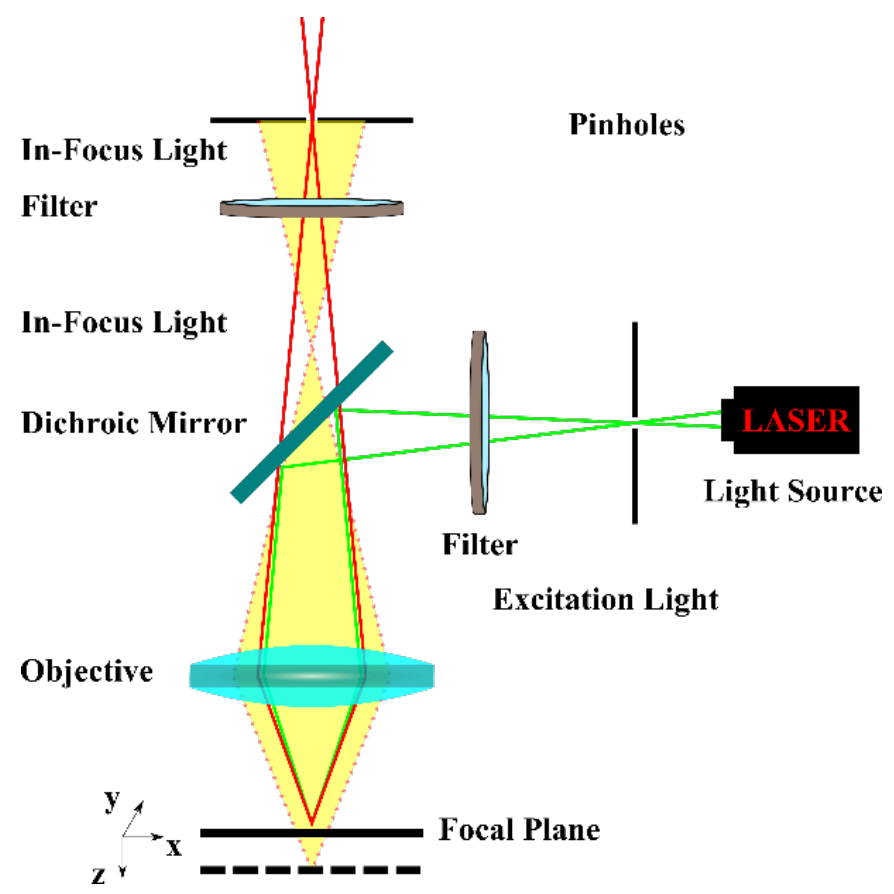

Fig. 2.16: Schematic representation of a confocal laser scanning microscope. A laser in combination with a pinhole generates monochromatic point-like light with the wavelength $\left(\lambda_{\mathrm{abs}}\right)$. A dichroic mirror reflects the light onto the sample, and light of longer wavelength is emitted. The sample is scanned point-by-point through the use of scan mirrors. Emission light passes the dichroic mirror, while most excitation light is reflected back to the light source. Out of focus light is removed by a pinhole.

The used parameters for the confocal laser scanning microscope are described in chapter 2.4.3.

\subsection{Antimicrobial peptide permeabilization assay}

The principle of the used permeabilization assay is the observation of the ability of a watersoluble fluorescent dye to pass through a lipid bilayer, e.g. the interior of a vesicle or a porespanning membrane (Fig. 2.17). 


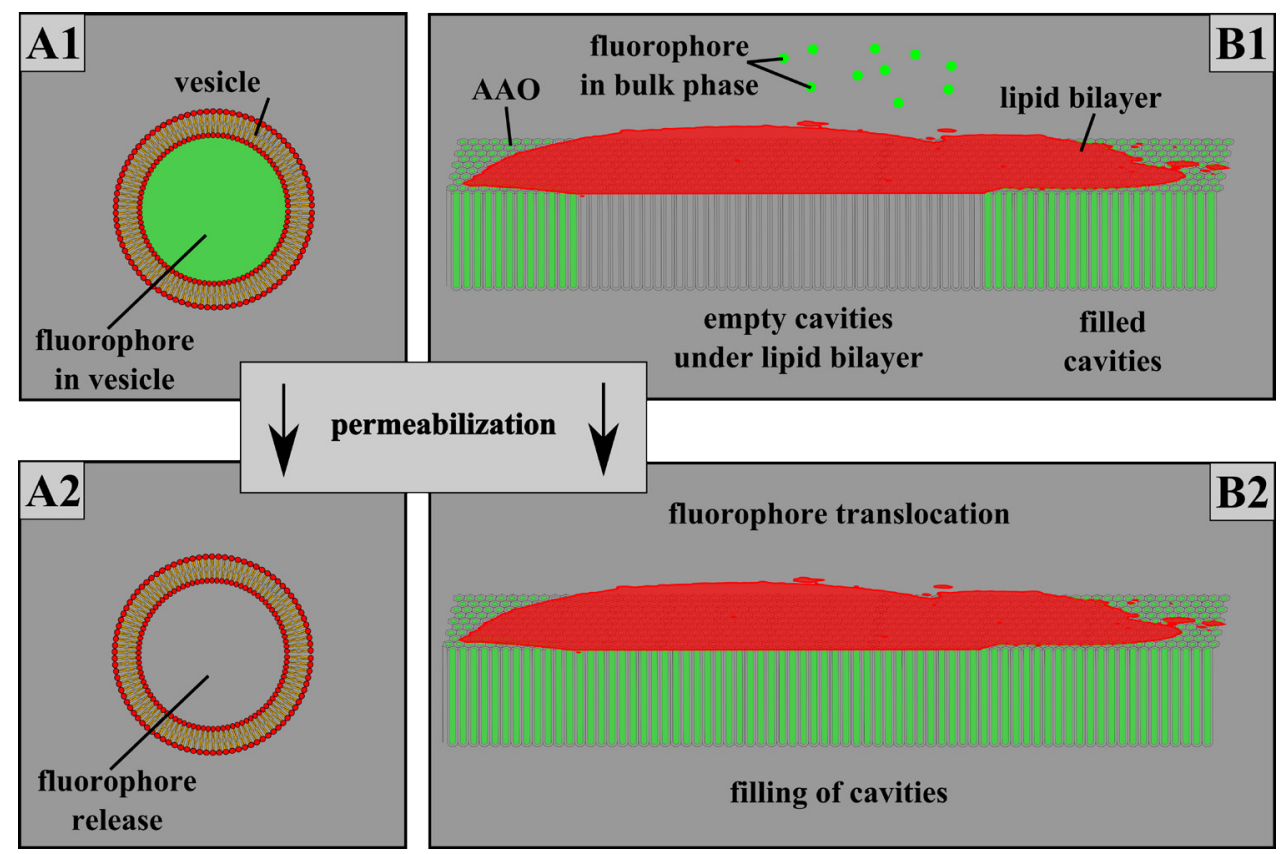

Fig. 2.17: Comparison of vesicles and pore-spanning membrane permeabilization assays. In vesicles, a fluorescent dye may be included (A1). After addition of a permeating agent the fluorophore is released and the change of fluorescence can be detected (A2). For pore-spanning lipid bilayers, the fluorescent dye is excluded from the bulk phase by a lipid bilayer patch (B1). After addition of a permeating agent the fluorophore is able to translocate into the cavities under the lipid bilayer (B2), which can be detected by fluorescence microscopy.

Investigation of the mode of lipid bilayer lysis and pore formation can be experimentally approached by appropriate selection of fluorescent dyes. Bulky fluorescent dyes like labeled dextrans may behave differently from relatively small dyes like fluorescein or pyranine upon formation of membrane leaks, depending on the size of formed pores or the occurrence of membrane lysis. In case of pore formation, the pore diameter and time dependence of pore formation can be estimated by using dyes with known stokes radii.

A typical measurement for the investigation of permeabilization of a lipid bilayer membrane is the determination of the AMP threshold concentration. This is the AMP concentration below which no translocation of soluble fluorescent dye across the membrane is observable. Also, a change in threshold concentration depending on dye size can be monitored.

By using the assays described in the next chapters, the dependency of permeabilization events on lipid composition, AMP concentration, fluorescent dye radius, time, and buffer composition can be investigated.

\subsubsection{Membrane preparation and localization for fluorescence microscopy}

POPC lipid bilayer membranes with $0.5 \%$ Texas Red DHPE were prepared as described in chapter 2.2. A second water-soluble fluorescent dye was added to the buffer surrounding the 
membrane as indicated in the respective experiments. The chamber was placed under a water immersion objective of the fluorescence microscope, and imaged continuously.

Fluorophore inflow into the substrate pores upon addition of AMPs was checked in dependency on AMP concentration and time. Each measurement for a distinct concentration shown in the results represents an independent experiment, and implies membrane preparation on different substrate entities since the membrane cannot be regenerated after AMP addition.

For each experiment the membranes were prepared under the same conditions. After membrane preparation, membrane patches were located using the epifluorescence setup of the confocal laser scanning microscope.

After successful localization of a lipid bilayer patch, the fluorescence of the water-soluble dye was monitored in a focal plane underneath the lipid bilayer patch using confocal laser scanning microscopy.

\subsubsection{Fluorescence imaging}

For selective detection of the fluorescence under the lipid bilayer patches, three-dimensional z-stack fluorescence images were taken using confocal laser scanning microscopy. A schematic overview for the image acquisition is presented in the Results section Fig. 3.7.

For z-stack imaging, 2D-images with constant position in $x-y$ coordinates were automatically scanned subsequently while varying the focus in $z$-direction in a defined volume of the lipid bilayer patches. From the images, a 3D z-stack image was calculated using the ZEN software.

The time resolution of z-stack imaging is limited by the scanning speed of the CLSM. To acquire data with adequate time resolution, a single line in $x-y$ position crossing the membrane patch was recorded representatively for the whole patch, and stacked ("z-line scan"). The resulting image equals a cross-section of a 3D z-stack image.

\subsubsection{Microscope setup and detection parameters}

An upright confocal laser scanning microscope was used for the experiments. An LSM 710 laser scanning unit was combined with an Examiner.Z1 stand (Carl Zeiss, Jena, Germany).

Water immersion objectives with 20x (W Plan-Apochromat 20x/1.0) and 63x (W PlanApochromat 63x/1.0) magnification were used (Carl Zeiss, Jena, Germany).

Zeiss Efficient Navigation (ZEN) software 2012 SP1 (black edition) was used for data acquisition and image processing (Carl Zeiss MicroImaging $\mathrm{GmbH}$ ). 


\section{$\underline{\text { Excitation and Detection }}$}

TexasRed DHPE was excited at $\lambda_{\mathrm{ex}}=594 \mathrm{~nm}$ and the emission was detected at $\lambda_{\mathrm{em}}=605-690$ $\mathrm{nm}$. Pyranine and FITC-labeled dextrans were excited at $\lambda_{\mathrm{ex}}=488 \mathrm{~nm}$ and the emissions were detected at $\lambda_{\mathrm{em}}=490-580 \mathrm{~nm}$.

Two-dimensional imaging

Two-dimensional images were taken as stated with a water immersion objective W PlanApochromat 63×/1.0 NA (Carl Zeiss, Jena, Germany).

Overview images displaying large areas of the AAO chip were taken with a W PlanApochromat 20×/1.0 NA water immersion objective (Carl Zeiss, Jena, Germany) as stated.

Three-dimensional imaging (z-stack and z-line imaging)

Z-stack images were taken with a water immersion objective W Plan-Apochromat $63 \times / 1.0$ NA (Carl Zeiss, Jena, Germany).

\section{$\underline{\text { Z-line time series }}$}

Z-line images were taken continuously throughout a time interval of $30 \mathrm{~min}$. After $>10 \mathrm{~min}$ equilibration, the antimicrobial peptide (as indicated) was pipetted into the chamber under continuous stirring to a final peptide concentration as indicated in the respective experiments.

\subsubsection{Data acquisition and analysis with MATLAB}

A typical course of dye inflow after peptide addition is characterized by a short lag phase after which the filling starts, followed by a gradual filling of the cavities that converges to a maximum, depending on the used peptide and peptide concentration.

The time course of dye inflow can be observed as change in fluorescence intensity $\left[\mathrm{I}_{\mathrm{ROI}}(\mathrm{t})\right]$ in a region of interest (ROI) under the membrane patch. Additionally, the intensity of a ROI in non-sealed cavities $\left[\mathrm{I}_{\text {ref }}(\mathrm{t})\right]$ is set as a reference for complete pore filling (Fig. 3.12).

The relative fluorescence intensity $\left[\mathrm{I}_{\mathrm{rel}}(\mathrm{t})\right]$ as a function of time $\mathrm{t}$ is calculated by normalizing $\mathrm{I}_{\mathrm{ROI}}(\mathrm{t})$ to the fluorescence intensity at $\mathrm{t}=0\left(\mathrm{I}_{0}\right)$, and standardizing against $\mathrm{I}_{\text {ref }}(\mathrm{t})$ using the following equation:

$$
I_{\text {rel }}(t)=\frac{I_{\mathrm{ROI}}(t)-I_{0}}{I_{\text {ref }}(t)-I_{0}}
$$

A visual explanation is given in the Results section, Fig. 3.12.

To quantify the time course of the resulting sigmoidal curves, a Boltzmann function is fitted to the as shown in Eq.7: 


$$
I_{\text {rel }}(t)=\frac{I_{0}-I_{\text {max }}}{1+\exp \left\{\frac{t-t_{0}}{\tau}\right\}}+I_{\text {max }} \quad \text { Eq.7 }
$$

$\mathrm{I}_{\max }$ represents the maximum fluorescence intensity, reflecting maximum pore filling. The center time $t_{1 / 2}$ represents the time when $50 \%$ of the maximum fluorescence intensity is reached, and can be used as a measure for the lag time. Lag time describes the initial phase after AMP addition, where the fluorescence intensity does not change considerably. The tangent slope $(\tau)$ at $t_{1 / 2}$ describes the filling rate of the pores, a value that gives information about how fast the process of filling occurs.

These values can be determined in dependence on peptide concentration to estimate the speed of filling and the degree of filled cavities after the experiment.

The Matlab Script for fluorescence detection and drift compensation is disclosed in the appendix. 
Materials and Methods 


\section{Results}

Lipid membranes are discussed as the main target of antimicrobial peptide activity. In this work, pore-spanning lipid bilayers were evaluated for their suitability to investigate antimicrobial peptide activity.

Pore-spanning membranes are lipid bilayer patches on a porous solid support. These membranes feature the advantages of solid supported membranes in combination with additional aqueous compartments under the lipid bilayer with underlying pores.

Three different kinds of porous supports were tested: Open pores on a silicon nitride substrate, closed pores on a silica substrate, and nanoporous anodic aluminium oxide (AAO). The silica and silicon nitride pores were functionalized by gold evaporation followed by hydrophilic functionalization with thiols.

Potential membrane permeabilization events were investigated by detection of fluorescence dye translocation over the membrane in dependency on antimicrobial peptide concentration. Simultaneously, changes in morphology of the fluorescently labeled lipid bilayer were studied.

\subsection{Pore-spanning membrane systems on open silicon nitride pores}

Pore-spanning membranes on open silicon nitride pores were used as a tool to investigate the interaction of antimicrobial peptides with the lipid bilayer. This work explored whether silicon nitride substrates with pore-spanning membranes would be suitable materials for the development of a permeabilization assay to test antimicrobial peptides.

Silicon nitride chips with pores of $1.2 \mu \mathrm{m}$ diameter were used as support for the porespanning lipid bilayer. Substrates were evaporated with a thin gold-film, and a hydrophilic self-assembled monolayer on the surface was formed by incubation with mercaptohexanol to generate a hydrophilic surface.

Pore-spanning lipid bilayers were prepared by spreading POPC GUVs labeled with $0.5 \%$ Texas Red DHPE on open silicon nitride pores. Melittin was titrated into the supernatant buffer at rising concentrations of $0.1 \mu \mathrm{M}, 0.4 \mu \mathrm{M}, 1.1 \mu \mathrm{M}$ and $1.3 \mu \mathrm{M}$, and confocal microscopic images of Texas Red DHPE fluorescence were acquired at respective melittin concentrations. 


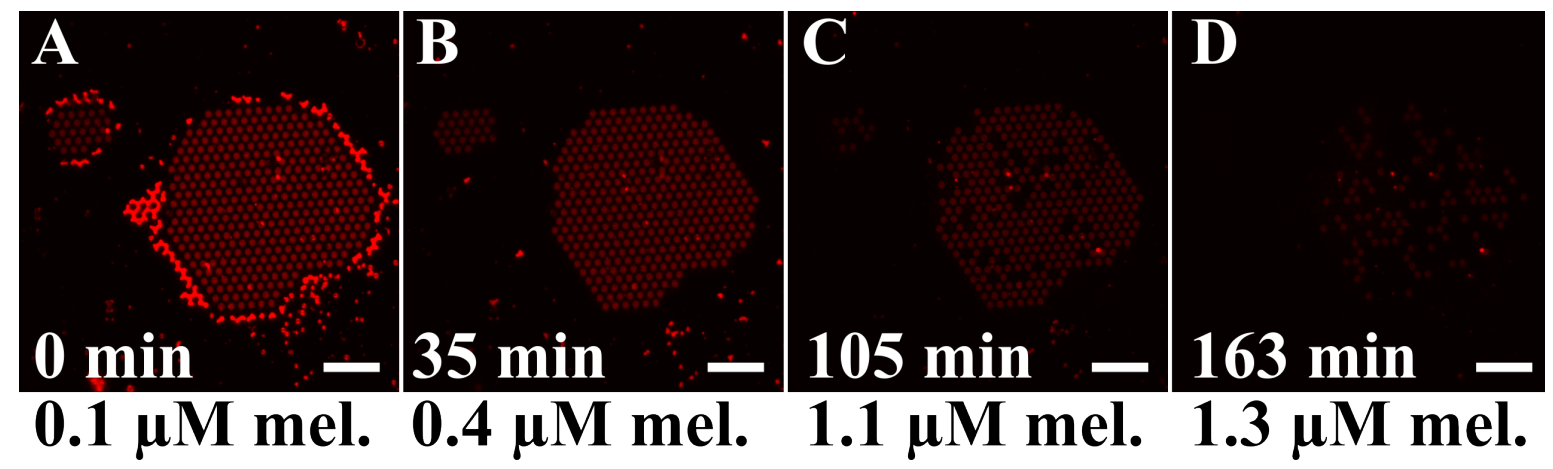

Fig. 3.1: Confocal laser scanning micrographs of Texas Red DHPE (red) labeled lipid bilayer patches on open silicon nitride pores with $1.2 \mu \mathrm{m}$ diameter. The antimicrobial peptide melittin was titrated into the supernatant buffer. A-D show representative states of the pore-spanning membrane at given time points and melittin concentrations. Addition of melittin led to the loss of fluorescence on the outer rim of the membrane patches, followed by decrease in fluorescence and rupturing of single pores up to complete loss of fluorescence. Scale bar: $20 \mu \mathrm{m}$.

Spreading of GUVs on silicon nitride support led to the formation of pore-spanning membranes. As shown in Fig. 3.1 A, GUVs spreading yielded heart-shaped membrane patches that were surrounded by excess lipid material. The bright intensity of the surrounding excess material indicates the formation of multilayers or aggregated lipid material. The gold quenching on the pore rims led to a visible hexagonal pattern where the red areas represent pore-spanning lipid bilayers over the pores, and dark red areas mark lipid membrane on substrate support. After addition of $0.4 \mu \mathrm{M}$ melittin (Fig. $3.1 \mathrm{~B}$ ), the aggregates of lipid material at the rim of the membrane patches began to disappear. In further progress, the fluorescence intensity of individual pores of the membrane patches disappeared altogether. This process can be seen for few pores in Fig. 3.1 C, and is more pronounced in Fig. 3.1 D. These observations suggest that the membranes over the respective pores had ruptured. 
A

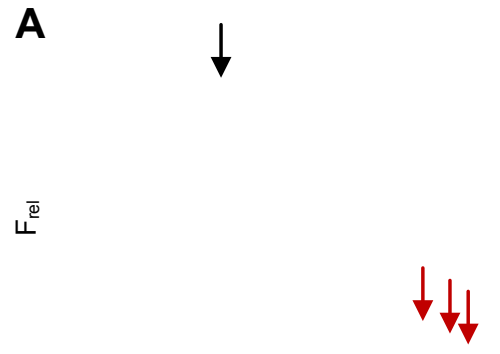

B

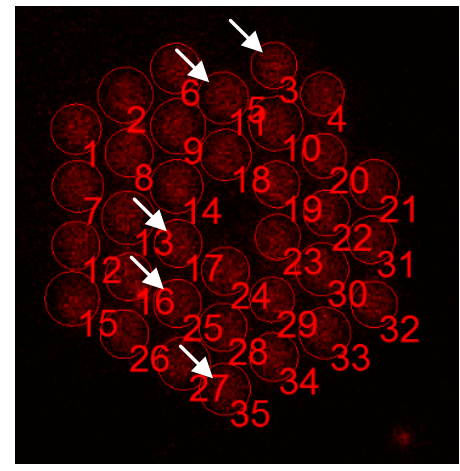

Fig. 3.2: Analysis of lipid bilayer fluorescence loss upon melittin addition. (A) Graphic representation of measured fluorescence values over time. Curves for several membrane areas over individual pores (B, arrows) are depicted. The addition of melittin after $32 \mathrm{~min}$ (A, black arrow) led to a simultaneous fluorescence decrease by two thirds of the original value. The rupture of the lipid bilayer on individual pores at varying time points is seen by the second fluorescence drop after 90-105 min (B, red arrows). It occured at different time points for each individual pore and led to a fluorescence decrease to background level.

Fig. 3.2 displays how the decrease of fluorescence intensity was monitored. Analysis of the fates of individual membrane patches allowed for the quantification of intensity decrease upon melittin addition, and rupture events as marked by a sudden fluorescence drop to baseline level. For this, ROIs were drawn encompassing individual free membrane sections over the silicon nitride pores (numbered circles, Fig. 3.2 B), and tracked over time. Each ROI represents the free membrane over a pore. The fluorescence signals of the membrane after addition of melittin were noisy and and did not yield data that allowed for interpretation of a possible permeability of the membranes prior to rupture. Furthermore, it was not possible to include a dye release assay upon membrane rupture. This would require the trapping of a fluorescent dye within the pores, which is not possible for open pores because any substance would escape through the bottom of the pores. To conclude, the readout for open silica pores was limited.

\subsection{Pore-spanning membrane systems on closed silica pores}

As could be seen from the first experiments of pore-spanning membranes on open silicon nitride pores, the assay only gave a yes/no readout for membrane rupture. It did not allow for evaluation of a potential membrane permeabilization before the rupture.

Due to this, the use of closed pores (pores with a sealed base) was considered. This would allow for filling of the pores with a dye which cannot permeate lipid membranes and could be trapped by the membrane on the pore surface. Upon membrane permeabilization or rupture, the trapped dye would be released with characteristic kinetics, allowing for an evaluation of the membrane condition. The translocation of the dye can be monitored by recording fluorescence changes over time within the lumen of the pore. Silica pores with a diameter of $3.5 \mu \mathrm{m}$ were chosen for this approach. The small hydrophilic pyranine was chosen as dye. 
The system was tested for three main features: Successful spreading of GUVs on the substrate and trapping of the aqueous dye below the formed planar membranes had to be confirmed. Then, the release of the dye from the pores at melittin concentrations that perforate, but do not lyse the membrane, was assayed. Lastly, membrane lysis at high melittin concentrations was monitored.

\subsubsection{GUV spreading}

In previous work it could be shown that the preparation of pore-spanning membranes on closed pores is possible [86]. However, these membranes formed only small patches, had a low degree of surface coverage on the substrate, and were speculated to form spherical protrusions on the substrate.

First, it needed to be determined whether pore-spanning membranes can be spread with high surface coverage, and whether the membrane protrusions could be investigated further. For this purpose, the spreading process was investigated in more detail.

For this, silica substrate was soaked in buffer solution ( $1 \mathrm{mM}$ pyranine, $100 \mathrm{mM} \mathrm{KCl}, 10 \mathrm{mM}$ Tris, pH $7.4 \quad 0.214$ Osmol/kg). Sucrose filled POPC GUVs labeled with $0.5 \%$ Texas Red DHPE were spread on silica pore substrate. During the spreading process, only the Texas Red DHPE fluorescence was detected, since the excess pyranine in the supernatant buffer solution made detection of pyranine within the pores impossible.

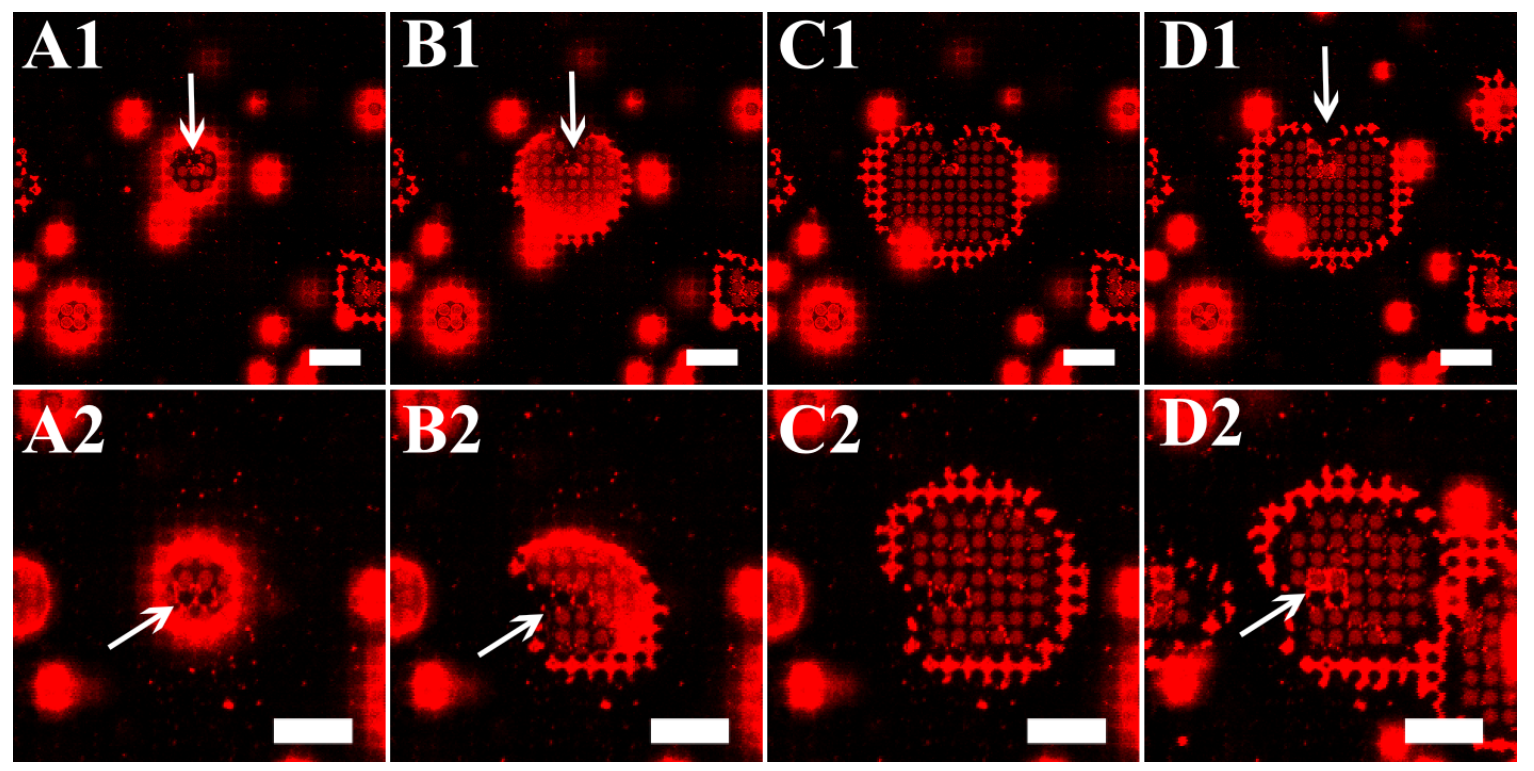

Fig. 3.3: Confocal laser scanning micrographs of two Texas Red DHPE doped giant unilamellar vesicles (top and bottom panels) during spreading process onto a closed silica pore substrate. Two time series (A-D1 and A-D2) of spreading vesicles are shown. Images were taken in the confocal plane of the substrate in $30 \mathrm{~s}$ intervals. Arrows: Possible tear origin of vesicle rupture. Scale bar: $20 \mu \mathrm{m}$. 
As Fig. 3.3 illustrates, the obtained fluorescence images allowed for a documentation of the process of GUV spreading. Two individual vesicle spreading events are depicted (upper and lower panels). The vesicles are seen to approach the membrane and come in to the plane of focus (images A).

Vesicle contact with the surface can be seen by the red fluorescence of the pores which encompasses about $4-9$ pores, depending on the vesicle size. The vesicles remained stable on the surface for few minutes until spontaneous spreading occured. The spreading process started after about $30 \mathrm{~s}$ to 5 minutes.

The spreading occured in the characteristic manner, where a heart-shaped membrane was deposited on the pore (images B, C, and D). The characteristic kink in shape of the resulting planar membrane probably arose from when the GUV ripped open, and its curved membrane folded onto the porous substrate to form a planar membrane. The brightly fluorescent ring structures in the periphery of the formed planar membrane probably represent multilamellar lipid structures which form as artifacts on the edges of the ripped vesicle membrane. Further experiments thus concentrated on reactions occurring in the darker center of the membrane patches, and disregarded the periphery.

\subsubsection{Membrane behavior and stability after GUV spreading}

After successful spreading, supernatant buffer was exchanged and it was tested whether pyranine-containing buffer remained trapped in the cavities below the membrane patches. The morphological changes in the membranes over time were investigated.

Fig. 3.4 shows the morphological changes of a membrane patch during (A) and after spreading (B), and after the addition of a low (C) and high (D) concentration of melittin. In the first set of images, the vesicle is seen to touch the substrate surface and spread, forming a planar membrane. A fluorescing membrane patch with underlying hexagonal patterns from the support is visible. In Fig. 3.4 B1 - B3, many small ring structures at the pore rims are visible, which appear to be due to the membrane protrusions, as seen in the cross sections depicted in B (top and right image edges). It appears that the pore-spanning membrane sections formed spherical protrusions, which grew with time. After exchange of the supernatant buffer to buffer without pyranine, the remaining pyranine-containing buffer trapped in the cavities was imaged, and fluorescence changes upon addition of $100 \mathrm{nM}$ melittin were followed (Fig. 3.4 C). Neighboring membranes had detached from their supports and merged to larger membrane bubbles protruding from the support. Accordingly, pyranine fluorescence in the large reservoirs appeared higher than in the small reservoirs. Melittin addition led to the release of pyranine from the cavities. Here, the pyranine fluorescence vanished faster from small reservoirs than from large ones.Addition of $600 \mathrm{nM}$ melittin initially led to a shrinking of the large membrane protrusions. 

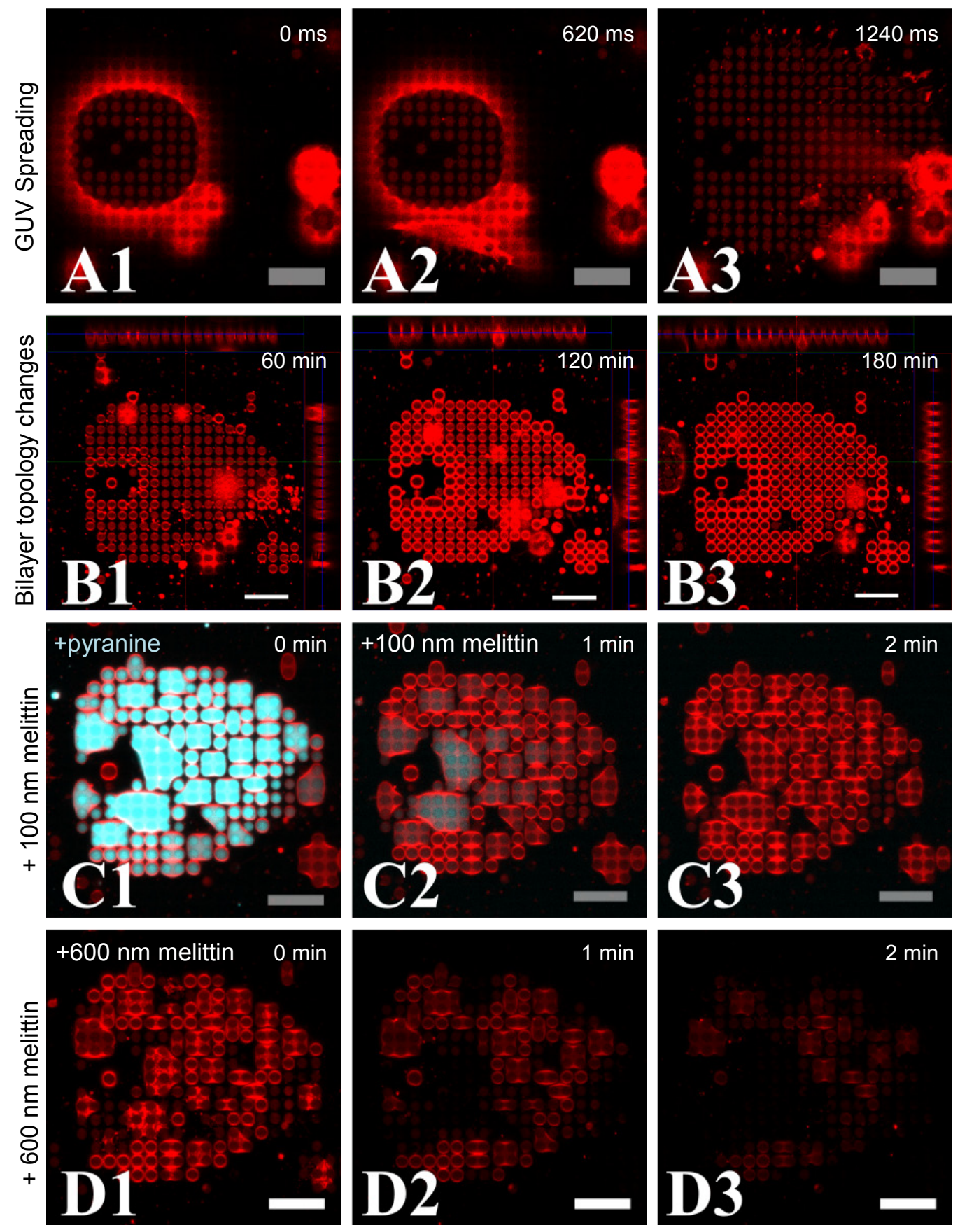

Fig. 3.4: Morphology changes of lipid bilayer patches on closed silica pores over time. GUVs were spread on silica pore substrate. Substrate had previously been soaked with buffer containing $1 \mathrm{mM}$ pyranine. Images show sample changes in chronological order from left to right and top to bottom. (A1 - A3) Spreading of GUV to lipid bilayer patch. Only Texas Red fluorescence is displayed, since during spreading the supernatant buffer contained excess pyranine. Images were acquired in $0.62 \mathrm{~s}$ intervals. (B1 - B3) Changes in lipid bilayer topology over time. Z-stack images of Texas Red fluorescence 1,2 and 3 hours after spreading, with corresponding $z$-lines at the top and right edge of each image. Free membranes between the supports started to protrude. (C1 - C3) Texas Red fluorescence (red) of the membrane and pyranine fluorescence (cyan) of the underlying dye-filled cavities after exchange of supernatant buffer. $\mathrm{C} 1$ was acquired about 200 min after A1, time specifications indicate time elapsed after pyranine addition. (C1) Pyranine-containing buffer was visibly trapped below the membrane patches. Certain parts of the membrane appeared to have detached from their support and merged to large compartments. (C2 - C3) Addition of $100 \mathrm{nM}$ melittin led to fluorescence decrease of pyranine fluorescence in the cavities. (D1 - D3) Texas Red fluorescence of the same patch after increase of melittin concentration to $600 \mathrm{nM}$. Membrane fluorescence decreased and disappeared partially. Time specifications indicate time elapsed after addition of $600 \mathrm{~nm}$ melittin. Scale bar: $20 \mu \mathrm{m}$. 
Fluorescence intensity of the Texas Red- labeled lipid bilayer deceased, and finally black holes appeared in the lipid bilayer, marking the rupture of the membrane over individual pores.

To summarize, it was shown that aqueous dye could be trapped underneath spread membranes, and that the permeabilization by melittin could be followed through dye release and changes in membrane curvature. However, the formation of spherical membrane perturbations after spreading was an unintended effect. The behavior of the system was checked in a more detailed analysis, to gain better understanding of this effect.

\subsubsection{Detailed assessment of dye release on closed silica pores}

The experiment was repeated to characterize the changes of pore-spanning membranes on closed silica support more closely. For this, silica substrate was soaked in buffer solution ( $1 \mathrm{mM}$ pyranine, $100 \mathrm{mM} \mathrm{KCl,} 10 \mathrm{mM}$ Tris, $\mathrm{pH} 7.4 \quad 0.214$ Osmol $/ \mathrm{kg}$ ). Sucrose filled POPC GUVs labeled with $0.5 \%$ Texas Red DHPE were spread on silica pore substrate. During the following rinsing procedure, excess pyranine was removed and only the dye trapped beneath the newly formed pore-spanning membranes remained. Two-dimensional confocal laser scanning images were taken in a time-lapse setting in 1 min intervals.

Fig. 3.5 gives a more detailed view of the effects of melittin on membrane (Texas Red) and pyranine fluorescence. The addition of $100 \mathrm{nM}$ melittin caused the release of pyranine from the pores, while the membrane layer remained intact. These data show that the membranes became indeed permeable to pyranine through melittin. Also in this experiment, red ringstructures of the membrane suggested that membrane protrusions were forming. It was

considered that osmolarity differences between the buffers in and outside the cavities caused these perturbations. As this may cause membrane stress, it was questioned whether the existing setup would be suitable to monitor further membrane perturbations through permabilizing agents with enough precision. Furthermore, the cavities of the substrate only hold a small reservoir of pyranine, which caused problems of photobleaching throughout the experiment.

To summarize, it was shown that pore-spanning membranes on closed silica pores are in principle suitable for membrane permeabilization experiments through a dye release assay. However, during the course of experiments it became clear that the caveats of the approach, such as the necessity to apply gold coating for functionalization, unintended membrane bulging and photobleaching problems during dye release, made the system too unreliable for consistent data acquisition. In an approach to overcome the aforementioned problems, an alternative porous substrate with different surface functionalization was investigated, and a dye inflow assay was tested as an alternative to a dye release assay. 

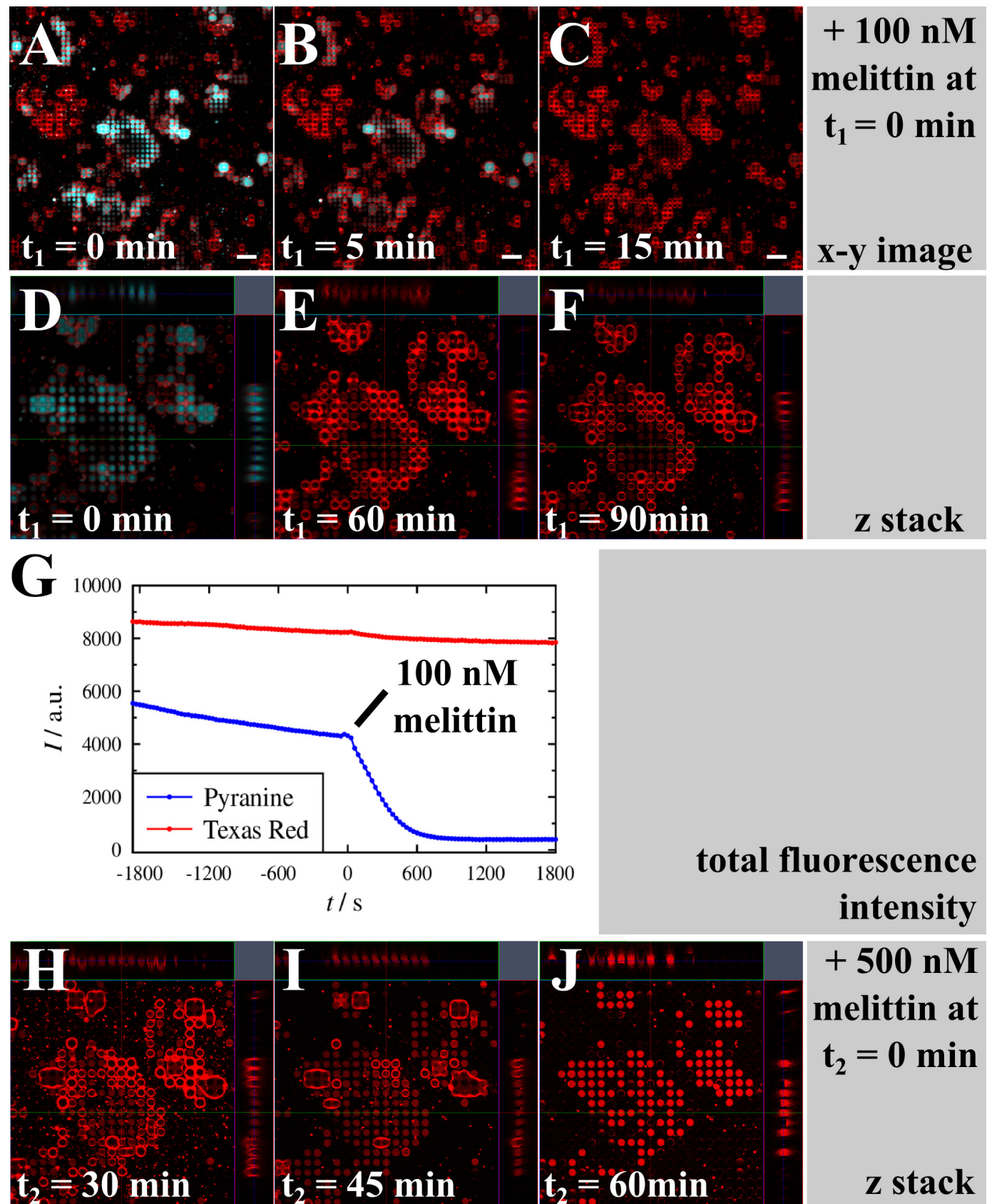

Fig. 3.5: Time-lapse of confocal laser scanning micrographs of Texas Red DHPE doped pore-spanning membrane (red) with trapped pyranine (cyan) underneath the membrane patches. 2D images (A-C) and zoomed in $\mathrm{z}$-stacks of the images (D-F) show the effects of $100 \mathrm{nM}$ melittin at $\mathrm{t}=0 \mathrm{~min}$ on membrane and trapped buffer reservoir. Melittin addition led to a decrease of pyranine fluorescence beneath the membrane patches, and to a mild decrease in membrane fluorescence. (G) Time course of the fluorescence changes of the membrane (Texas Red) and aqueous dye in the substrate pores (pyranine) of the samples shown in A-F. (H-J) Z-stack of the same patch after addition of $500 \mathrm{nM}$ melittin. Scale bar $20 \mu \mathrm{m}$. 


\subsection{Pore-spanning membranes on AAO support: Investigation of AMPs}

In the latter part of this work, optically transparent anodic aluminium oxide (AAO) was used as a support for pore-spanning lipid bilayers (for preliminary work see [94],[97]). The optical transparency allows for the detection of fluorescent dyes in the cavities, as the dye is excluded from the cavities by the lipid bilayer patches. The optical transparency allows it to set the optical focus deeper into the cavities, so that the fluorescence changes in the cavities can be detected during a dye inflow assay.

The AAO can be hydrophilically functionalized by selective silanization, and no further gold application is mandatory. Beyond the minimization of photo bleaching effects, the optical transparency of the AAO substrate allows for a detailed observation of the lipid bilayer topology and morphology that is limited on the gold-supported silica pores because of quenching effects. AAO pores have a diameter of $70 \mathrm{~nm}$. This provides a tighter support mesh for the suspended lipid membrane.

To assess whether pore-spanning lipid bilayers are a suitable model systems for the investigation of antimicrobial peptides, typical characteristics of the used lipid bilayer had to be determined. In this chapter, qualitative characteristics of the lipid bilayer before and after treatment with permeabilizing agents were investigated.

Substrates were prepared with pore-spanning membranes according to 3.2.1. Instead of trapping an aqueous dye in the cavities, it was added to the supernatant buffer, and influx was monitored. While this method would in principle be applicable for silicon pores as well, quenching through gold sputtering and the difficulty of the microscope to focus deep into the pores made the silicon substrate unsuitable for this approach. Prior to addition of antimicrobial peptides, two- and three-dimensional confocal laser scanning microscopy images of typical lipid bilayer patches were taken. The basic parameters assessed were the initial fluorescence intensity of the Texas Red DHPE-labeled lipid bilayer, and the fluorescence of the aqueous fluorescence dye in solution. Accordingly, the change of fluorescence in the AAO cavities under the lipid bilayer upon peptide addition was observed.

Since the lipid bilayer topology was considered an important readout parameter, the typical topology before permeabilization had to be investigated in more detail. This was done by using labeled lipids.

Finally, permeabilizing agents were added to the lipid bilayer and the change in membrane topology and the course of fluorescent dye inflow into the cavities were investigated.

The next chapter will deal with the initial characterization of the system, using an exemplary image from experiments discussed in chapter 3.4.1. 


\subsubsection{General topology of the lipid bilayer patches}

The typical topology of a lipid bilayer patch after spreading and the characteristics of dye influx into the cavities of the AAO support were investigated.

POPC-giant unilamellar vesicles (GUVs) doped with 0.5 mol\% Texas RED DHPE were spread onto closed AAO pores with a diameter of ca. $70 \mathrm{~nm}$. After spreading, the buffer volume was exchanged to remove residual GUVs and lipid material. Pyranine was added to the buffer environment to a final concentration of $1 \mathrm{mM}$. The lipid bilayers were located uder the microscope using a water immersion objective $(63 \mathrm{x})$ and the widefield function of a confocal laser scanning microscope. A patch with homogeneous shape was selected. Confocal laser scanning microscope images of the Texas RED DHPE and pyranine fluorescence were taken. Images of the membrane were taken in the confocal plane of the lipid bilayer. Images of the cavity content were taken in a confocal plane under the lipid bilayer. Additionally, a zstack image was generated to observe the three dimensional topology of the patch and the pyranine distribution in the cavities.
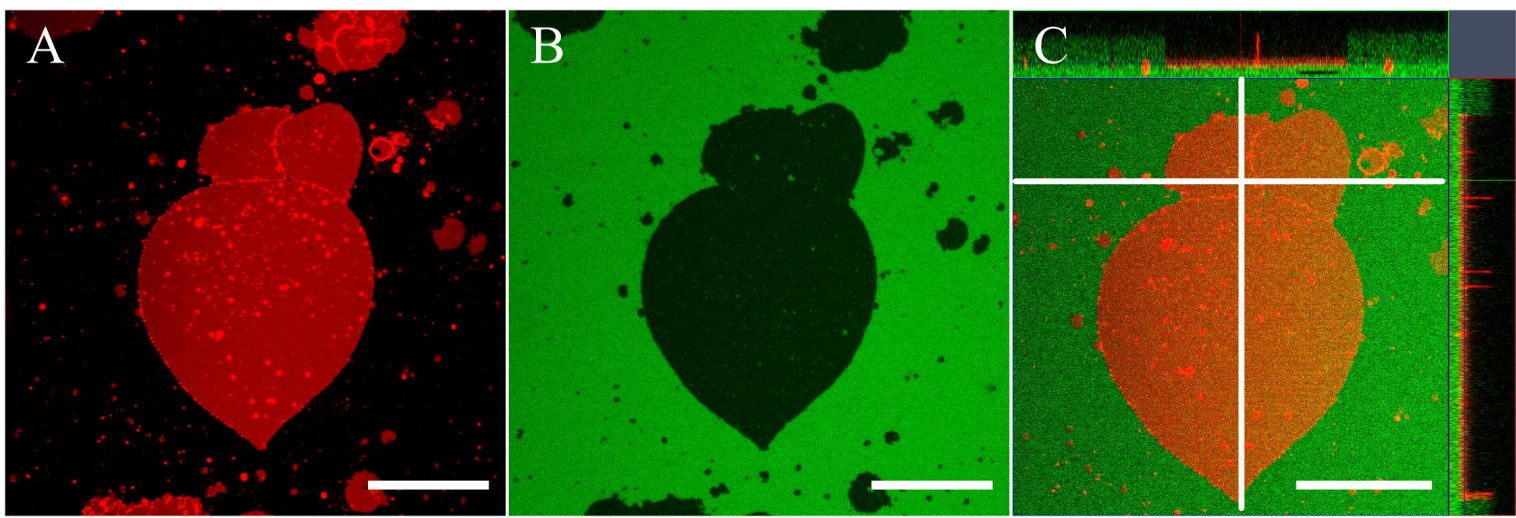

Fig. 3.6: Representative confocal laser scanning micrographs of Texas Red DHPE (red) labeled lipid bilayer patches on functionalized AAO with pyranine (green) in supernatant buffer solution. (A) Texas Red DHPE (red) fluorescence in the focal plane of the lipid bilayer patch. Large main patches are surrounded by smaller patches and lipid material, visible as red dots. Bright lines and dots represent excess lipid material from the spreading process that is either located on the substrate or in the substrate cavities. (B) Image of the corresponding pyranine fluorescence (green) in the focal plane of AAO cavities under the lipid bilayer patches. Fluorescence is detected in cavities which are not sealed by pore-spanning membranes. Meanwhile, non-filled cavities appear black. Green dots correspond to small defects in the lipid bilayer that allow pyranine to diffuse in the underlying cavities. (C) Orthogonal view of a z-stack image with an overlay of Texas Red and pyranine fluorescence. White lines: z-line cross sections of the patch. The corresponding optical cross sections are shown at the top and right of the image. Scale bars: $20 \mu \mathrm{m}$.

As seen in Fig. 3.6A and C, large lipid bilayer patches with diameters of $>20 \mu \mathrm{m}$ and relatively homogeneous fluorescence could be obtained. Membrane patches were often heartshaped, and generally had a diameter between 1-40 $\mu \mathrm{m}$. In the corresponding Fig. 3.6B, pyranine fluorescence (green) is detected in the focal plane of the AAO cavities, while unfilled cavities appear black. The non-fluorescent areas directly correspond to the areas that are sealed by the lipid bilayer. The figure also shows that the spreading of GUVs in close 
proximity to each other led to adjacent individual patches rather than a large homogeneous membrane. These patches appeared to be separated by bulk membrane material which caused bright lines on the patch edges. In an orthogonal view representation of a z-stacked image, these lines could be identified as lipid material that has penetrated into the AAO cavities and seemed to coat the entire cavities. In a cross section image, this lipid material is visible as perpendicular red lines ("spikes") that begin from the lipid bilayer plane and end at the glass support. These red lines often appeared at the edges of a large lipid bilayer patch. During the spreading process, deposition of smaller GUVs and residual lipid material could not be fully avoided despite intensive rinsing with buffer solution. This led to smaller red patches of about $<1 \mu \mathrm{m}$ size with corresponding unfilled AAO cavities. Much like lipid bilayer patches, small vesicles were also able to seal cavities or adhere to the surface of lipid bilayer patches. These adhered vesicles appeared as small bright red dots. Additionally, lipid material that had entered the cavities could appear as red dots in cross sections of the Texas Red fluorescence. The latter could only be distinguished from vesicles by three-dimensional imaging.

Experimental experience showed that, generally, lipid bilayer patches were stable and impermeable for fluorescent dyes for at least one day (data now shown). However, small defects in the membrane could occur during the spreading process, leading to individual pyranine filled pores. These were recognizable as black dots in the red lipid bilayer fluorescence, and corresponding green dots in $2 \mathrm{D}$, or perpendicular lines in $3 \mathrm{D}$ pyranine fluorescence representation. These defects seemed to appear directly during the spreading process, since the number of defects was stable over hours and did not increase. Moreover, damage of the membrane during the rinsing process was observed, which led to open cavities that appeared black in red and green fluorescence (data not shown).

To avoid false-positive permeabilization readouts due to the use of initially damaged lipid bilayers, only bilayers with very homogeneous topology were chosen for further experiments.

The primary quality control of the lipid bilayers was absolutely critical for any consecutive experiments, and was carried out diligently.

To summarize, the properties of the lipid bilayers were studied in detail. This was critical for understanding the observed signal patterns. The careful observation of occurring fluorescence signals led to the definition of parameters for the quality control of all following lipid bilayer preparations. Optical properties of optimal lipid bilayers were defined, and enabled the identification of areas of interest in the following experiments. 


\subsubsection{Determination of a suitable data acquisition mode}

Ideally, a reconstructed 3D image of the lipid bilayer on the porous substrate, as well as the underlying cavities, would yield the most complete information on system changes during the experiment. In this case, the fluorescence under the lipid bilayer could be detected and followed in a time dependent manner. However, time resolution is limited by the scanning speed of the used confocal laser scanning microscope setup. To acquire data with adequate time resolution, a single line in $\mathrm{x}-\mathrm{y}$ position crossing the membrane patch was recorded representatively for the whole patch, and stacked ("z-line scan"), as illustrated in Fig. 3.7.

The resulting image equals a cross-section of a $3 \mathrm{D}$ z-stack image.

Additionally, 2D images of the xy plane of the setup were acquired before and after the addition of antimicrobial peptide.

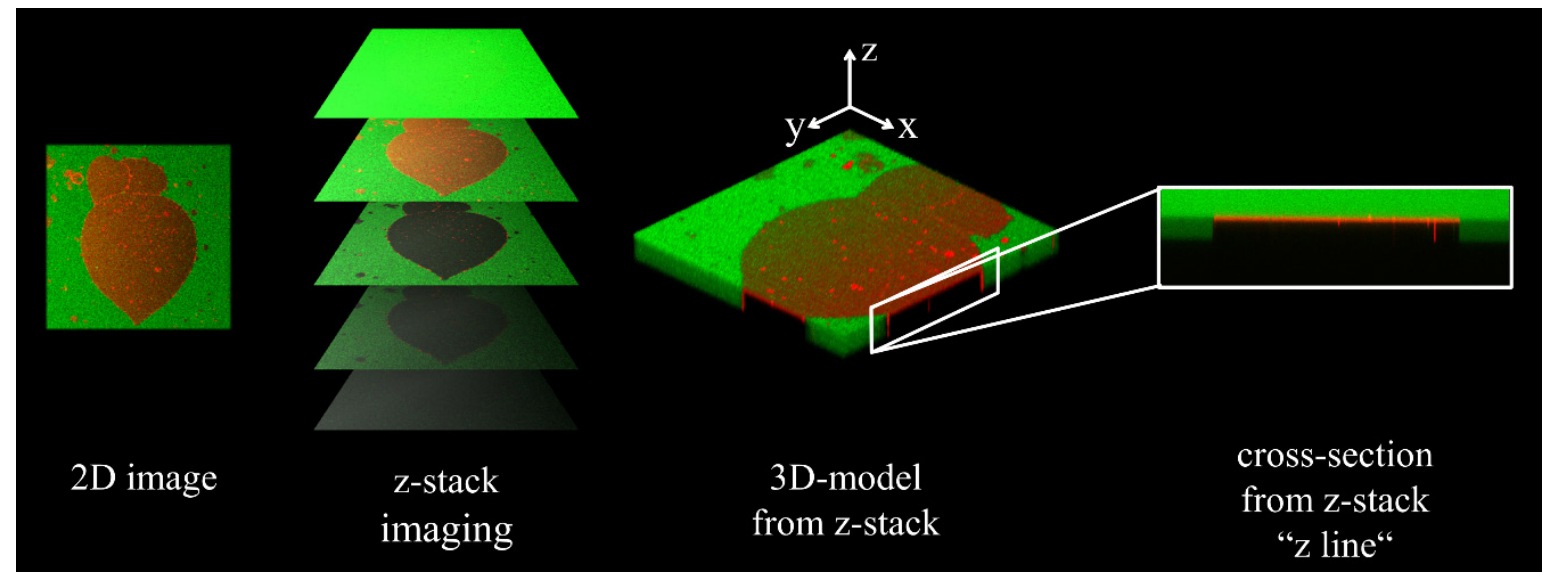

Fig. 3.7: Schematic representation of the three dimensional image acquisition: Two-dimensional images of a lipid bilayer patch are taken in xy plane. Texas Red DHPE fluorescence of the lipid bilayer (red) and fluorescence of the water-soluble dyes pyranine/FITC dextran (green) are acquired. Empty pores and glass support show no fluorescence (black). Images from consecutive z-direction focal planes can be stacked, and a 3D-image can be calculated. Alternatively, only a line crossing the lipid bilayer patch area in $\mathrm{x}-\mathrm{y}$ direction can be scanned and stacked in $\mathrm{z}$-direction for better time resolution. The resulting image ( $\mathrm{z}$ line) presents a cross section of the membrane patch.

This setup was expected to allow simultaneous monitoring of the lipid bilayer fluorescence and the fluorescent dye inflow. It was assumed that the observed changes in a cross section of the patch is representative for the whole patch. Due to the reduced image acquisition time also the effect of photobleaching could be minimized. It should be kept in mind however that the two-dimensional representation limits the possibility to observe the whole lipid bilayer patch topology at once.

In contrast to membrane model systems using vesicles, where the lipid bilayer encloses a single compartment, pore-spanning lipid bilayers feature multiple aqueous compartments on the support site. The qualitative characteristics of the model system after permeabilization had to be determined before a quantitative analysis was possible 
The data obtained with these modes of image acquisition will be addressed consecutively in the following chapters.

\subsubsection{Changes in membrane and cavity fluorescence after permeabilization: qualitative changes visible in cross sectional view}

Cross sectional imaging was used to get information in a satisfying time-resolved manner. A membrane preparation was made as described in 3.3.1. The peptide melittin, which is known for solubilizing lipid bilayers, was added at $1.4 \mu \mathrm{M}$ to the buffer solution. The changes in lipid bilayer fluorescence and pyranine $(5 \mathrm{mM})$ inflow into the cavities were monitored. Cross-sectional $\mathrm{z}$ line images were taken in an interval of $30 \mathrm{~s}$. Representative images at the given time points are shown in Fig. 3.8. An overlay of Texas Red and pyranine fluorescence is shown in (A), while (B) only shows Texas Red fluorescence to highlight the changes in membrane topology.
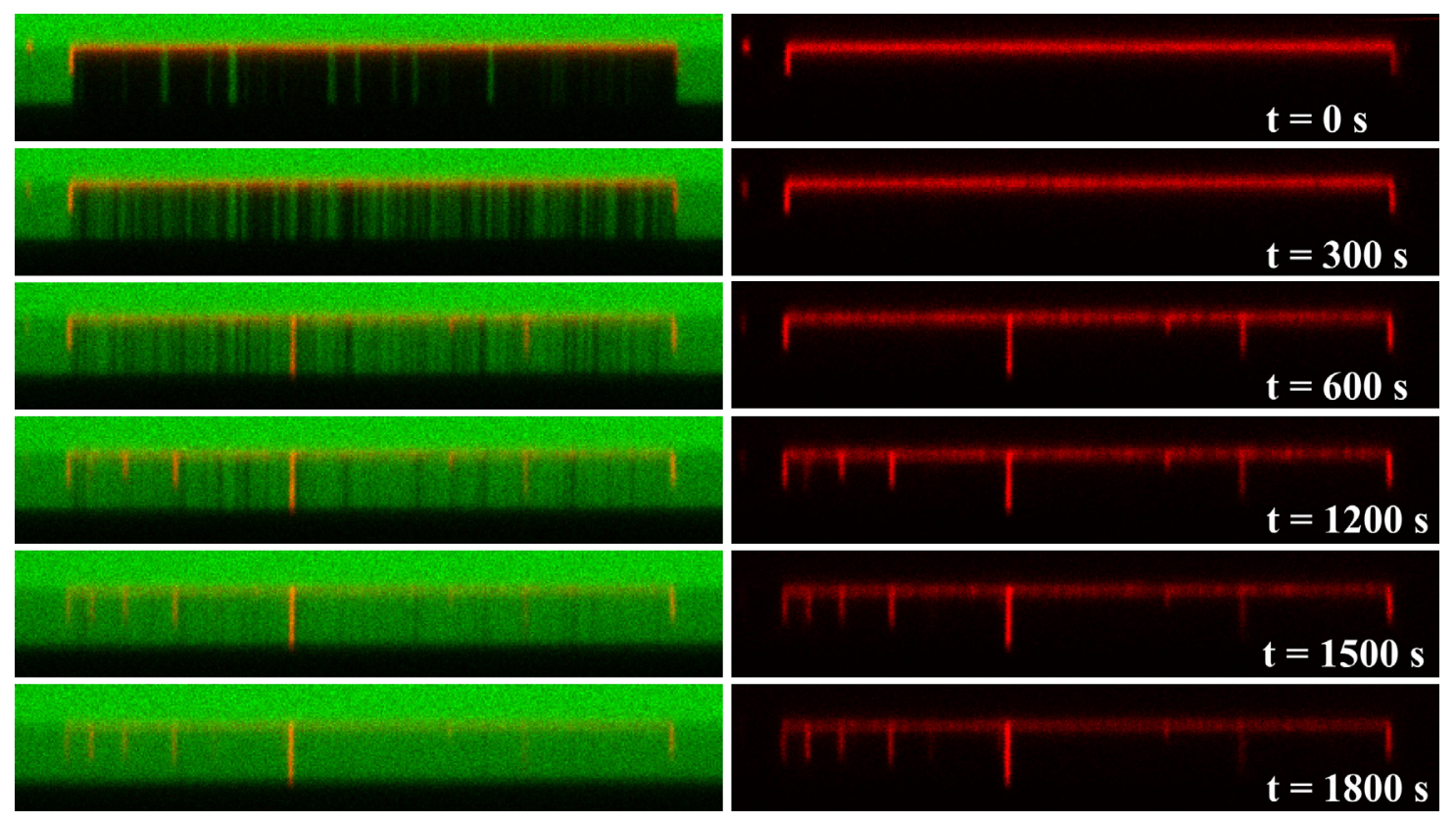

Fig. 3.8: Z-line images of pore-spanning membranes over time. Overlay of confocal laser scanning z-line fluorescence images, showing pore-spanning Texas-Red DHPE-labeled bilayer (red, left and right panels) and pyranine fluorescence (green, left panels) at the given time points after melittin addition. Pyranine is detected in the bulk phase (top), and the cavities (middle). Unsealed cavities under the pore-spanning membrane are filled with pyranine (green spikes). The AAO glass support is black (bottom). Upon addition of $100 \mathrm{nM}$ melittin, $(\mathrm{t}=0 \mathrm{~s})$, gradual filling of the cavities underneath the membrane is visible in the appearance of green spikes. The lipid bilayer shows homogeneous fluorescence before melittin addition. At $\mathrm{t}=300 \mathrm{~s}$ after melittin addition, the membrane shows changes in the form of small fluorescence gaps. Red spikes $(t=600 \mathrm{~s})$ indicate the migration of lipid material into the inside of the cavities. Further decrease of overall fluorescence intensity is seen throughout the remaining membranes.

Fig. 3.8 depicts consecutive z-line scans of a membrane patch (red) with water-soluble pyranine in the supernatant buffer (green). At the beginning $(t=0)$ of the experiment the red 
fluorescence of the lipid bilayer was distributed homogeneously over the pores. Correspondingly, the underlying cavities appeared mainly black, indicating that they were not filled with the green dye.

After melittin addition, an increase of green spikes could be observed. Each spike represents at least one or multiple adjacent cavities that are filled with fluorescent dye. After a certain lag time $(t=300 \mathrm{~s})$, the cavities started to show increased green fluorescence. Gradual filling of the cavities could be observed until complete filling of all pores, visible by a fluorescence increase to a final intensity as under open cavities. As will be addressed later, an alternative final outcome was an equilibrium state where the cavities beneath the membrane patch retained a lower fluorescence than the uncovered cavities, indicating a final state of incomplete filling.

It was also observed that the total fluorescence intensity of the lipid bilayer significantly started to decrease after melittin addition (this is an effect that is characteristic for melittin action, as will be discussed later). Small vertical gaps appeared in the lipid bilayer. After a while, red horizontal lines appeared $(t=600 \mathrm{~s})$ that ended at the barrier between AAO and glass support. They likely represent pores whose cavities coated with membrane material after rupture of the suspended lipid bilayer. These spikes remained at a relatively constant fluorescence, while the fluorescence of the lipid bilayer spanning the cavities decreased further.

Defects in the lipid bilayer fluorescence could be observed as gaps (black vertical lines). These defects could only be appreciated qualitatively since they are in the magnitude of the resolution limit of the confocal microscope.

\subsubsection{Changes in membrane and cavity fluorescence after permeabilization: qualitative changes visible in $2 \mathrm{D}$ imaging of the $\mathrm{xy}$ plane}

Line scanning allows simultaneous monitoring of the membrane topology and dye translocation over the lipid bilayer into the cavities. However, it does not provide information about the topology of the whole lipid bilayer patch.

To get further information about the change in membrane topology, two-dimensional images were taken in the xy plane. This allowed a detailed view of the lipid bilayer topology. It was not possible to simultaneously monitor the dye translocation and topology change of the whole lipid bilayer patch in a satisfying time resolution. To overcome this, images of the complete lipid bilayer patches before and after peptide addition were acquired. The latter images were taken when an equilibrium state of cavity filling had been reached.

Here, three membrane-permeabilizing agents were used to induce changes in membrane topology. The two model peptides melittin and magainin-2, as well as the detergent Triton X100 were used. The results of the $2 \mathrm{D}$ images were compared with cross sectional images. 


\subsubsection{Membrane dynamics upon melittin treatment}

Membrane preparations were made as described in 3.3.1, where POPC-lipid bilayer patches labeled with $0.5 \mathrm{~mol} \%$ Texas Red DHPE were spread onto closed AAO substrate pores. Melittin in a final concentration of $1.4 \mu \mathrm{M}$ was added. Two-dimensional images were acquired before the administration of melittin and after an equilibrium state of cavity filling (as observed through pyranine fluorescence in parallel image acquisitions in cross sectional view).

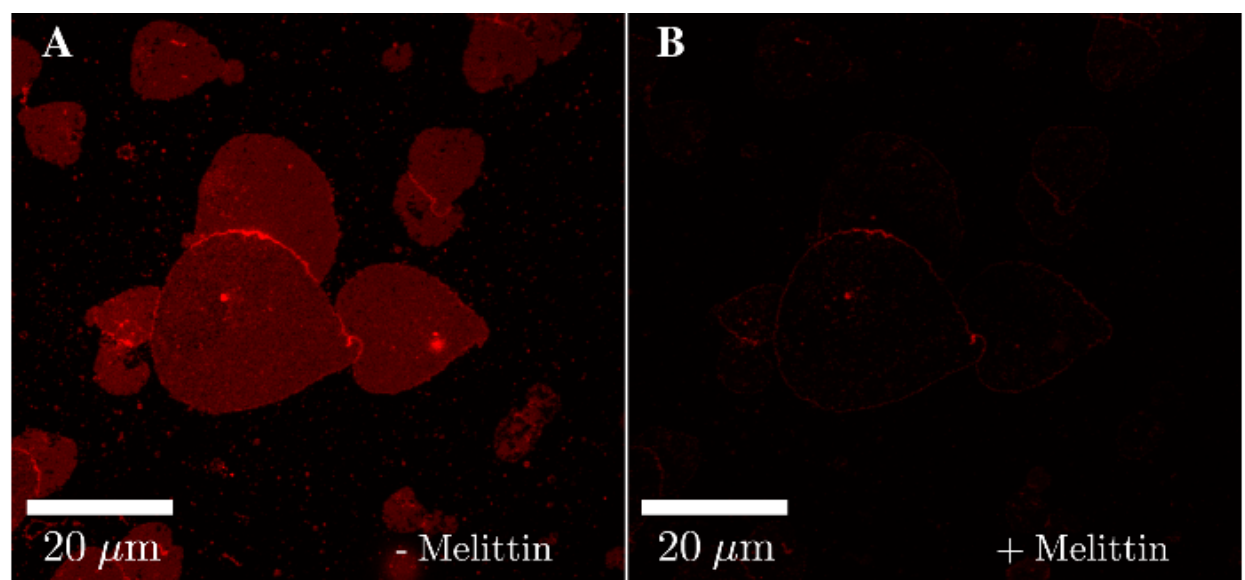

Fig. 3.9: Confocal laser scanning micrographs (top view of the porous substrate) of Texas-RedDHPE (0.5 mol\%) labeled POPC pore-spanning lipid bilayers (A) before and (B) after melittin addition in a total concentration of $1.4 \mu \mathrm{M}$. (A) Typical homogeneous fluorescence of the lipid bilayer patch is shown. (B) A significant loss of lipid bilayer fluorescence is observable. Only the contour of the membrane patch is visible after the time series experiment. Scale bars: $20 \mu \mathrm{m}$

Fig. 3.9 shows a characteristic lipid bilayer patch before and after addition of melittin. Before melittin administration, a homogeneous lipid bilayer fluorescence was visible. Brighter contours and bright spots were observed that were characterized in chapter 3.3.1 as lipid material that accumulated in the cavites after spreading. Melittin is known to lyse lipid bilayers. Accordingly, after addition of melittin, a drastic loss of homogeneous membrane intensity was observable, suggesting the complete loss of pore-spanning membranes. Only the contour of the membrane patch was visible after the time series experiment. This contour had the characteristic features of lipid material that is inside the cavities and stuck to the pore walls, as discussed in 3.3.1. Additionally, a significant decrease in total fluorescence was observed which exceeded the loss expected through photobleaching.

\subsubsection{Membrane fluorescence changes upon magainin-2 treatment}

The antimicrobial peptide maganin-2 is discussed to have a different mode of action from melittin (1.1.3). It was therefore used to test if the assay could reflect any differences in the mode of action in the fluorescence output. Membrane preparations were made as described in 3.3.1, where POPC lipid bilayer patches labeled with 0.5 mol\% Texas Red DHPE and spread 
onto closed AAO substrate pores. Magainin-2 in a final concentration of $6.6 \mu \mathrm{M}$ was added to the supernatant buffer. Two-dimensional images were acquired before the administration of magainin-2 and after an equilibrium state of cavity filling (as observed through pyranine fluorescence in parallel image acquisitions in cross sectional view).

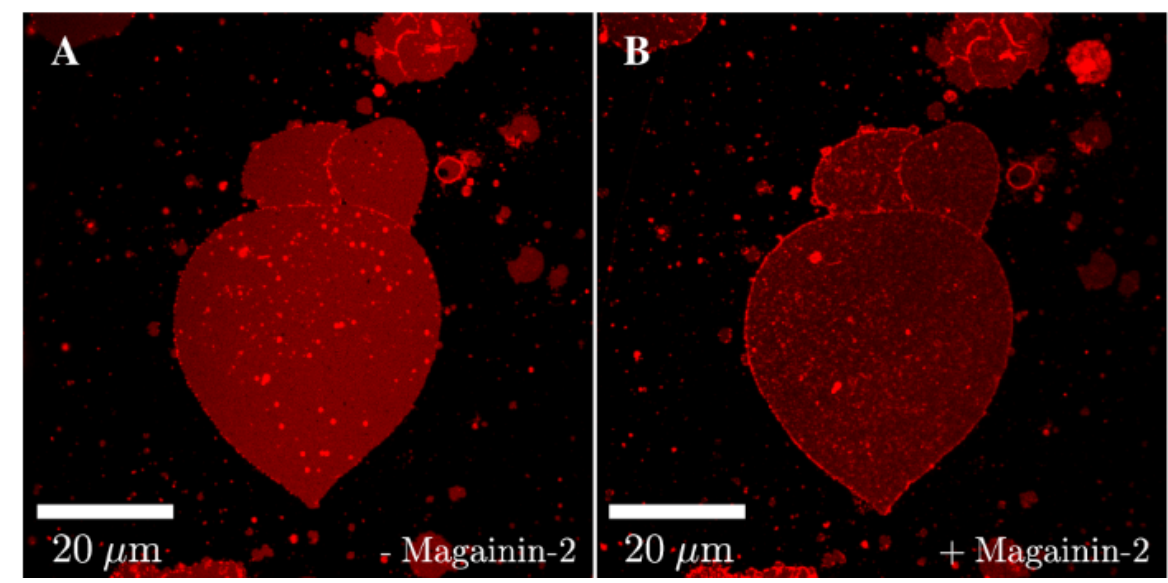

Fig. 3.10: Confocal laser scanning micrographs (top view of the porous substrate) of Texas-Red-DHPE (0.5 mol\%) labeled POPC pore-spanning lipid bilayers (A) before and (B) after magainin-2 addition in a final concentration of $6.6 \mu$ M. Membrane fluorescence was acquired in the same focal plane. (A) Typical homogeneous fluorescence of the lipid bilayer patch is shown. (B) A slight decrease of fluorescence intensity is noticeable, but the homogeneous fluorescence of the lipid bilayer can still be observable after the cavities are completely filled. Scale bars: $20 \mu \mathrm{m}$

As can be seen from Fig. 3.10, the lipid bilayer patch showed a homogeneous fluorescence before addition of the antimicrobial peptide magainin-2. Brighter fluorescence spots result from lipid material accumulated in the cavities, as discussed in chapter3.3.1. In contrast to melittin (Fig. 3.9) the homogeneous fluorescence of the lipid bilayer after magainin-2 addition was preserved aside from a slight but distinct decrease in fluorescence intensity. This corresponds to the established mode of action of magainin-2 (1.1.3), which is the formation of toroidal pores without the removal of lipid material from the membrane.

\subsubsection{Membrane fluorescence changes upon Triton X-100 treatment}

To obtain data on the strongest possible fluorescence changes in the used setup, complete membrane removal was induced by the addition of the detergent Triton X-100. Membrane preparations were made as described in 3.3.1, where POPC lipid bilayer patches labeled with $0.5 \mathrm{~mol} \%$ Texas Red DHPE were spread onto closed AAO substrate pores with a diameter of $70 \mathrm{~nm}$. Triton X-100 in a final concentration of $0.5 \mu \mathrm{M}$ was added to the supernatant buffer. Two-dimensional images were acquired before the administration of Triton X-100 and after an equilibrium state of cavity filling (as observed through pyranine fluorescence in parallel image acquisitions in cross sectional view) was reached. 

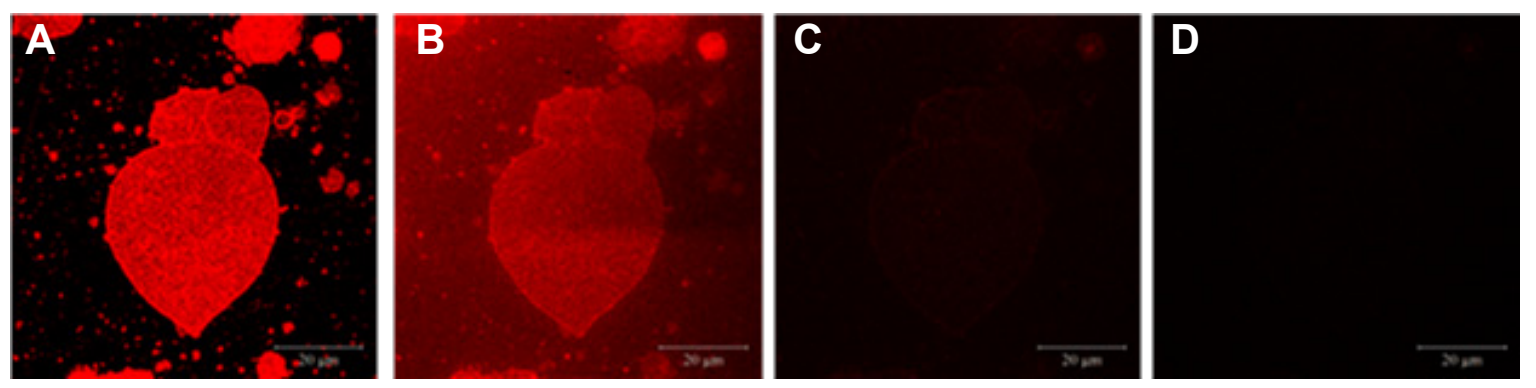

Fig. 3.11: Confocal laser scanning micrographs (top view of the porous substrate) of Texas-Red-DHPE (0.5 mol\%) labeled POPC pore-spanning lipid bilayers (A) before and (B-D) after the addition of $0.5 \mu \mathrm{M}$ Triton X-100. After administration of Triton-X 100, a decrease of red fluorescence in the focal plane is observed, while the background fluorescence initially increases. The membrane fluorescence then completely disappears. Scale bar $20 \mu \mathrm{m}$.

Triton X-100 addition led to a decrease of membrane fluorescence intensity, accompanied by an initial increase in background fluorescence (Fig. 3.11). This suggests that lipid material was removed from the membrane by the detergent and starts to diffuse, diluting the fluorescing particles in the bulk supernatant. This is why background fluorescence initially increased, and later decreased. In contrast to magainin-2 and melittin, the Texas Red fluorescence notably disappeared in the AAO cavities as well.

\subsubsection{Comparison of imaging results}

The presented data show that characteristic, differentiable fluorescence signals can be obtained from lipid bilayer patches and from lipid material that is located in the cavities. In the xy plane, a lipid bilayer patch shows a homogeneous fluorescence directly after spreading. Lipid material that is located in the cavities is observed as small dots, lines are areas of significantly higher fluorescence intensity. In cross sectional view, the latter form spikes protruding from the membrane bilayer onto the bottom of the pores.

The most significant change in lipid bilayer topology that can be observed after addition of a permeabilizing agent is the disappearance of the homogeneous lipid bilayer patch. This can be a decrease in fluorescence intensity that can result in a complete loss of fluorescence. Typically, lipid material inside the cavities retains stable fluorescence intensities or increases fluorescence during the permeabilization process.

To sum up, the 2D imaging allowed for a differentiation of modes of action when comparing melittin, magainin-2 and Triton X-100 effects. The characteristic changes in fluorescence intensity and localization suggest different interaction modes of the tested substances. Melittin appears to disturb the lipid bilayer. It affects, but does not dissolve, the lipid membrane. Meanwhile, magainin-2 leaves the lipid bilayer intact. Triton X-100 dissolves membranes regardless of whether they are a lipid bilayer or accumulated aggregates. 
While these observations gave first insights in the different modes of action of permeabilizing agents, it was aimed to gain better understanding of the underlying processes through a quantitative image analysis of the kinetic data.

\subsubsection{Quantitative analysis of fluorescence changes upon membrane permeabilization}

It was necessary to restrict the image acquisition to time-resolved z-lines (cross sections) of the sample because of long acquisition times of several minutes per image in the confocal laser scanning microscope setup. It was assumed that the observations in a cross section of the patch are representative for the whole patch. Additionally, the state of the membrane patches before and after AMP administration was documented with single 2D images in xy plane.

The acquisition of a single $\mathrm{z}$-line required the development of a way to compensate for focus drift.

\subsubsection{Visual representation and analysis of fluorescence intensity changes}

The time course of dye inflow was observed as change in fluorescence intensity $\left[\mathrm{I}_{\text {ROI }}(\mathrm{t})\right]$ in a region of interest (ROI) under the membrane patch. Additionally, the intensity of a ROI in non-sealed cavities $\left[\mathrm{I}_{\text {ref }}(\mathrm{t})\right]$ was set as a reference for complete pore filling (see Fig. 3.12). The relative fluorescence intensity $\left[\mathrm{I}_{\text {rel }}(\mathrm{t})\right]$ as a function of time $t$ was calculated by normalizing $I_{\text {ROI }}(t)$ by the fluorescence intensity at $t=0\left(I_{0}\right)$ and standardizing against $I_{\text {ref }}(t)$, a Boltzmann function was fitted to the data to analyze the time course of the resulting sigmoidal curves, as described in 2.4.4.

Fig. 3.12 illustrates with a representative data set how center time $\left(t_{1 / 2}\right)$, tangent slope $(\tau)$, and maximum fluorescence intensity $\left(\mathrm{I}_{\mathrm{MAX}}\right.$ ) as values for the time point of $50 \%$ pore filling, filling speed and maximum filling degree were obtained on a representative dataset (see 2.4.4.). For the acquisition of the dataset, pore-spanning membranes were prepared as described in 3.3.1. 6.6 $\mu \mathrm{M}$ magainin-2 was added, and fluorescence changes were analyzed on z-line images. 
A

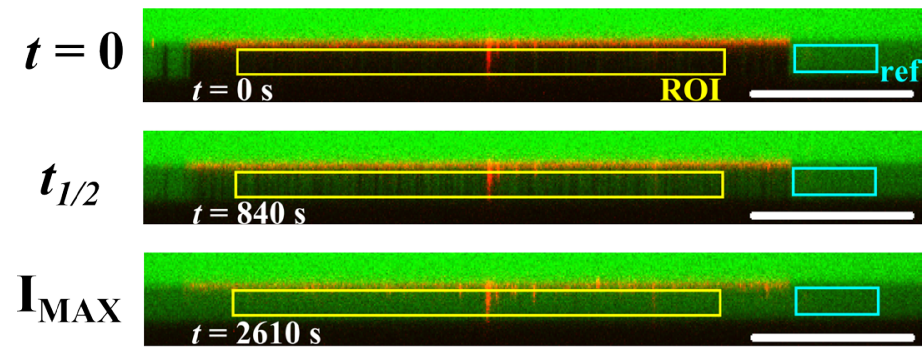

B

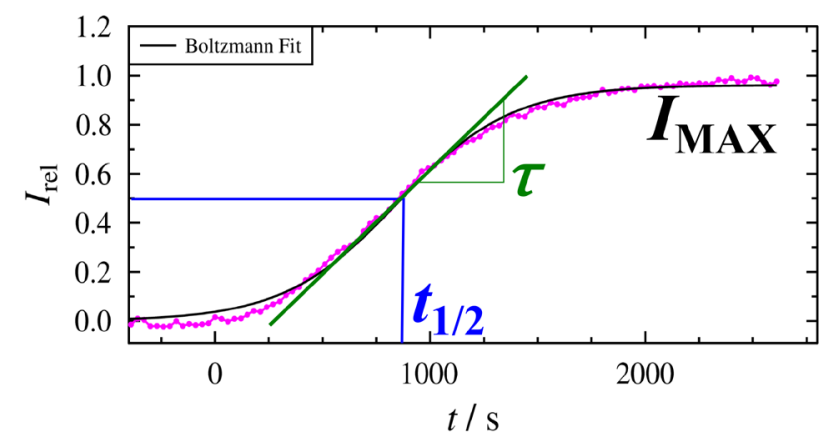

Fig. 3.12: Measurement of membrane changes over time during AMP administration. (A) Overlay of exemplary fluorescence images showing $z$-line profiles of pore-spanning Texas Red DHPE labeled POPC bilayers (red) on AAO substrate and $1 \mathrm{mM}$ pyranine (green) in bulk phase at the given time points. Images show the lipid bilayer state before addition of $6.6 \mu \mathrm{M}$ magainin-2 $(t=0)$, at the time where the fluorescence intensity reaches half its maximum value (half time $t_{1 / 2}$ ), and in the equilibrium state, where the maximum fluorescence intensity in the pores is reached $\left(I_{M A X}\right)$. The large yellow box represents the region of interest (ROI) used for the time-resolved quantitative analysis of the pyranine dye entrance by measuring fluorescence intensity, $I_{\mathrm{ROI}}$. The turquoise box presents the reference ROI, where $I_{\text {ref }}$ is measured. Upon addition of magainin-2, pyranine fluorescence (green) becomes visible in the AAO cavities that were prior sealed by the pore-spanning membrane (red). Scale bar $20 \mu \mathrm{m}$. (B) Graphic representation of the time course of measured $I_{\text {rel }}$ (pink) as a function of time, with Boltzmann fit (black). The graphic representation allows for determination of half time $t_{1 / 2}$, tangent slope $\tau$ of the sigmoidal curve at the inflection point, and maximum intensity $I_{\mathrm{MAX}}$ at the equilibrium state at the end of the experiment.

Especially in the initial phases after AMP addition, dye inflow occurred in the form of filling of individual cavities, seen as vertical green spikes below the membrane. The resolution limit of optical microscopy did not allow for "spike counting", that is, the quantification of individually filling cavities over time. For this reason, the overall cavity fluorescence was measured.

For this purpose, a MATLAB script was used that allowed to quantify relative fluorescence changes in the cavities. It was also necessary to compensate for $\mathrm{z}$-drift of the image focus during the time course of the experiments. The script was developed by Dr. Ingo P. Mey, who generously provided it for the use in this work.

In short, the script uses the data obtained from z-lines of time-lapse experiments to identify the phase boundary between AAO substrate and supernatant buffer. This phase boundary was aligned in all individual images of the dataset. The area below the membrane patch was marked by a region of interest (ROI, Fig. 3.12 top, yellow box), and time-resolved 
quantitative fluorescence values were obtained yielding the ROI intensity $I_{R O I}$ over time. In a reference measurement (ref, Fig. 3.12 top, turquoise box), $I_{\text {ref }}$ was obtained and used for normalization of $I_{R O I}$.

The changes of the obtained fluorescence intensity values depend on AMP concentration, and can be used to determine the speed and degree of filling at the end point of the experiment. This information allows for conclusions on the modes of membrane permeabilization of different agents. The MATLAB script as well as a visual example of the dataset alignment are available in the appendix.

With these measures, it was possible to visualize and quantitatively compare kinetics of dye inflow under different membrane permeabilization conditions.

In a control experiment, it will be shown that the quantitation and graphic representation could be successfully implemented.

\subsubsection{Proof of principle: Quantitation of lipid bilayer lysis with Triton X-100}

To obtain a reference measurement on complete lysis of the lipid bilayer on porous substrate support, the membrane patches were treated with $0.5 \mu \mathrm{M}$ Triton X-100. This detergent efficiently solubilizes lipid membranes through micellation of the lipids.

For this, a membrane preparation was made as described in 3.3.1, where POPC GUVs labeled with $0.5 \mathrm{~mol} \%$ Texas Red DHPE were spread onto closed AAO pores. Triton X-100 in a final concentration of $0.5 \mu \mathrm{M}$ was added to the supernatant buffer. Three water-soluble dyes were added to the bulk volume and analyzed to monitor membrane permeability: FITC-labeled dextrans $(40 \mathrm{kDa}$ and $70 \mathrm{kDa})$ at a final concentration of $5 \mu \mathrm{M}$, as well as pyranine at a final concentration of $1 \mathrm{mM}$. 


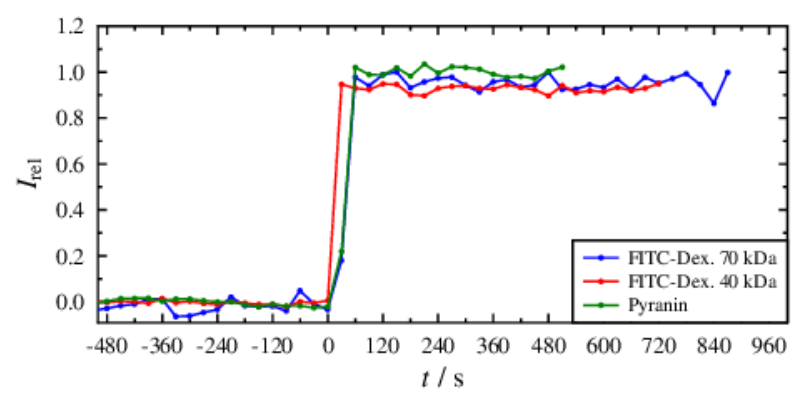

Fig. 3.13: Time course of aqueous dye fluorescence intensity Irel in AAO cavities before and after the addition of Triton X-100. A pore-spanning membrane composed of POPC doped with $0.5 \mathrm{~mol} \% \mathrm{TexasRed}$ DHPE was prepared on an AAO substrate. Pyranine (Pyr, $1 \mathrm{mM})$ or FITC-labeled dextrans with a molecular mass of $40 \mathrm{kDa}$ or70 $\mathrm{kDa}(\operatorname{Dex} 40 / \operatorname{Dex} 70,5 \mu \mathrm{M}$ ) were added to the aqueous phase and the entrance of the dyes into the cavities was read out from z-line profiles obtained by confocal laser scanning fluorescence microscopy. Time of Triton X-100 addition was set to $t=0 \mathrm{~s}$. Upon their addition, fluorescence of all dyes rises instantaneously to maximum relative fluorescence. Measurements were acquired from z-line images with MATLAB image alignment, ROI analysis and normalization against reference region. Initial and maximum fluorescence values were set to 0 and 1 , respectively.

Fig. 3.13 depicts the measured fluorescence intensities of the three dyes before and after Triton X-100 addition. An immediate increase in fluorescence inside the substrate pores was observed upon Triton X-100 addition. This increase was observed independently of the used dye. These measurements provided the values for the maximum fluorescence values with these experiment parameters. The obtained relative fluorescence values were standardized to 0 (initial fluorescence) and 1 (maximum fluorescence). They will be used later in this work for comparison where appropriate.

To sum up, a routine was successfully established to visualize and analyze changes in fluorescence intensities in the microscopy experiments of lipid bilayers on AAO support.

\subsection{Investigation of AMP permeabilization modes using kinetic fluorescence analyses}

To characterize the effects of AMP addition to pore-spanning lipid membranes with the established system, the peptides melittin and magainin-2 were tested.

The influence of the antimicrobial peptides to lipid bilayer permeabilization was tested in dependency on the total peptide concentration in solution. These measurements aimed to shed light on the permeabilization mechanism of the used AMPs, melittin and magainin-2. It was tested if the mechanisms by which the peptides permeabilize the lipid bilayer can be distinguished experimentally from the membrane lysis through Triton-X 100, as presented in 3.3.5.2. It should be shown if the peptides permeabilize the lipid bilayer by forming pores in the membrane. If pore-formation indeed occurred, the idea was to analyze the size of the formed pores by FITC-labeled dextran molecules with different known hydrodynamic radii. 
Only if the formed defect or pore were larger than the hydrodynamic diameter of the fluorophore, the fluorophore would be able to translocate through the membrane into the cavities. From the results with the FITC dextrans it can be estimated how large the formed pores are.

Dye inflow kinetics were tested in a time dependent manner for different peptide concentrations. The total lipid bilayer fluorescence was tracked as an indicator for lipid bilayer lysis. For each measurement, a preparation of a fresh membrane on a different AMP chip was required.

\subsubsection{Kinetics analysis of lipid bilayer permeabilization through magainin-2}

The influence of antimicrobial peptide magainin-2 on lipid bilayer permeability was tested in dependence on peptide concentration and diameter of the aqueous dye. The goal was to obtain information on the size of potentially formed membrane pores and a possible cooperativity of peptides in pore formation.

Pore-spanning lipid bilayers were prepared by spreading POPC GUVs labeled with $0.5 \%$ Texas Red DHPE on porous AAO. After spreading, a water-soluble fluorescent dye was added to the supernatant buffer solution. In the first set of experiments the small dye pyranine was used at $1 \mathrm{mM}$, while magainin-2 concentrations were varied from $0.7 \mu \mathrm{M}$ to $6.6 \mu \mathrm{M}$. In the second set of experiments, FITC labeled dextrans of $40 \mathrm{kDa}$ and $70 \mathrm{kDa}$ were used as bulky fluorescent dyes to investigate the influence of different hydrodynamic radii on the translocation rate. Since a pore-spanning lipid bilayer cannot be used several times, a new preparation of a pore-spanning lipid bilayer on a new AAO chip had to be prepared for each magainin-2 administration. Each measurement for a distinct parameter thus represents an independent experiment. All pore-spanning membranes were prepared under the same conditions.

Using confocal laser scanning microscopy, the aqueous dye fluorescence was imaged in the focal plane underneath a suitable membrane patch. The time-dependent translocation of dye into the AAO cavities was monitored continuously with z-line images taken in time intervals of $30 \mathrm{~s}$. Additionally, the total Texas-Red DHPE fluorescence was monitored to follow lipid bilayer lysis.

After 10 min equilibration, the peptide magainin- 2 was pipetted into the stirred chamber adjusting the total peptide concentration as indicated in the measurements. For each magainin2 experiment, the resulting fluorescence measurements were normalized, standardized and a Boltzmann fit was applied to the resulting curve. Each shown graph represents a characteristic result for a single experiment. To illustrate the filling rate of the pores, the results of the Boltzmann fits are displayed. 
The time-dependent change of relative fluorescence intensity $I_{r e l}(t)$ was measured for several total concentrations ranging from $0.7 \mu \mathrm{M}$ to $6.6 \mu \mathrm{M}$ magainin-2 to determine the concentration dependency of dye translocation kinetics.

\subsubsection{Kinetics of pyranine inflow at different magainin-2 concentrations}
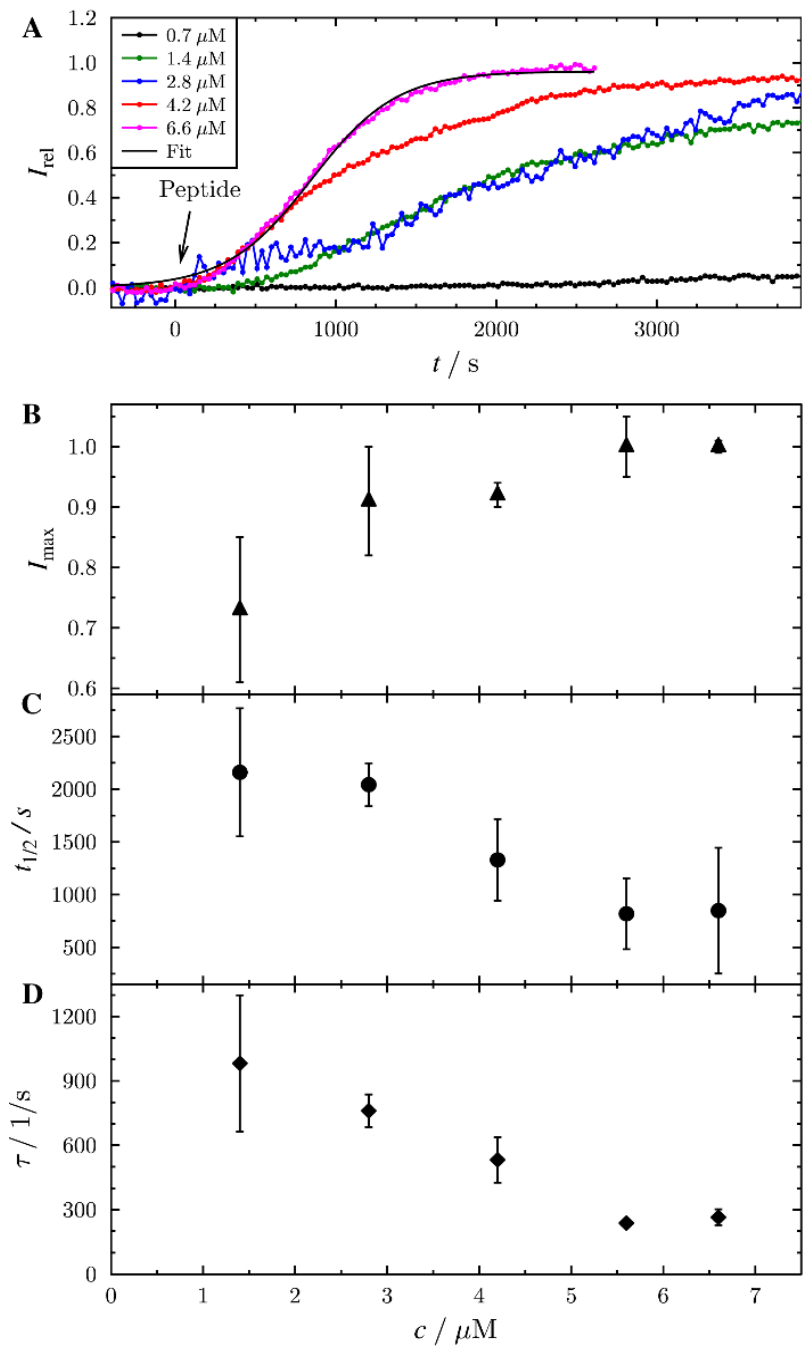

Fig. 3.14: Fluorescence changes of trapped pyranine after magainin-2 addition. (A) Time courses of relative pyranine fluorescence $I_{\text {rel }}$ in membrane-covered substrate cavities at different magainin-2 concentrations in solution. For all experiments, a pyranine concentration of $1 \mathrm{mM}$ was added to the bulk phase. (B) $I_{\max }(\boldsymbol{\Delta})$, (C) center point $t_{1 / 2}(\bullet)$ and (D) $\tau(\bullet)$ as a function of magainin-2 concentration in solution obtained by fitting a Boltzmann-function to the experimental data. For each data point, at least two independent membrane preparations were used. The error bars indicate the standard error of the mean.

The time point of magainin- 2 addition was defined as $\mathrm{t}=0$. A relative fluorescence intensity $\mathrm{I}_{\text {rel }}(\mathrm{t})=1$ represents complete filling of all cavities under the membrane patch. 
At the lowest applied concentration of $0.7 \mu \mathrm{M}$, no increase in fluorescence intensity of pyranine in the cavities was observed. This suggests that, at this concentration, magainin-2 was unable to perforate the membrane.

With increasing peptide concentrations, the degree of cavity filling at the endpoint of the experiment increased, as indicated in Fig. 1.22 (B). Increasing magainin-2 concentrations also led to a faster cavity filling rate, as indicated by decrease of the slope $\tau$ (Fig. 1.22 (D)). The data indicate that magainin-2 has a threshold concentration, which the peptide requires to develop its membrane permeabilization characteristics. Within the tested concentration range it was also observed that there appeared to be a proportional relation between dye translocation speed and magainin-2 concentration.

\subsubsection{Kinetics of membrane fluorescence at different magainin-2 concentrations}

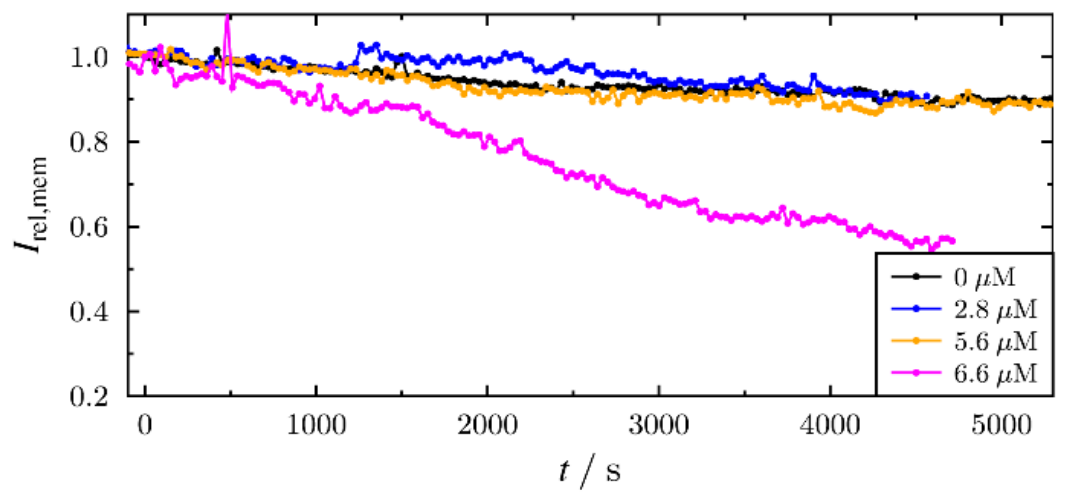

Fig. 3.15 . Time courses of membrane fluorescence intensity of Texas Red DHPE $I_{\text {rel,mem }}$ at different magainin-2 concentrations.

Fig. 3.15 demonstrates that, while at magainin-2 concentrations of $2.8 \mu \mathrm{M}$ and $5.6 \mu \mathrm{M}$ a significant pyranine translocation through the membrane was observed (Fig. 3.14), the membrane fluorescence only decreased at the rate of photo bleaching. This indicates that lipid material is not removed from the surface to a large extent. Only at the highest used magainin2 concentration of $6.6 \mu \mathrm{M}$, a decrease in membrane fluorescence was observed, indicating loss of lipid material from the membrane surface.

Fig. 3.16 shows corresponding $2 \mathrm{D}$ images of a membrane before and after treatment with $6.6 \mu \mathrm{M}$ magainin-2. A slight change in membrane intensity was observed. 


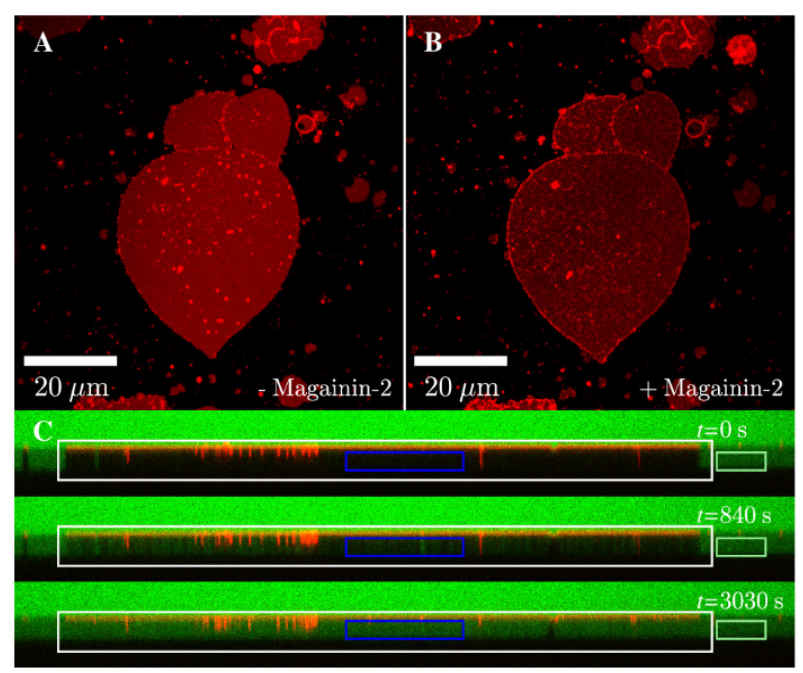

Fig. 3.16 Fluorescence images (top view) of TexasRed DHPE labeled POPC pore-spanning bilayers before (A) and after (B) addition of $6.6 \mu \mathrm{M}$ magainin-2. (C) Overlay of fluorescence images showing $z$ line profiles of pore-spanning TexasRed DHPE labeled POPC bilayers (red) and pyranine (green) at the given time points. Large white box: ROI for membrane fluorescence quantification; small blue box: ROI for pyranine fluorescence quantification; small turquoise box: reference ROI for quantification of pyranine fluorescence. Upon addition of $6.6 \mu \mathrm{M}$ magainin-2 at $t=0 \mathrm{~s}$, pyranine fluorescence (green) becomes visible in the AAO cavities that were sealed by the pore-spanning membrane (red). Scale bar: $20 \mu \mathrm{m}$.

The presented setup allowed to observe the integrity of the membrane itself in a time dependent manner by monitoring the fluorescence intensity $I_{r e l, m e m}$ of the TexasRed DHPE fluorophore. It could be observed that at lower peptide concentrations, the membrane was not fully permeabilized.

The membrane integrity largely remained, even at higher peptide concentrations. Up to a magainin- 2 concentration of $5.6 \mu \mathrm{M}$, no significant loss of lipid material from the membrane was observed, suggesting that magainin-2 does not fully solubilize the membrane.

\subsubsection{Permeability of differently sized dyes through magainin-2 induced membrane disturbance}

To gather further information about the pore sizes that are formed by magainin-2, not only pyranine (Pyr), but also FITC- labeled dextran molecules of $40 \mathrm{kDa}$ (Dex40, Stokes-Einstein radius of $4.5 \mathrm{~nm}$ ) and $70 \mathrm{kDa}$ (Dex70, Stokes-Einstein radius of $6.4 \mathrm{~nm}$ ) were used to monitor their entrance into the AAO cavities upon magainin-2 addition.

In the first experiment, magainin-2 was administered at a concentration of $4.2 \mu \mathrm{M}$, since experiments in 3.4.1.1 had revealed that at this concentration, saturation of cavities with pyranine as dye could be reached.

Fig. 3.17 shows that the translocation of Dex70 was significantly slower than that of pyranine. The half time was shifted from $t_{1 / 2}=1330 \mathrm{~s}$ in presence of pyranine to $t_{1 / 2}=2600 \mathrm{~s}$ in presence of Dex70. Dex70 also showed a significant lag time before considerably 
translocating. Nevertheless, with time the bulky dye was able to pass the lipid membrane through the pores caused by magainin- 2 .

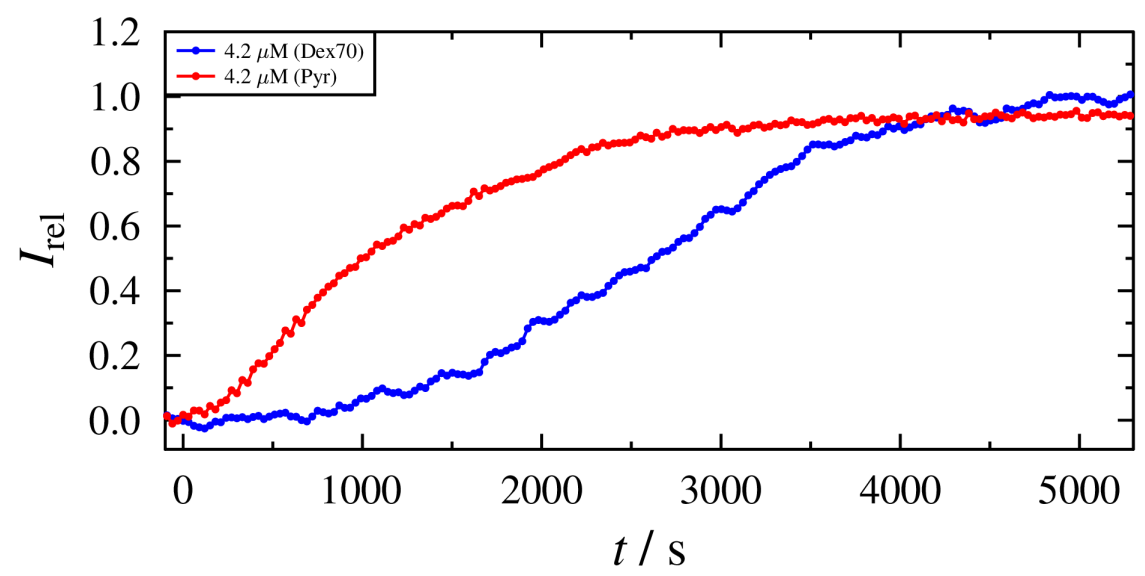

Fig. 3.17: (A) Time courses of $I_{\text {rel }}$ in the presence of pyranine (Pyr) and fluorescein isothiocyanate labeled dextran (FITC-dextran $70 \mathrm{kDa}$, Dex70) after addition of $4.2 \mu \mathrm{M}$ magainin-2.

When a lower magainin-2 concentration of $2.4 \mu \mathrm{M}$ was applied, pyranine could still pass the membrane, while Dex70 was no longer able to do so. (Fig. 3.18). The intermediate-sized Dex40 showed a steady translocation rate across the membrane, but translocation was comparably slow, and occurred at a linear instead of sigmoidal rate.

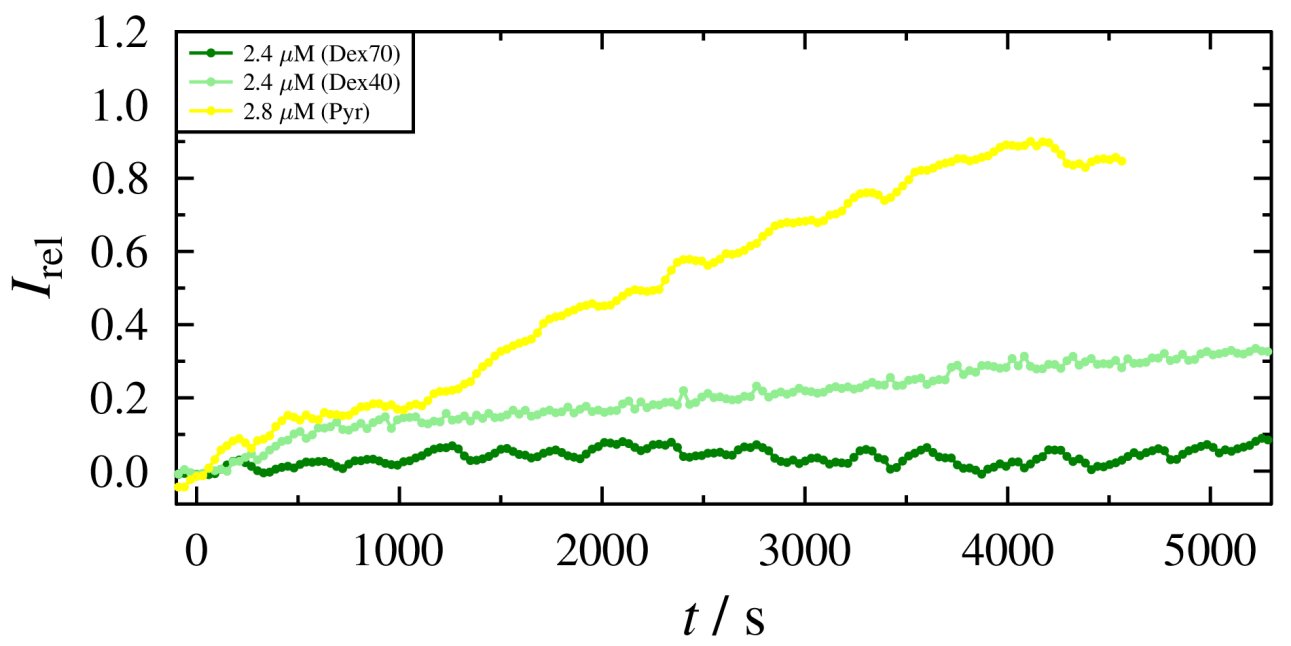

Fig. 3.18: Time courses of Irel in the presence of pyranine (Pyr) and fluorescein isothiocyanate labeled dextran (FITC-dextran $70 \mathrm{kDa}$, Dex70) after addition of $2.4 \mu \mathrm{M}$ or $2.8 \mu \mathrm{M}$ magainin-2, respectively.

As the magainin-2 concentration is lowered even further to $1.4 \mu \mathrm{M}$, none of the dextrans is able to pass the membrane anymore (Fig. 3.19). Meanwhile, pyranine is still able to translocate, indicating that magainin-2 is still able to perforate the membrane. The inability of the bulky dyes to pass is thus probably due to their size. 


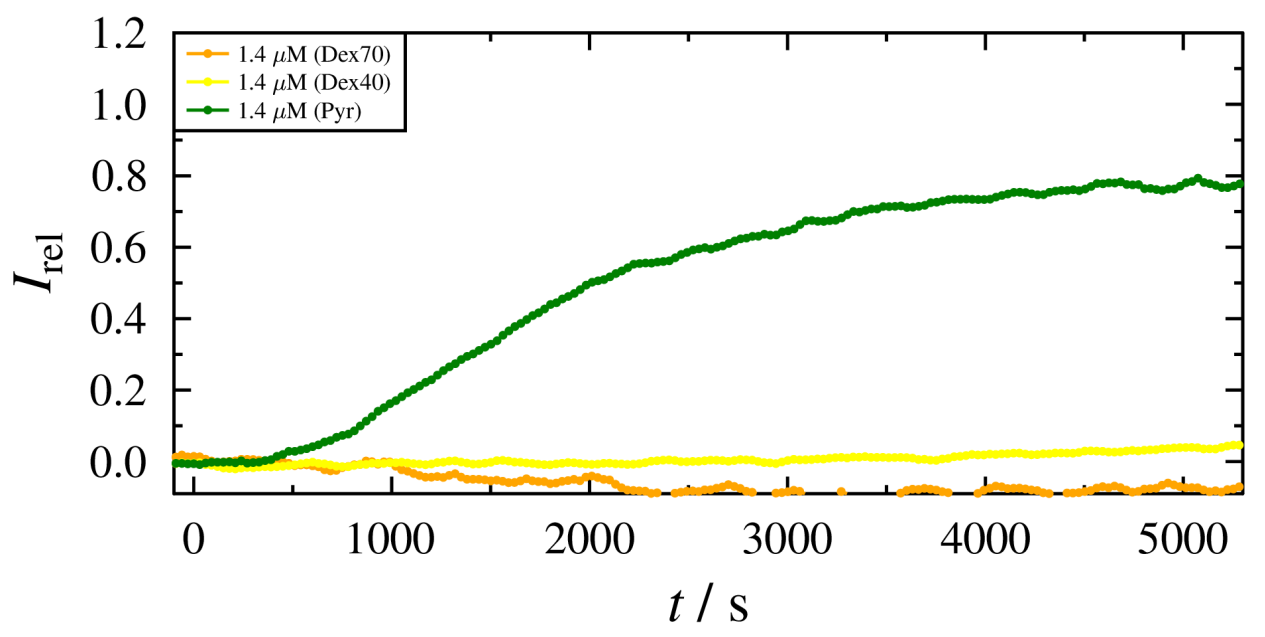

Fig. 3.19: Time courses of $I_{\text {rel }}$ in the presence of pyranine (Pyr) and fluorescein isothiocyanate labeled dextran (FITC-dextran $70 \mathrm{kDa}$, Dex70) at different magainin-2 concentrations.

The translocation behaviors of different dyes at different magainin- 2 concentrations, as discussed in the earlier sections, is summed up in Fig. 3.20. It becomes obvious that only at a relatively high concentation of $4.2 \mu \mathrm{M}$ magainin-2, both pyranine as well as Dex70 are able to pass the lipid membrane to a greater extent. At lower concentrations, dextran translocation stagnates and finally ceases. For better visibility, only the pyranine data of the $4.2 \mu \mathrm{M}$ magainin-2 concentration is shown for reference.

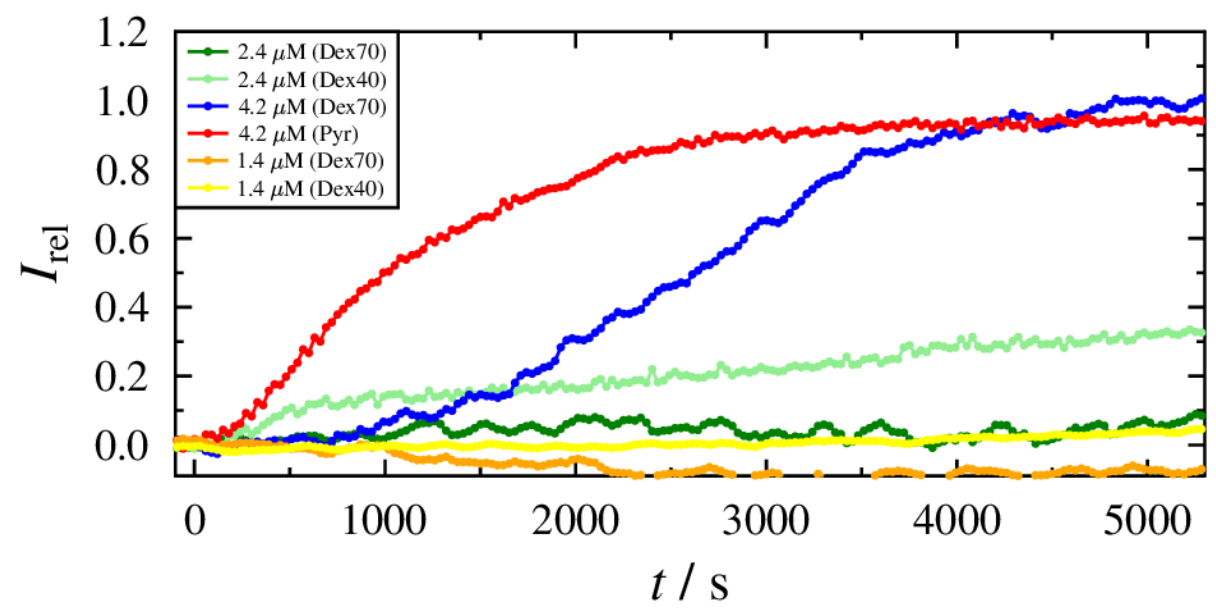

Fig. 3.20: Time courses of Irel in the presence of pyranine (Pyr) and fluorescein isothiocyanate labeled dextran (FITC-dextran 70 kDa, Dex70; and 40 kDa, Dex40) at different magainin-2 concentrations.

To conclude, the careful analysis of membrane behavior upon magainin- 2 administration showed that the peptide requires a certain concentration to develop its membrane-perforating properties, and that peptide concentration is proportional to the size of molecules that may pass a magainin-2 treated membrane. It should also be considered that the inability of 
pyranine to pass a membrane treated with $0.7 \mu \mathrm{M}$ magainin-2, as demonstrated in Fig. 3.14, is also due to size restrictions, and not due to the inability of the peptide to perforate the membrane at this low concentration.

\subsubsection{Kinetics analysis of lipid bilayer permeabilization through melittin}

To investigate whether the developed assay is suited for different AMPs, the sameset of kinetics analyses as in 3.4.1 were performed using melittin.

Pore-spanning lipid bilayers were prepared by spreading POPC GUVs labeled with $0.5 \%$ Texas Red DHPE on porous AAO with $70 \mathrm{~nm}$ pore size. After spreading, a water-soluble fluorescent dye was added to the supernatant buffer solution. In the first set of experiments, the small dye pyranine was used at $1 \mathrm{mM}$, while melittin concentrations were varied from $0.13 \mu \mathrm{M}$ to $1.4 \mu \mathrm{M}$. In a second set of experiments, FITC-labeled dextrans of $40 \mathrm{kDa}$ and $70 \mathrm{kDa}$ at $5 \mu \mathrm{M}$ were used as bulky fluorescent dyes to investigate the influence of different hydrodynamic radii on the translocation rate. This allowed for evaluating the pore size distribution.

Using confocal laser scanning microscopy, the aqueous dye fluorescence was imaged in the focal plane underneath a suitable membrane patch. The time-dependent translocation of dye into the AAO cavities was monitored continuously with z-line images taken in a time intervals of $30 \mathrm{~s}$. Additionally, the total Texas-Red DHPE fluorescence was monitored to follow lipid bilayer lysis.

After 10 min equilibration the peptide melittin was pipetted into the stirred chamber adjusting the total peptide concentration as indicated in the measurements. For each experiment, the resulting fluorescence data was normalized, a Boltzmann fit as applied to the normalized data. Each shown graph represents a characteristic result for a single experiment. To illustrate the filling rate of the pores, the results of the Boltzmann fits are displayed.

The time-dependent change of relative fluorescence intensity $I_{\text {rel }}(t)$ was measured for several total concentration ranges to determine the concentration dependency of dye translocation kinetics. 


\subsubsection{Kinetics of pyranine inflow at different melittin concentrations}
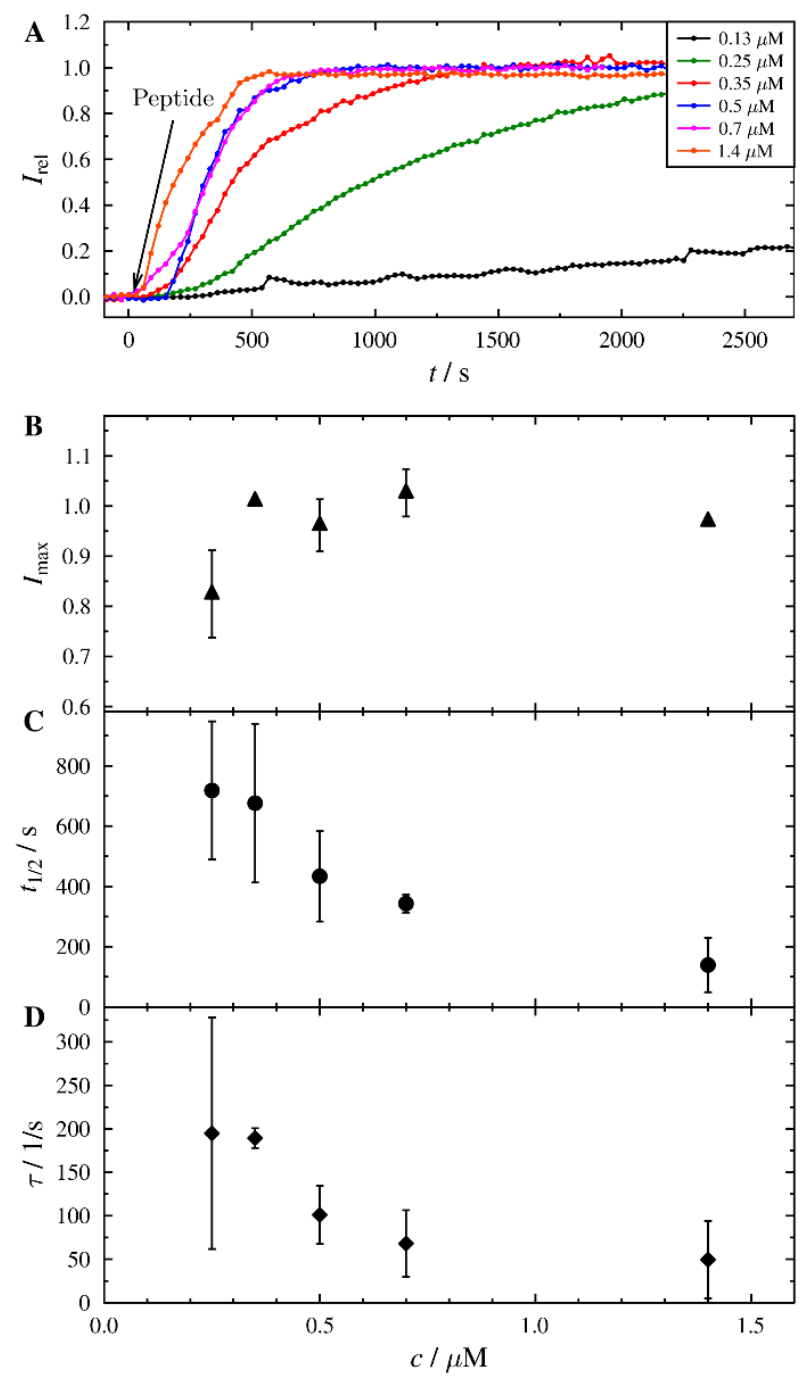

Fig. 3.21: Quantitative measurements and analyses of pyranine fluorescence in cavities below a lipid bilayer patch. (A) Time courses of Irel of pyranine in membrane-covered substrate cavities at different melittin concentrations in solution. For all experiments a pyranine concentration of $1 \mathrm{mM}$ was added to the bulk phase. (B) $I_{\max }(\boldsymbol{\Delta})$, (C) center point $t_{1 / 2}(\bullet)$ and (D) $\tau(\bullet)$ as a function of melittin concentration in solution obtained by fitting a Boltzmann-function to the data. For each data point, at least two independent membrane preparations were used. The error bars show the standard error of the mean.

Fig. 3.21 depicts the obtained analysis results. The time point of melittin addition was defined as $t=0$. A relative fluorescence intensity $I_{\text {rel }}(t)=1$ represents complete filling of all cavities under the membrane patch. At the lowest used concentration of $0.13 \mu \mathrm{M}$ melittin, only very little dye translocation is observed. Increasing the peptide concentration, however, drastically improves the ability of pyranine to pass through the membrane. At $0.5 \mu \mathrm{M}$ maximum pyranine translocation is observed (Fig. $3.21(\mathrm{~A})$ ). The half time $t_{1 / 2}$ as well as the slope $\tau$ at $t_{1 / 2}$ decrease with increasing melittin concentrations (Fig. 3.21 (C-D). In contrast to magainin- 
2 , the decrease appears rather exponential than linear (no quantitative fit). Additionally, $\mathrm{I}_{\max }$ is roughly the same for all concentrations but the lowest (Fig. 3.21 (B)).

\subsubsection{Kinetics of membrane fluorescence at different melittin concentrations}

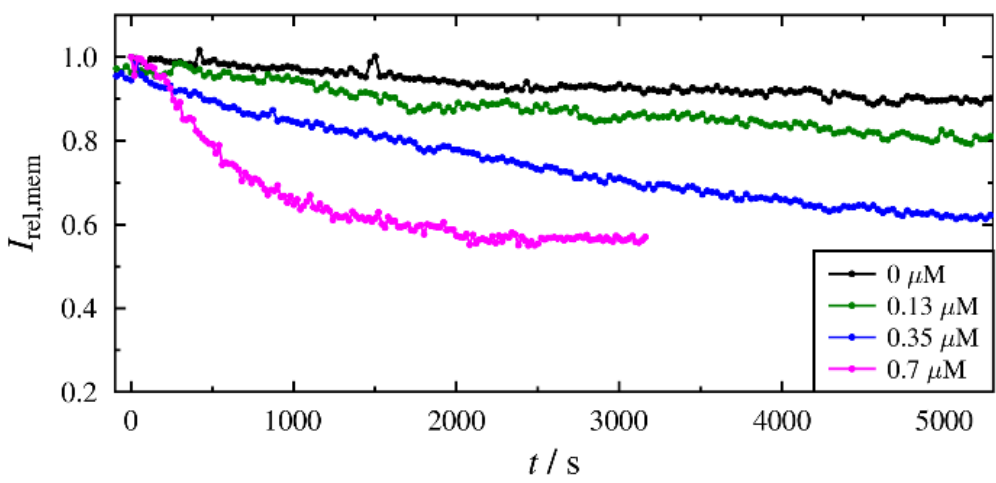

Fig. 3.22: Fluorescence of a pore-spanning membrane after melittin addition over time. Texas Red DHPE fluorescence intensity $I_{\text {rel,mem }}$ at different melittin concentrations.

Fig. 3.22 demonstrates that membrane fluorescence decreases upon melittin treatment. This probably reflects a depletion of lipid material from the membrane. The rate of material depletion is concentration-dependent, and appears linear at melittin concentrations between $0.13 \mu \mathrm{M}$ and $0.35 \mu \mathrm{M}$. At $0.7 \mu \mathrm{M}$ melittin however, fluorescence loss occurs more rapidly, approaching a minimum at about half of the original fluorescence intensity (taking into account photobleaching). Judging from the data, melittin indeed removes lipid from the membrane in a concentration-dependent manner. It does not, however, completely destroy the membrane. Assuming that loss of Texas Red DHPE fluorescence is proportional to loss of lipid material, it appears that melittin removes about half the lipid material and then ceases to exercise its function.

Fig. 3.16 shows corresponding $2 \mathrm{D}$ images of a membrane before and after treatment with a high concentration of melittin $(1.4 \mu \mathrm{M})$. The changes of membrane morphology upon melittin addition were monitored with top view and z-line images. 

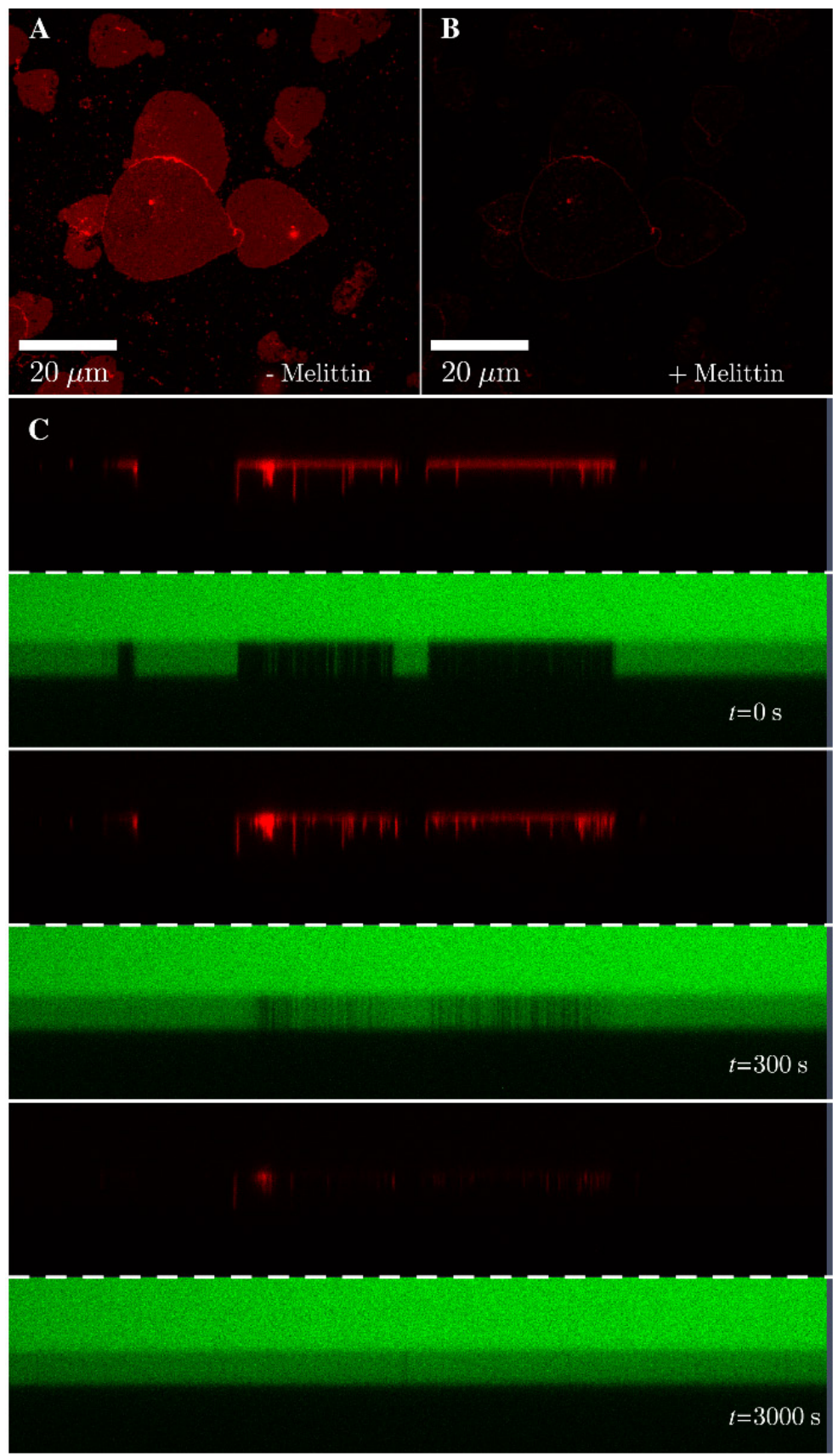

Fig. 3.23: Fluorescence changes of membrane and underlying cavities after melittin addition. Fluorescence images (top view) of TexasRed DHPE labeled POPC pore-spanning bilayers before (A) and after (B) addition of $1.4 \mu \mathrm{M}$ melittin. (C) Fluorescence images showing z-line profiles of pore-spanning TexasRed DHPE labeled POPC bilayers (red, top) and pyranine (green, bottom) at the given time points. Melittin addition leads to fluorescence depletion of the membrane, and dye inflow into the cavities below the membrane patch.

As Fig. 3.23 indicates, the administration of $1.4 \mu \mathrm{M}$ melittin led to significant time-dependent fluorescence reduction on the AAO surface. This suggests a removal of lipid material by the peptide melittin. 


\subsubsection{Kinetics of differently sized dyes after melittin-induced membrane disturbance}

To estimate the formed pore sizes, dye translocation experiments were carried out in the presence of the bulky dye FITC-dextran (70 kDa, Dex70).

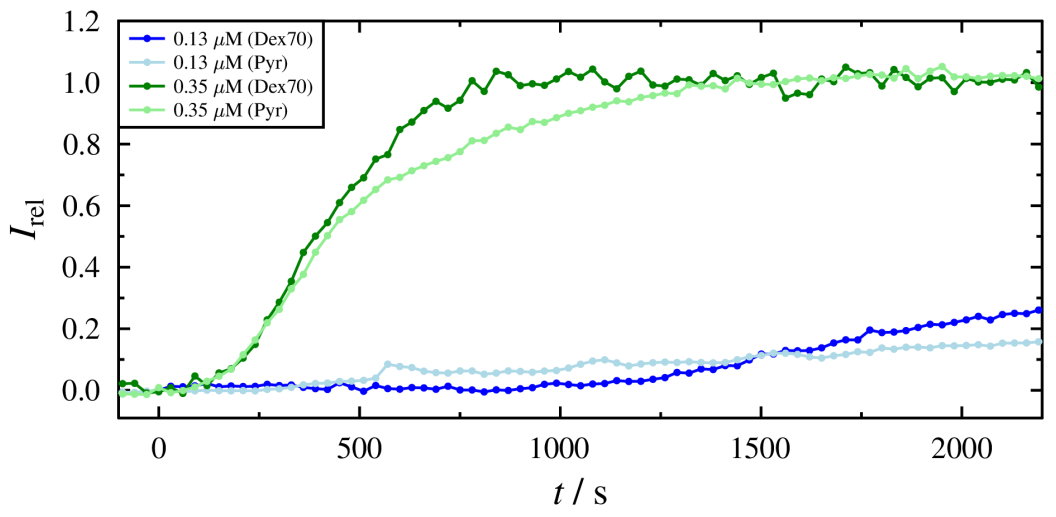

Fig. 3.24: Time courses of $I_{\text {rel }}$ in the presence of pyranine (Pyr, $\left.c=1 \mathrm{mM}\right)$ or FITC-labeled dextran with a molecular mass of $70 \mathrm{kDa}(\operatorname{Dex} 70, c=5 \mu \mathrm{M})$ with different melittin concentrations as indicated.

The different sizes of the two used dyes did not affect their ability to translocate through the melittin-treated membrane (Fig. 3.24). No significant changes in kinetics were observed between Dex70 and pyranine. The dye kinetics are in contrast to the results obtained with magainin-2 (3.4.1). Because both the small and the bulky dye showed the same behavior, the intermediately sized Dex40 was not tested further under these experimental conditions.

\subsubsection{Comparison of melittin and magainin-2 results}

To summarize, the minimum concentration required for a significant permeabilization of the membrane was roughly four times smaller for melittin than for magainin-2. The lag phase between AMP addition and observed membrane permeabilization was much smaller for melittin than for magainin-2.

At a concentration of $1.4 \mu \mathrm{M}$, melittin-induced dye translocation had a 30 fold lower $t_{1 / 2}$ than for magainin-2. In line with this, $\tau$ as measure for the speed by which the dye translocates, was 55-fold larger for melittin as compared to magainin- 2 at the concentration of $1.4 \mu \mathrm{M}$.

This illustrates that melittin causes a membrane permeabilization faster than magainin-2, and other than magainin-2 removes lipid material from the membrane.

The fact that the translocation of the bulky dextran $70 \mathrm{kDa}$ is affected by magainin-2 concentrations, but not melittin concentrations, shows that the two AMPs may use different modes for permeabilizing the membrane. 


\subsection{Phase-separated membranes on AAO support}

Lipid membranes that consist of a certain ternary mixture of lipids can segregate in distinct domains below the phase transition temperature. Phase-separated membranes on solid support have been used to investigate phase-specific lipid interactions of biomolecules before. However, while this approach allows for investigating binding of biomolecules, it lacks a second aqueous compartment on the other side of the membrane. This makes the system unsuitable for addressing questions concerning membrane permeabilization or translocation.

Phase-separated pore-spanning membranes on nanoporous alumina allow for the investigation of the membrane surface and simultaneous monitoring of the aqueous compartments under the respective membrane phases.

Therefore, it was tested whether giant unilamellar vesicles consisting of a ternary phase separated mixture (Sphingomyelin DOPC/cholesterol in a 2:2:1 molar ratio) could be spread on an AAO surface while retaining the phase separation. In case of a successful establishment of the system, the characteristics of the respective phases would be investigated by using antimicrobial peptides, and by monitoring the dye translocation into the underlying compartments.

\subsubsection{Preparation of phase-separated membranes on AAO support}

Giant unilamellar vesicles consisting of sphingomyelin (SM)/DOPC/cholesterol (Chol) in a 2:2:1 molar ratio were prepared and doped with 0.5 mol\% Texas Red DHPE (TR) (40 mol\% $\mathrm{SM} / 39.5 \mathrm{~mol} \% \mathrm{DOPC} / 20 \mathrm{~mol} \% \mathrm{Chol} / 0.5 \mathrm{~mol} \% \mathrm{TR}$ ) on porous AAO substrate with a pore size of $70 \mathrm{~nm}$. This lipid mixture is known to form two lipid phases with different properties, a liquid ordered $\left(l_{o}\right)$ and a liquid disordered $\left(l_{d}\right)$ phase. GUVs were formed in sucrose solution by electroformation at $55^{\circ} \mathrm{C}$, leading to a GUV solution with a total lipid concentration of $0.31 \mathrm{mg} / \mathrm{mL}$. The fluorescence of the membrane was checked with confocal fluorescence microscopy. After successful spreading, the water soluble dye pyranine (green) was added to the supernatant buffer to confirm the impermeability of the formed membrane. While sealed cavities show no fluorescence, the unsealed cavities are filled with pyranine and therefore show fluorescence below the focal plane of the lipid membrane. 


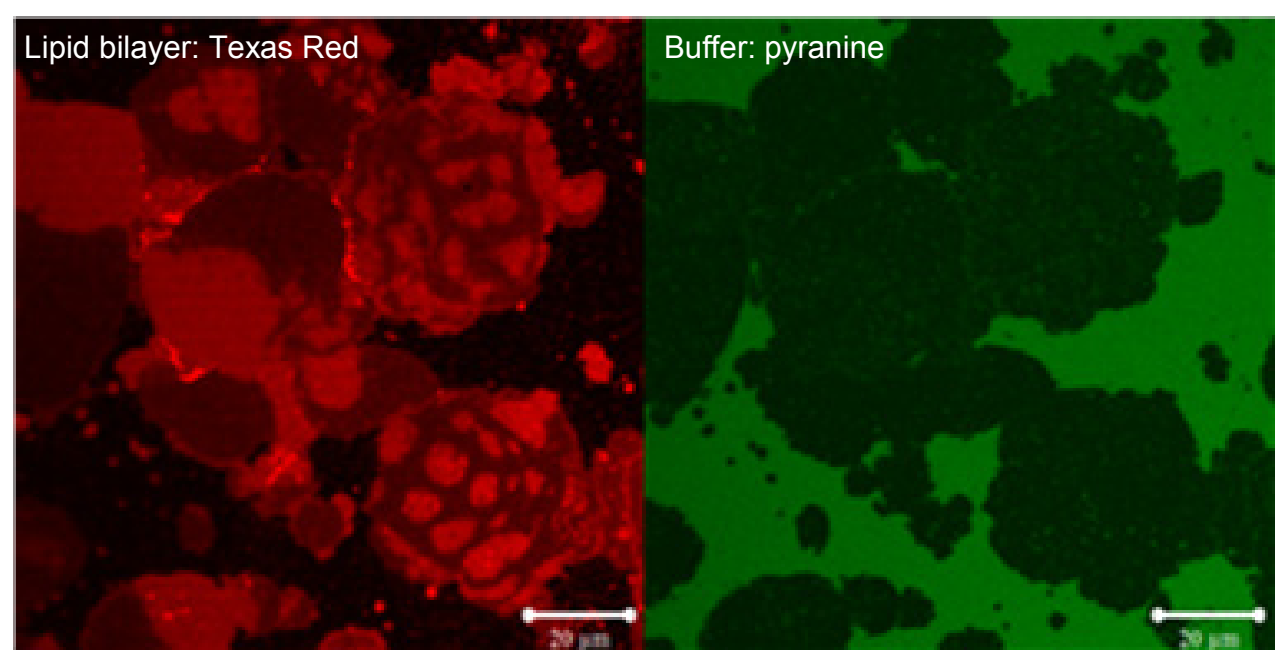

Fig. 3.25: Confocal fluorescence micrograph (top view) of pore-spanning lipid bilayer patches composed of SM/DOPC/Chol/TR $(40 / 39.5 / 20 / 0.5 \mathrm{~mol} \%$, left panel) on AAO support, and fluorescence of aqueous pyranine dye in the corresponding underlying cavities (right panel). The lipid bilayer shows liquid-ordered (lo, dark red) and liquid-disordered domains (ld, bright red). Unsealed cavities appear bright green as they are filled with the pyranine-containing buffer, while the cavities under the lipid bilayer patches remain dark. Membranes seal off the cavities independently from the lipid domains. Light green specks indicate local defects in the sealing of the membrane. Scale bar: $20 \mu \mathrm{m}$.

Spreading of the vesicles led to the formation of lipid bilayer membrane patches as expected (Fig. 3.25). Lipid bilayer membranes showed Texas Red DHPE enriched (light red) and depleted domains (dark red), indicating continued phases separation between $1_{\mathrm{o}}$ and $1_{\mathrm{d}}$ phase. Domains were stable depending on the preparation of the membranes (see following chapters). Residual lipid material could be found on the surface of the AAO. Areas with no lipid material on the surface appear black. The green channel was recorded in the focal plane of the cavities below the lipid membrane patches. Corresponding to the areas without lipid material, these areas showed green fluorescence that is due to filling with pyranine. The cavities under the lipid bilayer patches appeared darker, indicating that these areas were not filled with pyranine. Green specks that appeared in the area covered by lipid membranes indicate filling of single cavities. This might result from defects of the membrane.

To summarize, pore-spanning, phase-separated lipid bilayers could be formed by spreading ternary phase-separated giant unilamellar vesicles onto AAO support. The lipid domains were preserved after spreading. The formed pore-spanning lipid bilayers exhibit Texas Red DHPE enriched and depleted domains. The lipid bilayers are, besides smaller defects, stable and impermeable independently of the lipid domains.

\subsubsection{Spreading of phase separated vesicles in dependency on temperature and lipid amount}

In preliminary experiments it could be shown that spreading of phase separated GUVs while maintaining the phase separation was in principle possible. However, the tests revealed 
varying spreading results. While some lipid membranes showed a sharp segregation of the lipid domains, other preparations led to pore-spanning lipid bilayers with diffuse phases or no phase separation at all.

The phase transition temperature is dependent on the composition of the lipid mixture. To retain separated phases, a temperature below this temperature is necessary. Besides the influence of the temperature, the influence of the used total lipid amount may play a role, and heterogeneity of GUVs may affect the preparations.

Because of this, it was tested which spreading time, lipid amount and temperature while spreading would yield the best conditions for successful GUV spreading with retention of separate phases. Sucrose solution $(300 \mathrm{mM})$ was used and GUVs were formed by electroformation at $55^{\circ} \mathrm{C}$, leading to a GUV solution with a total lipid concentration of 0.31 $\mathrm{mg} / \mathrm{mL}$. Spreading results in dependency on the GUV amount and spreading temperature were observed.

GUVs were added to the chamber and allowed to spread for $15 \mathrm{~min}$. After a buffer exchange to remove residual lipid material from the bulk phase, the topology of the membrane was immediately examined by confocal laser scanning fluorescence microscopy.

Images of the membrane topography were taken in time intervals of $1 \mathrm{~min}$ to observe the membrane stability and phase segregation. In case of stable phase separation, a water soluble fluorescent dye was added to test the impermeability of the membrane.

\subsubsection{Spreading at room temperature with high amounts of lipid material}

Pore-spanning lipid bilayers were prepared by spreading giant unilamellar vesicles (40 mol\% $\mathrm{SM} / 39.5 \mathrm{~mol} \% \mathrm{DOPC} / 20 \mathrm{~mol} \% \mathrm{Chol} / 0.5 \mathrm{~mol} \% \mathrm{TR})$ in PBS buffer $(2.7 \mathrm{mM} \mathrm{KCl}, 136.9 \mathrm{mM}$ $\mathrm{NaCl}, 1.5 \mathrm{mM} \mathrm{KH}_{2} \mathrm{PO}_{4}$, and $\left.8.1 \mathrm{mM} \mathrm{Na}_{2} \mathrm{HPO}_{4}, \mathrm{pH} 7.4\right) .30 \mu \mathrm{L}$ of a giant unilamellar vesicle suspension $(0.31 \mathrm{mg} / \mathrm{ml}$ in $300 \mathrm{mM}$ sucrose $)$ was used for spreading. Vesicles were allowed to spread for $15 \mathrm{~min}$ at room temperature $\left(20^{\circ} \mathrm{C}\right)$. The dynamic morphology changes of the membrane were analyzed by imaging the lipid bilayer patches in time intervals of $1 \mathrm{~min}$. 

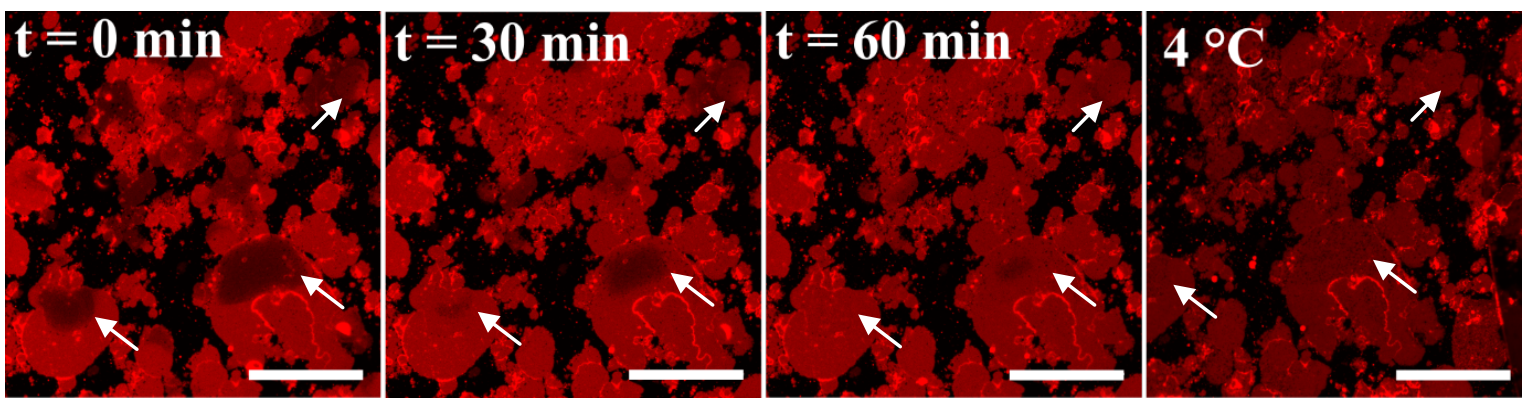

Fig. 3.26: Fluorescence image (top view) of pore-spanning lipid bilayer patches composed of SM/DOPC/Chol/TR (40/39.5/ $20 / 0.5$ mol\%) on AAO support. Membrane patches were prepared at RT from $30 \mu \mathrm{L} 0.31 \mathrm{mg} / \mathrm{ml}$ GUV suspension. $\mathrm{t}=0 \mathrm{~min}$ : Fluorescence immediately after membrane formation. Lipid bilayers segregate into liquid-ordered $\left(l_{0}\right.$, dark red, arrows) and liquid-disordered domains $\left(l_{d}\right.$; bright red, arrowheads). Fluorescence $30 \mathrm{~min}$ after membrane formation indicates desegregation of the domains. Fluorescence 60 min after membrane formation shows almost complete phase mixing. Fluorescence image showing lipid bilayer patches that had been kept at RT after cooling to $4{ }^{\circ} \mathrm{C}$. No re-segregation of the lipid domains could be observed. Membranes show a global loss in fluorescence intensity, indicating membrane damage. Scale bar: $20 \mu \mathrm{m}$.

Fig. 3.26 shows that the amount of vesicles and therefore of deposited lipid material was very high compared to formerly discussed experiments. As a result, the density of adjacent membrane patches was quite high.

The fragmented occurrence of the patches pointed towards excess material in the spreading process, or the spreading of too many GUVs with small size. Modulating the vesicle amount for better spreading results was considered crucial to obtain preparations with better membrane quality. After spreading, a phase separation was observable (Fig. 3.26, arrows). However, the edges of the phases were rather diffuse. After a few minutes, a gradual mixing of the domains could be observed, which continued for about an hour (depending on the initial size of the separated phases). This was despite the fact that the experiment was carried out below the phase transition temperature of the lipid mixture. Subsequent cooling of the substrate to $4{ }^{\circ} \mathrm{C}$ did not lead to recovery of the phase separation but instead to damage to the membrane. This may have been due to stress to the membrane caused by the temperature difference. Because of the observed phase desegregation, no further impermeability tests with pyranine or dextrans were carried out.

\subsubsection{Spreading at $4{ }^{\circ} \mathrm{C}$ with high amounts of lipid material}

It was tested whether the effect of phase mixing as described in 3.5.2.1 would decrease at lower temperatures during spreading. Pore-spanning lipid bilayers were prepared by spreading giant unilamellar vesicles (40 mol\% SM/39.5 mol\% DOPC/20 mol\% Chol $/ 0.5 \mathrm{~mol} \% \mathrm{TR}$ ) in PBS buffer. $30 \mu \mathrm{L}$ of a giant unilamellar vesicle suspension $(0.31 \mathrm{mg} / \mathrm{ml}$ in $300 \mathrm{mM}$ sucrose $)$ was used for spreading. Vesicles were allowed to spread for $15 \mathrm{~min}$ at $4{ }^{\circ} \mathrm{C}$. The dynamic morphology of the membrane was checked by imaging the lipid bilayer patches in defined time intervals of $1 \mathrm{~min}$ at RT. 

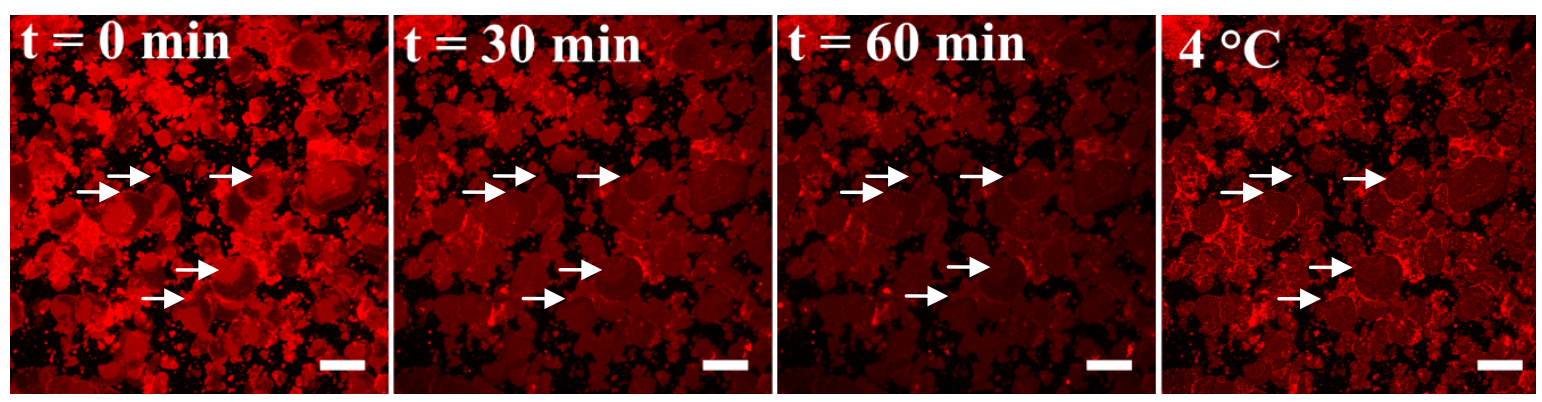

Fig. 3.27: Fluorescence image (top view) of pore-spanning lipid bilayer patches composed of SM/DOPC/Chol/TR (40/39.5/ $20 / 0.5$ mol\%) on AAO support. Membrane patches were prepared at $4{ }^{\circ} \mathrm{C}$ from $30 \mu \mathrm{L} 0.31 \mathrm{mg} / \mathrm{ml}$ GUV suspension. $\mathrm{t}=0 \mathrm{~min}$ : Fluorescence immediately after membrane formation. Lipid bilayers segregate into liquid-ordered ( $1_{0}$, dark red) and liquid-disordered domains ( $1_{\mathrm{d}}$; bright red) Fluorescence $30 \mathrm{~min}$ after membrane formation shows desegregation of the domains. Fluorescence $60 \mathrm{~min}$ after membrane formation shows almost complete phase mixing. No re-segregation of the lipid domains could be observed. Membranes show a global loss in fluorescence intensity, indicating membrane damage. Arrows: phase boarders between $l_{o}$ and $l_{d}$ phase on individual patches. Scale bar: $50 \mu \mathrm{m}$.

The density of patches was still high. Immediately after membrane formation on the AAO support, a phase separation could be observed (Fig. 3.27, arrows). This phase separation was less diffuse compared to the spreading experiment at room temperature (Fig. 3.26). However, phases collapsed in the same manner as described under the previous conditions (3.5.2.1). Due to this membrane behavior, no further tests with pyranine or dextran on membrane impermeability were carried out.

\subsubsection{Spreading at $4^{\circ} \mathrm{C}$ with low amounts of lipid material}

Pore-spanning lipid bilayers were prepared by spreading giant unilamellar vesicles (40 mol\% $\mathrm{SM} / 39.5 \mathrm{~mol} \% \mathrm{DOPC} / 20 \mathrm{~mol} \% \mathrm{Chol} / 0.5 \mathrm{~mol} \% \mathrm{TR}$ ) in PBS. $10 \mu \mathrm{L}$ of a giant unilamellar vesicle suspension $(0.31 \mathrm{mg} / \mathrm{ml}$ in $300 \mathrm{mM}$ sucrose $)$ was used for spreading. Vesicles were allowed to spread for $15 \mathrm{~min}$ at $4{ }^{\circ} \mathrm{C}$. Then, the chamber was rinsed with PBS, and FITClabeled Dex70 $(5 \mu \mathrm{M})$ or pyranine $(1 \mathrm{mM})$ was added to the supernatant to prove the impermeability of the obtained membranes. The results were evaluated by means of fluorescence microscopy by imaging the lipid bilayer patches in time intervals of $1 \mathrm{~min}$ at RT. 

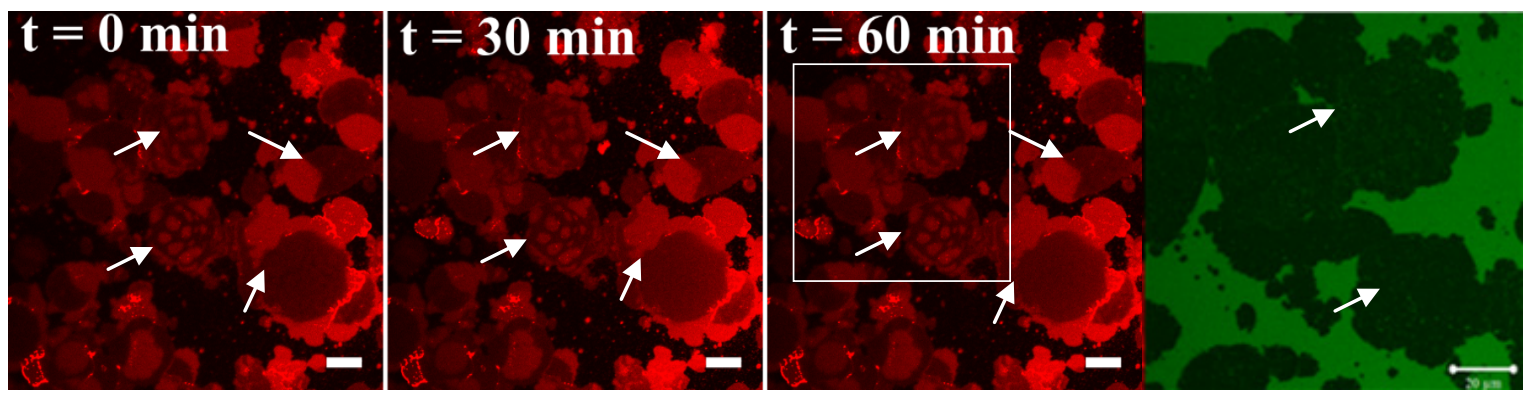

Fig. 3.28: Fluorescence image (top view) of pore-spanning lipid bilayer patches composed of SM/DOPC/Chol/TR (40/39.5/ $20 / 0.5$ mol\%) on AAO support. Membrane patches were prepared at $4{ }^{\circ} \mathrm{C}$ from $10 \mu \mathrm{L} 0.31 \mathrm{mg} / \mathrm{ml}$ GUV suspension. Texas Red fluorescence (panels 1-3, red) and pyranine fluorescence after $60 \mathrm{~min}$ (panel 4, green, enlargement as indicated) are shown. $\mathrm{t}=0 \mathrm{~min}$ : Fluorescence immediately after membrane formation. Lipid bilayers segregate into liquid-ordered $\left(1_{o}\right.$, dark red) and liquiddisordered domains ( $l_{\mathrm{d}}$; bright red). Membrane fluorescence $30 \mathrm{~min}$ after membrane formation indicates stable segregation of the domains. Domains are still segregated after $60 \mathrm{~min}$. Areas below membrane patches show no pyranine fluorescence, indicating the lipid membranes seal the substrate cavities and prevent entry of pyranine. Scale bar: $20 \mu \mathrm{m}$.

As seen in Fig. 3.28, after spreading a sharp phase separation between liquid ordered domains $1_{\mathrm{o}}$ (dark red) and liquid disordered domains $1_{\mathrm{d}}$ (light red) was observed. Even after $60 \mathrm{~min}$ mixing of domains was not observable, showing the long term stability of the separate phases. The reduced lipid amount still leads to high surface coverage with patches, but the number of directly adjacent patches seemed to be reduced.

Pyranine addition confirmed that the membrane patches were impermeable for the small dye. The membrane patches sealed the substrate cavities, independently of the lipid phase.

Only a few membrane defects were seen as visualized by pyranine fluorescence in individual cavities below the membrane patches. Defects appearing as dim patches seemed to occur more often in the lipid disordered phase (qualitative observation). It appeared that locally, the membrane in the liquid disordered phase was somewhat "leaky" (Fig. 3.28 right panel), i.e. allowed a few pyranine molecules to pass without completely losing integrity. Liquid ordered domains, on the other hand, featured defects which had very small diameters and great brightness. These may represent single pyranine-filled cavities, resulting from local membrane defects over individual substrate pores. These types of defects may occur because of close packing of lipids in the lipid ordered phase.

\subsubsection{AMP interaction with specific lipid membrane phases}

\subsubsection{1 $\delta$-lysine effects on phase-separated supported membranes}

After the reaction conditions were determined under which pore-spanning lipid bilayers with stable phase separation are obtained, it was investigated how the antimicrobial peptide $\delta$ lysine interacts with the two different phases. 
For this, pore-spanning lipid bilayers were prepared by spreading giant unilamellar vesicles (40 mol\% SM/39.5 mol\% DOPC/20 mol\% Chol/0.5 mol\% TR) in PBS buffer. $10 \mu \mathrm{L}$ of a giant unilamellar vesicle suspension $(0.31 \mathrm{mg} / \mathrm{ml}$ in $300 \mathrm{mM}$ sucrose $)$ was used for spreading. Vesicles were allowed to spread for $15 \mathrm{~min}$ at $4{ }^{\circ} \mathrm{C}$. Then, the chamber was rinsed with PBS, and FITC-labeled pyranine was added to prove the impermeability of the obtained membranes. The antimicrobial peptide $\delta$-lysine was added to the bulk phase to a final concentration of $70 \mathrm{nM}$. Changes in membrane permeability were followed by imaging the membrane surface and the change in fluorescence intensity of pyranine in the cavities in time intervals of $30 \mathrm{~s}$ at RT.
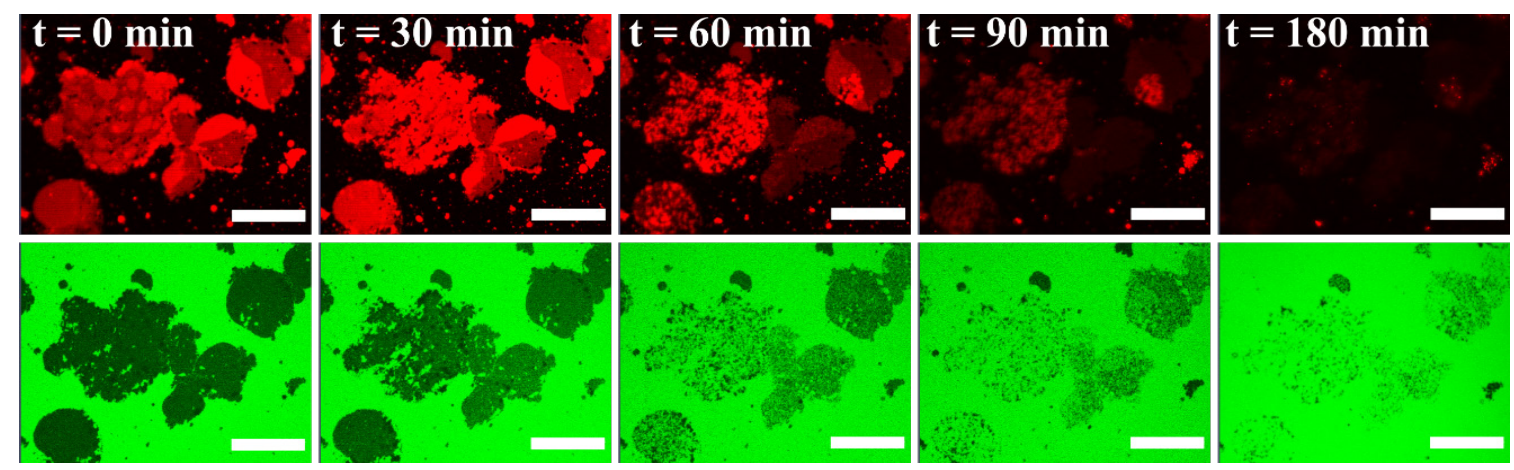

Fig. 3.29: Fluorescence images (top view) of pore-spanning, phase-separated lipid bilayer patches composed of SM/DOPC/Chol/TR (40/39.5/20/0.5 mol\%) on AAO support (red, top row), and corresponding fluorescence images of the aqueous fluorescence dye pyranine in the underlying cavities (green, bottom row). Membrane patches were prepared at $4{ }^{\circ} \mathrm{C}$ from $10 \mu \mathrm{L} 0.31 \mathrm{mg} / \mathrm{ml} \mathrm{GUV}$ suspension. Fluorescence images are shown at different time points after addition $(\mathrm{t}=0)$ of the AMP $\delta$-lysine $(70 \mu \mathrm{M})$. Lipid bilayers are segregated into liquid-ordered $\left(l_{o}\right.$, dark red) and liquid-disordered domains $\left(l_{d}\right.$, bright red). Over time, fluorescence intensity of the lipid bilayers decreases, while pyranine fluorescence in the plane below the membrane increases. Increase is stronger under $l_{d}$ patches than under $l_{o}$ patches. Scale bar: $20 \mu \mathrm{m}$.

The addition of $\delta$-lysine led to a gradual decrease in membrane fluorescence and increase in pyranine fluorescence in the cavities below the lipid bilayer (Fig. 3.29). The AMP appeared to have a preference for the liquid disordered phase, since pyranine fluorescence underneath $1_{d}$ membrane patches increased slightly faster than under $1_{o}$ patches. This observation should be taken with care, as the effect was not always seen clearly in repetitions of this experiment. The decrease in membrane fluorescence suggests a mechanism by which $\delta$-lysine constantly removes lipid material from the lipid bilayer, thereby causing gradual increase in permeability.

\subsubsection{Magainin-2 effects on phase-separated supported membranes}

The influence of the antimicrobial peptide magainin-2 on different domains of a phaseseparated lipid bilayer was investigated.

Pore-spanning lipid bilayers were prepared by spreading giant unilamellar vesicles (40 mol\% $\mathrm{SM} / 39.5 \mathrm{~mol} \% \mathrm{DOPC} / 20 \mathrm{~mol} \% \mathrm{Chol} / 0.5 \mathrm{~mol} \% \mathrm{TR})$ in PBS. $1 \mu \mathrm{L}$ of a giant unilamellar 
vesicle suspension $(0.31 \mathrm{mg} / \mathrm{ml}$ in $300 \mathrm{mM}$ sucrose $)$ was used for spreading. Vesicles were allowed to spread for $15 \mathrm{~min}$ at $4{ }^{\circ} \mathrm{C}$. Then, the chamber was rinsed with PBS, and pyranine $(1 \mathrm{mM})$ was added to prove the impermeability of the obtained membranes. Magainin-2 (3 $\mu \mathrm{M})$ was added and images were taken in a time interval of $1 \mathrm{~min}$ at $\mathrm{RT}$ to observe any Dex70 inflow into the cavities.

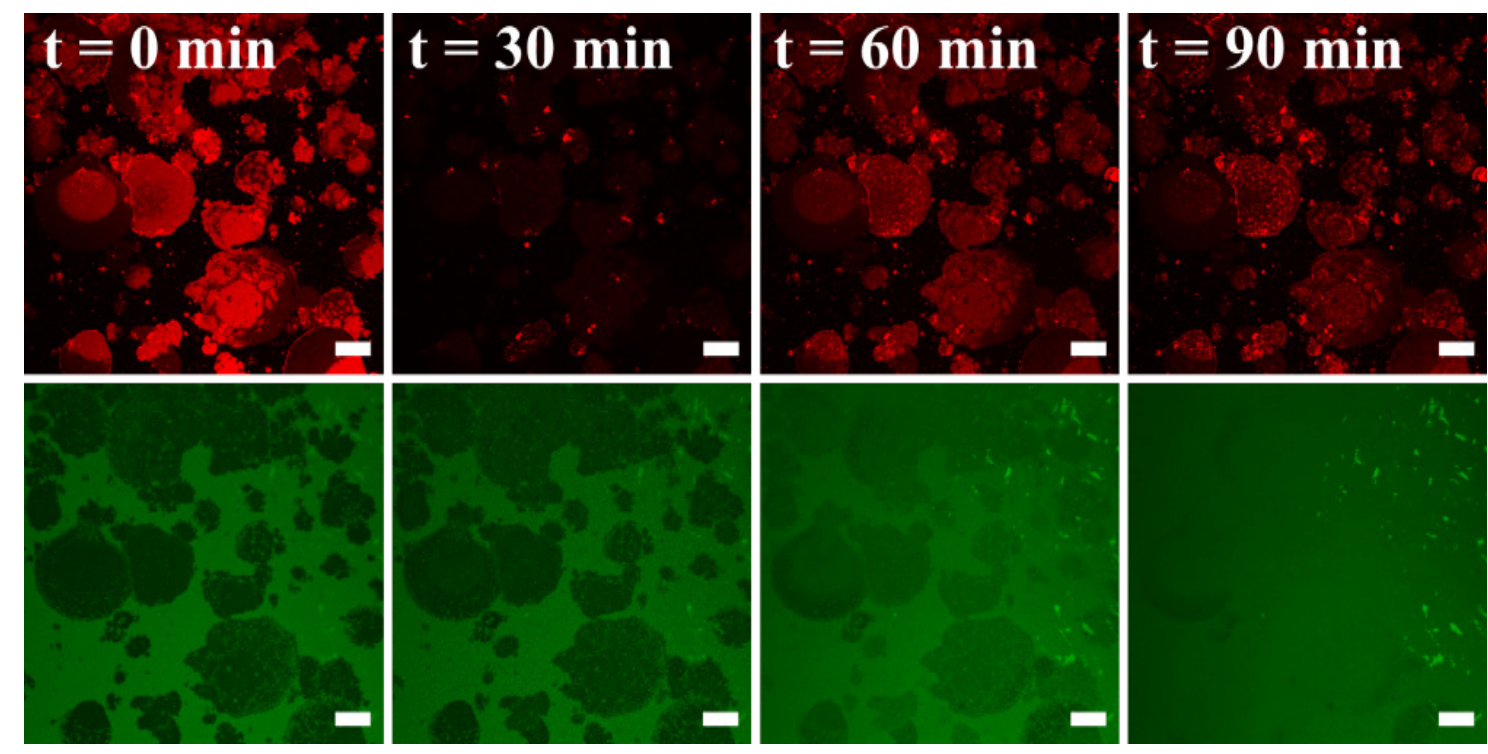

Fig. 3.30: Fluorescence images (top view) of pore-spanning lipid bilayer patches composed of SM/DOPC/Chol/TR (40/39.5/ 20 /0.5 mol\%; red, upper panel) on AAO support, and fluorescence of the FITC-labeled dextran Dex70 (green, lower panel) in the corresponding underlying cavities before and after addition of the AMP magainin-2 $(3 \boldsymbol{\mu M})$. Membrane patches prepared at $4{ }^{\circ} \mathrm{C}$ from $1 \mu \mathrm{L} 0.31$ $\mathrm{mg} / \mathrm{ml} \mathrm{GUV} \mathrm{suspension.} \mathrm{Images} \mathrm{are} \mathrm{shown} \mathrm{at} \mathrm{the} \mathrm{given} \mathrm{time} \mathrm{points} \mathrm{after} \mathrm{magainin-} 2$ addition $(\mathrm{t}=0 \mathrm{~min})$. Upon magainin-2 addition, fluorescence intensity of the phase-separated lipid bilayers decreases, while fluorescence increase in the cavities is observed. The corresponding cavities under the liquid-disordered domains $\left(l_{d}\right.$, bright red membranes) appear to be filled faster. After $90 \mathrm{~min}$, the cavities under the $1_{d}$ domains, are completely filled, while liquid ordered $\left(l_{o}\right)$ domains are filled incompletely. Scale bar: $20 \mu \mathrm{M}$.

The spreading of the GUVs resulted in patches that showed a distinct phase separation that was stable over time (Fig. 3.30). The cavities were sealed by the patches under both liquid ordered $\left(l_{o}\right)$ and liquid disordered $\left(l_{d}\right)$ phase. The cavities under the $1_{o}$ phase showed more defects than under the $1_{d}$ phase. After addition of magainin-2, a gradual filling of the cavities under the membranes could be observed, as indicated by Dex70 fluorescence increase in the focal plane below the membrane patches. Meanwhile, the filling under the $1_{d}$ phase was distinctly faster and ended in complete filling of the cavities after $90 \mathrm{~min}$. Dex70 fluorescence was still lower in the areas below the $1_{\mathrm{o}}$ membrane in comparison to the $1_{\mathrm{d}}$ phase that showed the exact same fluorescence as uncovered substrate areas after $90 \mathrm{~min}$. Overall, magainin-2 appeared to have a much more pronounced effect on the $1_{d}$ phase than $\delta$-lysine. It was aimed to visualize the effect more clearly, and get a fluorescence quantification readout for both phases for a better characterization of the observed effects. 


\subsubsection{Quantitative analysis of selective lipid phase permeabilization induced by magainin-2}

In the previous chapter it was shown that after treatment with magainin-2, a fluorescent dye was capable of entering the cavities under the lipid bilayer. Permeability of the liquid ordered domain appeared hereby increased. This qualitative observation was to be underpinned by quantitative dye inflow measurements. FITC-labeled dextran (70 kDa, Dex70) was used to monitor the membrane permeability, since the rate of dye inflow is dependent on the size and number of the membrane pores that form in the lipid bilayer (3.4.1). The dextran has a larger volume than pyranine, so the change of dye inflow rate was expected to be more pronounced, and easier to distinguish between lipid phases.

Pore-spanning lipid bilayers were prepared by spreading giant unilamellar vesicles (40 mol\% $\mathrm{SM} / 39.5 \mathrm{~mol} \% \mathrm{DOPC} / 20 \mathrm{~mol} \% \mathrm{Chol} / 0.5 \mathrm{~mol} \% \mathrm{TR})$ at $4{ }^{\circ} \mathrm{C}$ in PBS. $1 \mu \mathrm{L}$ of a giant unilamellar vesicle suspension $(0.31 \mathrm{mg} / \mathrm{ml}$ in $300 \mathrm{mM}$ sucrose $)$ was used for spreading. Vesicles were allowed to spread for $15 \mathrm{~min}$ at $4{ }^{\circ} \mathrm{C}$. The chamber was rinsed with PBS, and FITC-labeled Dex70 was added to a final concentration of $5 \mu \mathrm{M}$. Before addition of magainin-2, 2D images were taken at RT in the focal plane of the pore-spanning membrane and in the confocal plane under the lipid bilayer patch. To monitor the rate of dye inflow, zline images were taken in $30 \mathrm{~s}$ intervals at RT before and after addition of magainin-2 $(2 \mu \mathrm{M})$, monitoring the fluorescence of the lipid bilayer membrane and the FITC-dextran. The time point of magainin-2 addition was set to $\mathrm{t}=0$. 
A

- magainin-2

B

+ magainin-2
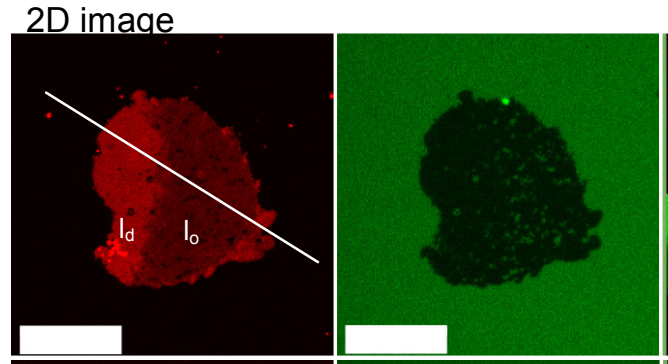

z-line

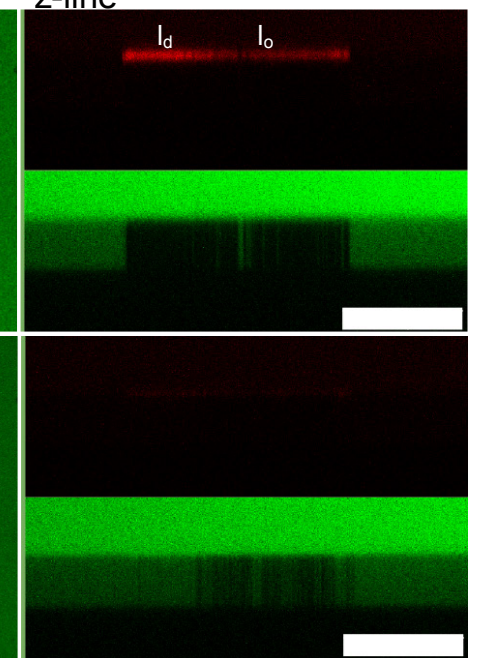

C
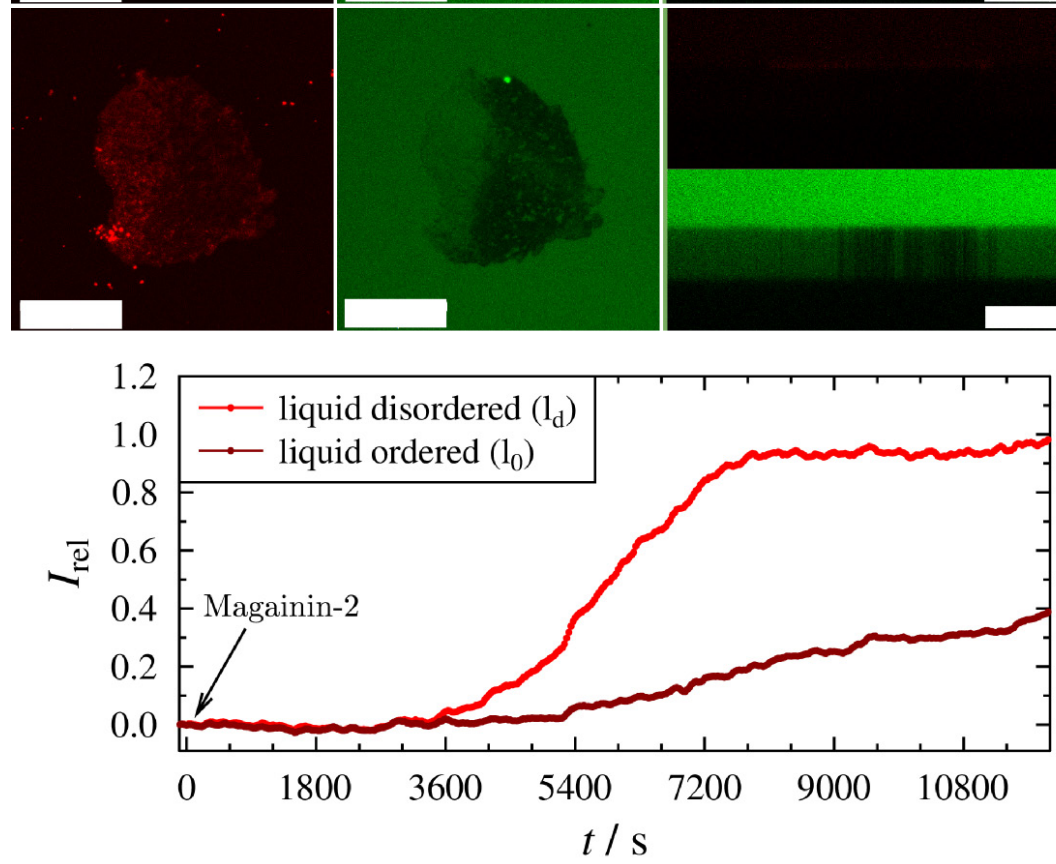

Fig. 3.31: Fluorescence changes of pore-spanning lipid bilayer patches composed of SM/DOPC/Chol/TR (40/ 39.5/ $20 / 0.5$ mol\%) on AAO support upon magainin-2 addition. Membrane patches were prepared at $4{ }^{\circ} \mathrm{C}$ from $1 \mu \mathrm{L} 0.31 \mathrm{mg} / \mathrm{ml}$ GUV suspension. Fluorescence of the pore spanning membranes (Texas Red DHPE, red), and aqueous fluorescence dye FITC dextran $70 \mathrm{kDa}$ (green) in the corresponding underlying cavities before (A) and $3.5 \mathrm{~h}$ after (B) addition of magainin-2 $(2 \mu \mathrm{M})$ with corresponding $z$-line images of the membrane and cavity fluorescence. The $1_{o}$ phase retains some membrane fluorescence, and cavity fluorescence is dimmer compared to the cavities under the $1_{d}$ phase. White line: $z$ line position. Scale bar: $20 \mu \mathrm{m}$. (C) Time course of FITC dextran $70 \mathrm{kDa}$ fluorescence intensity under the $1_{d}$ and $1_{\mathrm{o}}$ phases after addition of $2 \mu \mathrm{M}$ magainin-2. Fluorescence under the $1_{\mathrm{d}}$ phase increases at a higher rate than under the $\mathrm{l}_{\mathrm{o}}$ phase, reaching a maximum about $2 \mathrm{~h}$ after AMP addition.

Despite some defects, both phases of the lipid bilayer patch sealed the underlying cavities (Fig. 3.31 A). The liquid ordered phase $\left(l_{o}\right)$ is dark red, the liquid disordered phase $\left(l_{d}\right)$ is light red, Dex70 fluorescence is presented in green, non-filled cavities appear black. The lo phase appeared to have slightly more local defects than the $1_{d}$ phase, as seen by individual bright spots. The unfilled cavities underneath the membrane showed identical intensity values independently of the overlying lipid phase of the lipid bilayer patch.

The integrity of the lipid bilayer changed after the addition of the peptide, but no mixing of the two phases was observed. Differences between the permeabilization rates of the phases became apparent: After 3.-2 incubation, the cavities clearly showed nearly -complete filling of 
the pores under the $1_{d}$ phase, while dye penetration into cavities below the $1_{o}$ phase remained incomplete (green FITC dextran 70 kDa fluorescence in Fig. $3.31 \mathrm{~B}$ ).

Fig. 3.31 (C) presents the time course of change in Dex70 fluorescence intensity below the respective phase. After a lag phase of about $1 \mathrm{~h}$, the fluorescence intensity of Dex70 st arted to increase for both phases. The fluorescence intensity under the $1_{d}$ phase increased significantly faster than under the $1_{o}$ phase. The fluorescence intensity under the $1_{d}$ phase reached a maximum after about $2 \mathrm{~h}$, while the filling under the phase did not even reach half that intensity by the end of the experiment after $3.5 \mathrm{~h}$. Not only was the dye concentration in the $1_{d}$ phase higher at the end of the experiment, but also the dye inflow rate after magainin- 2 treatment was faster for the $1_{d}$ phase. This shows that magainin-2 has a stronger effect on the lipid disordered membrane. Furthermore, the integrity of the lipid bilayer changed after the addition of magainin-2, but the two phases did not mix.

To summarize the chapter, it is possible in principle to obtain stable phase-separated porespanning membranes by spreading phase-separated GUVs on AAO support.

It was observed that the spreading process of GUVs, which consist of phase-separated lipid domains, has to be controlled more stringently than with homogenous GUVs to retain phase separation. In detail, the amount of lipid material has to be drastically lowered during planar membrane preparation, and low temperatures are required for successful retention of separated phases after spreading. Here, phase transition temperature was $40{ }^{\circ} \mathrm{C}$, and spreading temperature was $4{ }^{\circ} \mathrm{C}$. Otherwise it was observed that the phases were still able to mix, which suggests that the lipid distribution was not at equilibrium at the time of spreading. When the preparation was kept at room temperature, a diffuse separation was observed initially, and phase mixing occurred with time. If spreading was carried out at low temperature but with high lipid amounts, sharp phase edges were obtained but phase mixing still occurred over time. If spread with low amounts of lipid material and at low temperature, the obtained planar membranes were stable and kept sharply separated lipid phases for hours. The experimental conditions under which the spreading occurred thus seemed to have a great influence on the phase stability of the obtained separated phases.

Using phase-separated pore-spanning membranes, it was not only possible to investigate the behavior of the membrane surface of the domains, but also to monitor the aqueous compartments under the respective membrane phases. 
R e s u l t s 


\section{Discussion}

In this work, pore-spanning membranes were evaluated on different supports as a model system for the interaction of antimicrobial peptides (AMPs) with lipid bilayers. Pore-spanning membranes on open pore substrates made of silicon nitride or anodized aluminium oxide (AAO), and closed silica pore substrates were compared.

This work provides groundwork for the development of an on-chip screening platform for AMPs. It also provides means to investigate mechanistic details (permeabilizing properties) of their mode of action by simultaneously monitoring changes in membrane integrity and the transfer of a water soluble dye indicative of the formation of permeabilization pathways through the membrane.

The first chapters will explore general considerations towards all three tested substrate types and permeability assays. These data provide the basis for the discussion of mechanistic evidence of AMP action using the most versatile substrate, and potential applications.

\subsection{Characteristics of lipid bilayer formation on porous substrates}

Sucrose-filled GUVs were exposed to substrates of gold-functionalized closed silicon nitride and open silica pores, as well as silanization-functionalized closed AAO pores. The spreading characteristics of the vesicles were monitored in detail as they formed planar membranes on the porous substrates.

\subsubsection{GUV spreading and planar membrane formation: A detailed view}

This work aimed to characterize the spreading process in detail in order to understand the underlying processes, and to improve yield and quality of the formed substrate-supported membrane bilayers. In previous work [97], pore-spanning membranes had been created with a similar approach and the use of a closed silica pore substrate. However, area coverage yield had been very low.

GUVs spread within minutes to form distinct, heart-shaped membrane patches. Each individual vesicle led to an individual patch rather than forming a homogeneous, combined layer consisting of the lipid material from several vesicles. Brighter lipid areas appeared at the borders of the unilamellar patches, suggesting multilayer deposits that formed during the spreading process [95]. Furthermore, residual lipid material, as well as intact vesicles resting on the surface, were visible as fluorescent dots. 
The observations point out that it is crucial to choose carefully which membrane segments of each preparation are suitable for experimental observations, as the membrane properties may differ even within a patch derived from a single vesicle. Likewise, the properties of two distinct patches may not be completely identical, as they were formed from two individual GUVs and are not connected. Additional fluorescence measurements of the planar membranes could be used in future experiments to ensure comparable membrane properties in sample and control membranes.

With the knowledge about the spreading process, it was possible to qualitatively improve yields of membrane coverage, quality of formed planar membranes, and reproducibility of the sample preparations.

\subsubsection{Closed pores may lead to spherical membrane perturbations}

Closed silica pores exhibited unintended membrane topology changes. During a time span of a few hours after the spreading process, the membranes bulged out over the pores. The perturbation resulted in increased local membrane curvature and the formation of larger reservoirs of unequal sizes underneath the membrane. These were undesired variables that were to be eradicated specifically through the use of a solid supported membrane, as their inconsistent occurrence created unwanted heterogeneity in the preparations. A comparable effect had been noted in previous work [98], but had not been investigated further.

A detailed examination revealed that the peripheral pores of membrane patches showed more bulging of the membrane than the central region. This tendency of the peripheral membrane sections could already be observed in freshly spread patches. The tension on the membranes eventually led to a local detachment of the membrane sections from the substrate, resulting in larger connected cavity areas below the membrane. There appeared to be a force that pushed the membrane out from the substrate surface, leading to membrane protrusions over the pores over time.

A possible explanation for the membrane protrusion over time is the influence of the different osmolarities of the buffers above and below the lipid bilayer. Indeed, the buffer in the cavities had a higher osmolarity, a remnant of the spreading process using dense sucrose buffer. This may have led to the osmotic influx of water and the increase in volume beneath the lipid bilayer, causing the overlying membrane to protrude. During the completion of this work, this hypothesis was comprehensibly addressed by Gleisner et al. [99] who were able to confirm that pore-spanning membranes on cavities can be protruded from the surface by adjusting an osmotic gradient across the membrane, leading to curved membranes. The results were obtained with closed non-functionalized $\mathrm{Si} / \mathrm{SiO}_{2}$ pores with $850 \mathrm{~nm}$ diameter, $4 \mu \mathrm{m}$ rims and $10 \mu \mathrm{m}$ depth, yielding femtoliter-sized cavities underneath each membrane. The group could show that the protruded membrane portion recruits membrane material from the rims of the pores. This way a constant lateral tension is obtained since the membranes are are not 
stretched in $z$ direction [100], and the mechanical behavior of these membranes is similar to planar pore-spanning membranes. To conclude, membrane bulging of pore-spanning membranes on closed pores can be reduced by adjusting the osmolarity of the buffers used for the aqueous compartments above and below the lipid.

The second question concerned why the protrusion effects were more pronounced on the periphery of the membrane patches than in the center. This effect may result from the spreading process of the vesicle on the substrate. It was described that the edges of membrane patches carried multilamellar membrane aggregates. This suggests that the membrane at the edge "rolls in" as it spreads onto the surface. It should also be considered that the transformation of the membrane from spherical to flat implies a distortion of the membrane as it settles onto the substrate. The further the spreading process advances, the more membrane is adhered on the substrate, where its freedom of movement becomes limited. The remaining unattached membrane will experience any perturbation forces due to uneven lipid distribution in inner and outer leaflet, as well as strains that occur as the membrane shape changes from spherical to planar. For this reason, uneven membrane distribution may occur preferentially at the edges of the membrane patches. Another influence on membrane deposition is that the flattening membrane has to displace buffer as it settles onto the support. During the spreading process, the outer areas receive the displaced buffer from the inner areas, which increases the volume of buffer to be displaced on the edges. As a result, more buffer may become trapped inside the cavities at the edge of the membrane patch. The use of larger GUVs may remedy this effect by creating bigger pore-spanning membrane patches with larger areas of homogeneous membrane surface. For that, the preparation of GUVs would have to be optimized to increase the yield of larger GUVs.

One possibility to exploit the effect of peripheral membrane protrusion could be to use it to evaluate in a single sample if membrane reactions occur faster or better on curved membrane (periphery of a membrane patch) or on flat membrane (center). In any case, it should be kept in mind that different zones of the patches may react differently towards membrane perturbing agents.

\subsection{Monitoring material exchange across the membrane: dye release and dye inflow assays}

In order to monitor membrane characteristics, it was an objective to establish an assay where fluorescent dyes could be observed passing through the pore-spanning membrane. It was evaluated if the lipid bilayer on the substrate would keep the reservoirs apart; and if this was the case, an AMP was added to permeabilize the membrane and monitor the dynamics of dye translocation between reservoirs. Since open silicon nitride pores do not feature separated 
buffer reservoirs above and below the membrane, they were unsuitable for this assay. Instead, closed silica and AAO pores were used.

\subsubsection{Closed silica pores facilitate dye release assays}

It was investigated whether a pore-spanning membrane assay would allow monitoring of dye release from the cavities under a lipid bilayer patch upon AMP administration. Closed, goldfunctionalized silica pores with a diameter of $3.5 \mu \mathrm{m}$ were used to trap buffer containing the hydrophilic dye pyranine in the pores, and Texas-Red DHPE-doped GUVs were allowed to spread and seal the pores to form planar pore-spanning membrane patches. It was observed that the membrane seal remained tight after supernatant buffer exchange, an important prerequisite for the assay. However, a considerable rate of pyranine photobleaching was observed.

After melittin addition, pyranine fluorescence in the cavities decreased drastically until values matched those of the supernatant. This strongly suggests that the AMP melittin led to a permeabilization of the lipid bilayer, allowing pyranine to escape from the cavities.

There were certain disadvantages to a dye release assay with closed silica pores. There was only a limited pool of dye molecules available in the individual cavities, which caused severe problems of fluorescence loss since considerable photobleaching was observed. Another problem was the variation of fluorescence intensities of trapped pyranine between preparations and even within preparations: It could not be controlled tightly how much dye was trapped in the pores under the membrane because of local membrane bulging effects. This variance in measurement was exacerbated by the protrusion effects discussed in 4.1.2, where membrane protrusions from the substrate were more prominent and the dye was at a greater distance from the quenching gold surface of the substrate. This resulted in varying fluorescence intensities as an artifact of the membrane bulging, independent from actual changes in membrane characteristics.

Instead of a dye release assay, a dye inflow assay was considered as an alternative. However, this was technically difficult with silica substrate due to drift problems, a different z-position of the substrate surface, and the inability of the microscope to focus deep enough into the pores to monitor dye inflow into the cavities. Most importantly, the fluorescence-quenching gold functionalization of the substrate prevented efficient imaging of the pore interior, which made the gold-functionalized substrate unsuitable for a dye inflow assay.

All in all, the dye release assay as tested in this work was applicable in principle, but not very robust as to reproducibility. For this reason, it was opted for an alternative approach. 


\subsubsection{AAO pores facilitate dye inflow assays}

Based on previous reports [94],[97], AAO substrate was considered as an alternative material for translocation experiments. Because the substrate is optically transparent, it allowed for a dye inflow assay. The dye was added to the supernatant buffer after formation of membrane patches on the AAO substrate. The ability of the dye to enter the cavities indicated which areas of the support were accessible by diffusion. Cavities that were not covered by membranes were filled with dye, whereas membrane-covered cavities remained nonfluorescent. In contrast to the release assay, where a comparably small amount of dye molecules was trapped in the cavities and released upon membrane permeabilization, the large reservoir of dye in the buffer solution over the lipid bilayer patches allowed dye molecules to freely diffuse into the cavities with virtually no depletion from the reservoir. This way, the effect of photo bleaching was minimized.

\subsection{Comparison of the tested porous substrates}

Open silicon nitride pores, closed silica pores, and closed aniodic aluminium oxide (AAO) pores were compared with the focus on experimental versatility.

Pore-spanning membranes on gold-functionalized silicon nitride or silica pores were in principle suitable for the formation of planar lipid bilayers, and allowed for monitoring of the free standing membrane portions. Nevertheless, there were several severe drawbacks that limited the range of readout approaches.

One considerable disadvantage was the fluorescence quenching due to the gold functionalization of the pore surface. Quenching hindered detectability of the membrane on the pore rims. Additionally, the microscope was unable to focus deep enough into the pores. This however was a prerequisite for the establishment of dye inflow experiments to monitor membrane permeabilization.

As an alternative to silica and silicon nitride, substrates consisting of anodic aluminium oxide were applied. The AAO cavity size is significantly smaller with $70 \mathrm{~nm}$ as compared to $1.2 \mu \mathrm{m}$ or $3.5 \mu \mathrm{m}$ for silicon nitride or silica support, respectively.

The advantage of AAO support is that AAO can be hydrophilically functionalized through silanization, and features optical transparency due to the small pore diameter. This characteristic allows for the visualization of the entire lipid membrane and not only the free membrane over the pores, providing a more comprehensive image of any occurring membrane changes. Additionally, this transparency allows for the acquisition of z-stacks. This way, the entire membrane as well as the content of the cavities below the membrane can be visualized three-dimensionally. 
Another prerequisite for dye translocation experiments was a separation of the buffer reservoirs above and below the membrane. This was not the case for open silicon nitride substrate, which made it unsuitable for such an assay. Closed silica pores showed membrane bulging effects that led to very heterogeneous membrane appearance on the substrate. AAO substrate was found to be suitable for dye inflow assays.

Based on these findings, the AAO substrate was identified as the best-suited substrate for the scope of this work, and was used as a substrate in later experiments.

\subsection{Investigation of AMP modes of action on pore-spanning membranes}

The interaction of AMPs with the membranes may lead to several effects: Changes in peptide conformation [45], in-depth membrane localization [101][102], changes in association state [43] or bilayer topology (i.e. pore formation, membrane disintegration; [37][35][103]-[106]. Possible mechanisms that are lethal for the cells are charge neutralization and saturation of bacterial membrane [17].

To test whether the developed pore-spanning membrane approach would be suitable to investigate AMP modes of action on lipid membranes, the two well-known AMPs melittin and magainin-2 were tested on the system.

\subsubsection{Melittin}

\subsubsection{Melittin depletes membrane material on open silicon nitride support}

The addition of the AMP melittin to planar lipid bilayers on open silicon nitride support initially led to disappearance of the multilammellar layers at the edges of the membrane patches, while the general fluorescence intensity of the membrane material decreased. A possible explanation is that the membrane deposits at the rims of patches were more accessible to melittin action because they were structurally less stable due to high membrane curvature and possible exposure of hydrophobic regions. Over time, individual pores abruptly lost their fluorescence. This can be interpreted as pore-by-pore rupture of the membrane covering individual pores. Indeed, SICM micrographs of treated pore-spanning membranes confirmed that the pores in question were no longer covered by membrane [107].

The observations can be interpreted as follows: The lipid material may have been removed by melittin. The remaining lipid material may have at some point been too little to maintain a lipid bilayer, leading to membrane rupture with the remaining material adhering to the inside of the pore. There, it would no longer have been detectable since the membrane fluorescence is quenched by the gold surface. 


\subsubsection{Melittin causes dye release on closed silica pores}

Many AMPs are thought to act by perforating the biomembrane through introduction of local membrane disturbance, leading to an increased permeability of the membrane. These disturbances may be followed by complete membrane rupture or lysis, depending on the peptide. However, almost all peptides are observed to lyse the membrane at a certain peptide concentration [17].

This behavior could be visualized experimentally for melittin using a dye release assay on closed silica pores (3.2.3). Addition of melittin led to a heavy decrease in pyranine fluorescence in the pores, with only a minimal decrease in membrane fluorescence. This suggests that, overall, the membrane stayed intact. Thus, the used assay was able to provide evidence that melittin appears to work not through lysis, but through permeabilization of the membrane.

Higher melittin concentrations $(600 \mathrm{nM})$ led to a pronounced continuous decrease in membrane fluorescence, suggesting material removal from the lipid bilayer. Curiously, it was possible to exploit the previously discussed artifact of membrane bulging (4.1.2) to study membrane changes: It could be observed that melittin addition led to a decrease of the membrane protrusions, as the membrane appeared to "sink in". This observation provided further evidence for an increased material flow across the membrane after melittin addition. By the time the experiment was terminated, all membranes were planar, and some had ruptured.

These observations suggest the succession of several processes: The decrease in membrane fluorescence suggests a removal of membrane material from the lipid bilayer, supporting the findings on open silicon nitride pores (4.4.1.1). The decrease in membrane bulging and the loss of pyranine fluorescence below the membrane indicate that melittin permeabilized the membrane to an extent that allowed the outflow of water or buffer components from the inflated cavities.

The apparent late stage membrane rupturing events upon melittin treatment were observed on closed silica pores similarly to open silicon nitride pores. However, the details and the precise cause of the rupture could not be determined using gold-functionalized substrates because of the quenching of the membrane dye directly on the substrate. Due to this, it could not be determined if lipid material was also removed from the substrate. This issue was addressed on AAO pores.

\subsubsection{Melittin removes lipid from the membrane at a concentration-dependent rate on AAO support}

AAO support provided a substrate with optical transparency that allowed the more detailed investigation of melittin action. Experiments on AAO support confirmed the observations 
made on silicon nitride and silica pores (see previous chapters). Additionally, it was observed that the rate of material depletion from the membrane was dependent on melittin concentration. The membrane fluorescence under influence from melittin decreased in a linear fashion at lower peptide concentrations $(0.13 \mu \mathrm{M}, 0.35 \mu \mathrm{M})$, and exponentially at a higher melittin level $(0.7 \mu \mathrm{M})$. Assuming that loss of Texas Red DHPE fluorescence was proportional to loss of lipid material, it appears that melittin removed about half the lipid material and then ceased to exercise its function. This indicates that melittin removed lipid from the membrane at a concentration-dependent rate without destroying the membrane altogether.

\subsubsection{Melittin concentration influences the dye inflow rate on AAO support}

Interestingly, the size of the used dye did not affect translocation kinetics. Both the small dye pyranine and the bulky dye Dex70 showed comparable membrane translocation kinetics, indicating that the membrane defects by melittin cause enough large pores to allow passing of bulky molecules and small molecules alike.

Similarly to 4.4.1.3, a concentration dependence on dye translocation kinetics was observed. While the lowest used concentration $(0.13 \mu \mathrm{M})$ of melittin led to very little dye translocation, increasing the peptide concentration drastically increased the ability of pyranine to pass through the membrane. At $0.5 \mu \mathrm{M}$ melittin, the maximum pyranine translocation rate was observed. The half time as well as the slope at half time decreased with increasing melittin concentrations. In contrast to magainin-2 (4.4.2.2), the decrease appeared exponential rather than linear. This indicates that the more melittin was used, the faster the dye translocation through a permeabilized membrane was possible. It also showed a cooperativity effect since a certain minimum concentration appeared to be necessary for effective permeabilization of the membrane.

\subsubsection{Summary of melittin effects on pore-spanning membranes}

The data collected from experiments with pore-spanning lipid bilayers on three different substrates (open silicon nitride pores, closed silica pores, closed aniodic aluminium pores) indicate that the antimicrobial peptide melittin is able to permeabilize the membrane and extract material from the lipid bilayer.

The observed threshold concentration for dye translocation suggests a cooperative mechanism of melittin action. It points towards the formation of large pores either as toroidal pores or barrel staves. Additionally, the translocation kinetics were not dependent on the hydrodynamic radii of the aqueous dyes, which implies that the formed pores have a certain minimum size that let all dyes pass. This is in line with reports that melittin acts on lipid bilayers by toroidal pore formation [61]. 
It was also reported that melittin may act like a detergent through the carpet mechanism $[36][40][62][106][108]$. Additionally, it was reported that the secondary structure of melittin seems to be responsible for its hemolytic, but not antimicrobial activity [109]. Melittin diastereomers that lost their helical structure could no longer lyse human erythrocytes but maintained their activity against Gram-positive and Gram-negative bacteria [110]. This has some implications in the context of this work. The membranes used in this work consisted of POPC, a lipid with neutral net charge. In that, they resembled eukaryotic host cell membranes and may also be susceptible to material removal through melittin. This may lead to the point where too little material is left to maintain the integrity of the membrane, so that individual pore-spanning membranes rupture. Possibly, the AMP gets depleted at low melittin concentrations as the melittin molecules stay attached to the extracted lipid material. This way, the dye translocation rate stays linear and this would explain why membrane rupture is only observed at high concentrations of melittin. To summarize, the experiments on different porous substrates using dye translocation assays showed successfully the versatility of the method. They evidence suggests that the activity of melittin may originate from two different modes of action- that of pore formation, and that of material removal.

\subsubsection{Magainin-2}

\subsubsection{Magainin-2 permeabilizes membranes on AAO support but does not remove lipid material}

Magainin-2 was able to facilitate the translocation of aqueous fluorescent dye through the lipid bilayer. In contrast to melittin, the fluorescence of the lipid bilayer stayed constant after magainin-2 administration, and no membrane rupture occurred. This suggests that the mode of action of magainin-2 is solely to form pores or defects in the membrane, not to remove membrane material. These findings contradict previous reports stating that magainin-2 acts according to the carpet model [106].

\subsubsection{Dye translocation rates are proportional to magainin-2 concentration}

It was observed that magainin-2 was not able to cause membrane perforation at very low concentrations of $0.7 \mu \mathrm{M}$. Increased magainin-2 concentrations led to a proportional faster cavity filling rate. These data indicate that magainin-2 has a threshold concentration, which the peptide requires to unfold its membrane permeabilization characteristics. This suggests a cooperativity effect as seen for pore-forming agents.

The increased dye translocation rates could have been caused either by more pores or by larger pores. Here, the use of differently sized dextrans showed that the latter was the case: The translocation of Dex70 was significantly slower than that of pyranine, and Dex70 showed a significant lag time before considerably translocating. This suggests that the pores formed by the assembly of magainin-2 grew with time so that only after a significant lag time, when 
the peptides had arranged into larger pores, the bulky dextran was capable of entering the AAO cavities. If a lower magainin-2 concentration of $2.4 \mu \mathrm{M}$ was applied, pyranine could still pass the membrane while Dex70 was no longer able to do so. This suggests that, at this concentration, magainin-2-induced pores did not grow to a size to allow Dex70 enter the AAO cavities. The intermediate-sized Dex40 showed a steady translocation rate across the membrane, but translocation was very slow, and linear instead of sigmoidal. This was possibly due to the fact that only a limited number of formed pores were large enough to let the intermediate dye pass, slowing its translocation. With that in mind, there is a possibility that the inability of pyranine to pass a membrane treated with $0.7 \mu \mathrm{M}$ magainin-2 may have been due to size restrictions, and not due to the inability of the peptide to perforate the membrane at this low concentration.

All in all these data are in line with the previously published finding that magainins act through toroidal pore formation [56].

\subsubsection{Summary of magainin-2 effects of on pore-spanning membranes}

Magainins are reported to act through toroidal pore formation [56] or the carpet model [106].

The data presented in this work support the model of toroidal pore formation. The size of the alleged pores is proportional to the magainin-2 concentration within the concentration range tested in this work. Indications are that the formed pores may increase in size over time, depending on how much peptide is available for widening of the pores. The presented assay cannot discriminate, however, between the toroidal pore model and the barrel stave model. One approach to address such questions with the presented assay would be to individually label the leaflets of the membranes and observe quenching/dequenching of the dyes if lipids of the inner and outer leaflet mixed during barrel stave formation.

The fact that membrane fluorescence remained relatively constant and that no membrane rupture was observed points against the carpet model. However, magainin-2 did change membrane fluorescence at the highest used concentration of $6.6 \mu \mathrm{M}$. There could be a change in the mode of action at higher concentrations, or the effects could be unspecific due to an excess of magainin-2.

It is interesting to note that a magainin variant was developed under the name pexiganan as antibiotic drug and completed phase 3 clinical trials, albeit not being released [111]. This stresses the relevance of investigating this substance class with a view towards medical applications. The more is known about these AMPs, the better the chances are to develop them into useable drugs. 


\subsubsection{Comparison of melittin and magainin-2 actions on PSM}

Magainin-2 and melittin showed a number of differences in their effects on lipid membranes. The minimum concentration required for a significant permeabilization of the membrane was roughly four times smaller for melittin than for magainin-2. The lag phase between AMP addition and observed membrane permeabilization was also smaller for melittin than for magainin-2.

At a concentration of $1.4 \mu \mathrm{M}$ melittin had a 30 fold lower $t_{1 / 2}$ than magainin-2. In line with this, $\tau$ as measure for the speed by which the dye translocates, was 55 -fold larger for melittin as compared to magainin-2 at the concentration of $1.4 \mu \mathrm{M}$.

This illustrates that melittin causes a membrane permeabilization faster than magainin-2, Other than magainin-2, mellitin removes lipid material from the membrane.

The fact that the translocation of the bulky Dextran $70 \mathrm{kDa}$ is affected by magainin-2 concentrations, but not melittin concentrations, shows that the two AMPs seem to use different modes for permeabilizing the membrane.

\subsubsection{Considerations on the overall versatility of PSM assays to investigate AMP action}

The presented assay illustrated a variety of testing possibilities of a minimal membrane setup to mimic biological membranes. The tested parameters and the possible readouts as well as their functional implications are listed below.

The parameters tested in this work were different porous substrates with different hydrophilic surface functionalizations, AMPs at different concentrations, dye inflow and dye release of differently sized aqueous fluorescent dyes, and changes in lipid bilayer fluorescence over time. It can be assumed that the time from peptide addition to formation of pores is dependent on the insertion efficiency of the AMP into the lipid bilayer, as well as the rearrangement of the peptides within the membrane. With the use of labeled dextrans of different sizes, the pore size can be estimated. Additionally, a time dependency of the pore size can be explored.

The rate of aqueous dye translocation into the substrate cavities is dependent on the size and/or number of formed pores or defects in the lipid bilayer. By determining the rate of dye influx in a concentration-dependent manner, these values can be determined, and compared between substances. It is also possible that the AMPs require cooperativity to unfold their function. In this case, not all cavities would fill at very low AMP concentrations, as not all pore membranes would receive the critical pore-forming AMP concentration. The readout would be that, in equilibrium state, the membrane patch would still show non-filled cavities. For a more general discussion of the advantages and challenges of pore-spanning membrane assays see 4.6 . 
Considering the growing body of knowledge and more differentiated characterization of AMP modes of action [36][40][112], the approach using pore-spanning membranes could be an interesting methodological addition to the means to investigate AMPs further. Together with complementing methods such as computational strategies for the prediction and the design of new active AMPs [113], as well as powerful biosynthesis methods [114], the presented assay could provide the means to screen for active synthetic AMPs.

\subsection{AMPs preferably act on the $l_{d}$ phase of pore-spanning phase- separated membranes}

The established model membrane system on AAO support was used to show that the study of phase-separated pore-spanning membranes on porous support is possible in principle, and to evaluate a possible phase preference, or phase targeting, of AMPs. The preference could be detected through different permeabilization kinetics in different phases.

The geometry and size distribution of $1_{0}$-phase domains in phase-separated membranes on porous support has been reported to depend on temperature, substrate pore size and influence of cholesterol content [115]. This work reports a temperature and concentration dependence during preparation on phase stability. The stably formed domains did not show much mobility over time. Although it was shown that lipid mobility within the membrane is maintained [77][115], it has to be taken into account that the domain patches observed in the presented experiments were spanning numerous pores, so that either the adhesion to the substrate impeded domain movement or the domain size led to the overall appearance of immobile domains even as the domain rims did fluctuate.

After creating, optimizing and characterizing a setup of stable phase-separated pore-spanning lipid bilayers on AAO support, it was possible to test whether AMPs showed a preference for a particular phase in phase-separated membranes. The $l_{d}$ and $l_{o}$ phase were discernible by their difference in fluorescence intensity, which originates from the different density of the membrane material (and with this, density of the fluorophore DHPE-Texas Red). Here, the interactions of $\delta$-lysine and magainin-2 with lipid membrane phases were tested and analyzed quantitatively.

A weak preference of $\delta$-lysine to permeabilize liquid disordered membranes was observed. The effect was not reproducible in all preparations, but very distinct in those where it occurred. Further optimization of the experimental conditions should yield more robust results. It would be interesting to see if the observed defects in the $1_{\mathrm{d}}$ phase after $\delta$-lysine treatment become more numerous over time and if dye fluorescence in the cavities increases or decreases. An according time-lapse experiment could clarify whether the $1_{d}$ phase is in 
itself more permeable, or whether the observed varying permeability is indeed caused by the AMP.

Maganin-2 showed a more pronounced preference of the liquid disordered phase. The fluorescence intensity under the $l_{d}$ phase reached a maximum after about $2 \mathrm{~h}$, while the cavities under the $1_{o}$ phase did not even reach half-maximal fluorescence intensity by the end of the experiment after $3.5 \mathrm{~h}$. The dye inflow rate in the $1_{d}$ phase after magainin- 2 treatment was also higher. These experimental observations indicate that more and/or larger pores were formed by maganin-2 in the liquid disordered phase than in the liquid ordered phase.

These data show that the phase separation can be confirmed not only by the different fluorescent properties of the phases, but also by their differentiated reactions towards AMPs.

A mechanistic explanation for the AMP preference of $l_{d}$ phases may be that the interactions between the lipids in the $1_{d}$ phase are weaker so that it is easier for AMPs to tamper with the lipid-lipid interactions and form pores, micelles, or create other membrane defects. Specifically for magainin-2 it was addressed earlier that the AMP may form pores by oligomerizing within the membrane. The weaker lipid interactions in the $1_{d}$ phase may facilitate easier lateral movement of individual magainin-2 molecules, and larger pores may form easier and faster this way. Interestingly, bacterial membranes are also more fluid, which makes them more susceptible to agents intercalating in the membrane [17].

\subsection{Comparison of pore-spanning membranes with other artificial membrane systems}

\subsubsection{Advantages and challenges of pore-spanning membranes}

The presented system of pore-spanning membranes is very versatile. It allows tailoring of experiments with respect to various parameters: Different substrates make for different pore sizes, lipid compositions can be varied, different buffers above and below the membrane can be utilized and exchanged repeatedly, and microscopic readout in three dimensions over time is possible. With this, the assay provides an excellent minimal system to investigate processes normally occurring on cell membranes.

The assay allows for monitoring changes in the membrane as well as both aqueous compartments. Further experimental variables may be explored to expand the versatility of the presented approach in the future: The lipid composition of the planar membranes may be varied to reflect the composition of physiologically occurring membranes such as prokaryotic or eukaryotic cell membranes or specific organelle membranes. Membrane proteins may be incorporated in the assays, other aqueous dyes with different hydrodynamic properties may be of interest, and quenching/dequenching assays are in principle possible. The assay can be used 
for a variety of applications investigating membrane behavior, for instance budding or fusion events or investigation of agents that alter membrane topology, membrane organization.

Indeed, pore-spanning membrane systems have been used in a variety of applications to investigate biological processes as mentioned: For the investigation of SNARE-mediated single fusion events [82] [83], the $\mathrm{PIP}_{2}$-dependent binding of ezrin and its recruitment of actin filaments [116], binding of collybistin to different phosphoinositides [117]. AAO substrates were used to study molecular recognition events between a receptor and a protein [118], and a porous silicon chip was developed for local electric recordings on model lipid bilayers [76]. Biosensor chips with pore-spanning membranes have been successfully developed for potential high throughput applications [119]-[121] and even made commercially available [122]. It was also possible to spread native cell membranes from epithelial cells on porous support and analyze the membranes through AFM and fluorescence imaging [123][124], pushing the approach towards investigating more physiological conditions. Transport and fusion processes as relevant in cellular membranes were investigated [81]-[83],[125],[126], and it was possible to attach artificial lipid bilayers and cellular membranes [123],[124]. Phase-separated pore-spanning membranes allowed the investigation of binding of a Shiga toxin subunit to its receptor and the lateral organization in the membrane [127]. In the light of these developments, the presented assay provides a valuable addition in generating even more versatility and potential for applications of pore-spanning membrane based high-throughput assays.

The pore-spanning membranes used in this work have several advantages over solid supported phospholipid bilayers. For example, they avoid direct contact with an underlying substrate that can potentially be problematic for the presentation of transmembrane proteins. They also allow solution phase access to both sides of the membrane. Black lipid membranes (BLM) also provide these features, but in the past there were issues of membrane stability and the ddifficulty to manipulate them chemically [74]. Recently, BLM preparation protocols with good reproducibility were reported, and commercially available BLM assays have been used in studies [128]. However, the lipid bilayers of BLMs may still contain residual organic solvents from the preparation process, which may affect the mechanical properties of the membrane [95]. This could also perturb interactions with AMPs.

Compared to vesicle assays, pore-spanning membranes feature membrane planarity and an increased membrane surface size. The membrane is immobilized and can be imaged continuously. Manipulation is possible from both sides.

Some experimental challenges remain when working with pore-spanning membranes.The preparation of the substrate is time-consuming, and GUV spreading does not aways yield satisfactory preparations. Optimization of the spreading process to ensure more homogenous membrane populations with each preparation is therefore a future objective. Due to drift 
problems during time-lapse acquisition, compromises had to be made between maximal acquisition time and xyz resolution.

An experimental limit of the presented assays was also the observation/detection rate. LSM detection imposed time constraints on the measurements since the acquisition of $2 \mathrm{D}$ images could be in the range of minutes. Imaging parameters could be improved in order to achieve a better time resolution and a more detailed dataset of the fluorescence changes during the experiment. Conversely, a more detailed resolution of the occurring processes could be achieved by decreasing the reaction speed by varying temperature, peptide concentrations, or through the use of bulkier dyes.

Some challenges of the closed pore PSM setup are heterogeneities within individual membrane patches. The strong bleaching of the dye in dye release assays on closed pores is unfavorable but can be tolerated as long as reference areas are measured for normalization. Here, the use of a more photostable dye or less harsh imaging conditions could improve the stability of the readout signal.

Finally, it should be kept in mind that the assay presented in this work represents an artificial system which may reflect physiologically observed behavior only to a limited extend. It has to be taken into account that that certain necessary components for the investigated reactions may be missing or be available in excess. For this reason, any scientific evidence obtained with this assay should be validated using complementary methods such as the ones presented in 1.2 .

\subsubsection{Alternative readout methods}

Several possible alternatives readout approaches to study pore-spanning membranes can be used [78], amongst which are atomic force microscopy (AFM) or scanning ion conductance microscopy (SICM). AFM measurements could give insights into the permeabilization mechanism by measuring voltage and penetration depth. The technique was applied successfully to probe elastic properties of pore-spanning membranes such as bending, lateral tension, and stretching [129].

SICM measurements could detect membrane permeabilization by monitoring changes in the ion conductivity [107]. In SICM, a nanopipette carrying an electrode is moved over the specimen in buffer solution, where the counter electrode is placed. The nanopipette detects a current, which becomes restricted as the pipette comes closer to the surface. The surface is scanned by keeping the current constant and by moving the pipette tip accordingly, keeping a constant distance to the specimen. The pipette movement can be translated into topological information on the surface. For the scientific question addressed in this work, a different variant of the SICM principle could be used to detect membrane perforation. The nanopipette could be placed over the membrane at a constant position, and current could be measured. As 
the membrane would perforate, the current would rise because additional ions would be able to pass through from the reservoir underneath the membrane. Even though this experiment was intended, it could not be performed because the necessary control software for the SICM was not available in time. Furthermore, this type of experiment requires a challenging experimental setup where the biomembrane has to be positioned blindly. Additionally, SICM has a low time resolution. To summarize, SICM would be an amperometric alternative for visual interpretation of permeabilization events, but it would not improve time resolution. For instance, it would not be a suitable readout method to monitor the events directly after peptide administration. For this reason, this work focused on improving fluorescence readout as the measuring parameter.

\subsection{Potential applications of pore-spanning membrane assays}

This work aimed to provide basic information about the principle suitability of using porespanning membrane systems to investigate AMPs with respect to mode of action, concentration dependency, and specificity towards certain membrane characteristics. This opens a number of opportunities for practical applications.

\subsubsection{Screening of compound libraries}

The presented approach can be expanded to include membranes of lipid compositions that are characteristic for bacterial pathogens or eukaryotic host membranes. It could be used to screen compound libraries for antimicrobial activity. Further down the development process, derivates of identified compounds could be screened for their efficacy, concentration range, and specificity towards pathogen membranes. Indeed, pore-spanning membrane approaches have already been utilized in studies on targeted drug release [120] and protein detection [119],[125]. Studies on entrapment and triggered release of molecules [120],[130],[131] may prove helpful in the development of automated approaches. Together with complementing methods such as computational strategies for the prediction and the design of new active AMPs [113], as well as effective methods for the biosynthesis of artificial candidate peptides [114], the presented assay could provide the means to study AMP action and identify drug candidates.

\subsubsection{Assessing AMP toxicity towards pathogens versus host cells}

The electrochemical properties of pathogen membranes are crucial for the specificity of AMP action [7]. The large amount of anionic phospholipids in bacterial membranes $[31][32][33][132][133][134][135][136]$ sets them apart from neutral and sterol-enriched mammalian membranes [137][138]. They are also more fluid, which makes them more susceptible to agents intercalating in the membrane. 
Factors that influence AMP activity are peptide concentration in solution, propensity to dimerize/multimerize, membrane composition and fluidity, which is determined by the chemistry and size of the lipid head groups. The transmembrane electric potential and $\mathrm{pH}$ also influence the charged state of peptides and ionic lipids [21]. All these factors can potentially be tested on the presented pore-spanning membrane assay.

By testing prospective AMP candidates on mimicked eukaryotic membranes, it may be possible to pre-determine their hemolytic risks, and modify compounds accordingly to preclude host cell toxicity.

However, it has to be kept in mind that membrane interactions of AMPs on membrane levels in silico or in vitro with model phospholipid bilayers have not been clearly correlated with in vivo data as for instance cell death [139]. This means that it is still not certain that a compound will have therapeutic effects even if it permeabilizes the membrane in the assays such as the one established in this work.

\subsubsection{Overcoming AMP resistances}

Even though only few resistance mechanisms against AMPs are reported, they do exist [62]. Strategies to convey resistance are the production of proteases and peptidases [140][141][142]. Resistance through efflux was also reported [143][144][145][146]. Several AMP resistances due to membrane modifications or though changes in metabolism are reported to date [62],[114]. To counter these resistances, the presented setup of pore-spanning membranes could be used to assess potential AMPs for their efficacy, i.e. in the presence of the according proteases or efflux machinery, or to probe for effective AMPs against modified bacterial membranes.

AMPs act very broadly and can provide a first line of defense against a wide range of pathogens. This property makes them versatile, but often lack potency. The number of therapeutic applications of AMPs to date is still quite low [114],[147] , partially due to a lack of versatile methods to identify and test potential compounds for efficacy and efficiency as well as safety of use. The minimum inhibitory concentration (MIC) for AMPs was reported to be rather high for therapeutic use [148][149], but cooperative effects of AMPs would require a lower overall concentration of the substances [17]. This is why it is makes sense to use AMPs in combination therapy with conventional antibiotics[150], particularly in aiding other agents in overcoming pathogen entry mechanisms such as reduced intake of increased efflux. Such synergistic therapies have been tested with melittin-hybrids and $\beta$-lactamase [151].

AMPs have potential for beneficial use in inflammatory and auto-immune diseases, but very few potential peptides have been evaluated for their therapeutic potential despite the promising indications [21]. One reason is the lack of knowledge concerning potential toxicity 
[152]. Here, the presented assay could provide a means to assays the toxicity in a simply highthroughput approach. 


\section{Appendix}

\subsection{Abbreviations}

AAO Anodic aluminium oxide

AFM Atomic force microscopy

AMP Antimicrobial peptide

BLM Black lipid membrane

Chol Cholesterol

CLSM Confocal laser scanning microscope

Dex10 FITC dextran with $10 \mathrm{kDa}$

Dex40 FITC dextran with $40 \mathrm{kDa}$

Dex70 FITC dextran with $70 \mathrm{kDa}$

DHPE 1,2-Dihexadekanoyl-sn-glycero-3-phospho-ethanolamine

DPPE 1,2-Dipalmitoyl-sn-glycero-3-phospho-ethanolamine

DNA Desoxyribonucleic acid

DOPC 1,2-Dioleoyl-sn-glycero-3-phosphocholine

DOPE 1,2-dioleoyl-sn-glycero-3-phospho-ethanolamine

DTT Dithiothreitol

FITC Fluorescein isothiocyanate

FRAP Fluorescence recovery after photobleaching

GUV Giant unilamellar vesicle

His Histidin

ITO Indium tin oxide

$\mathrm{KCl}$ Potassium chloride

$\mathrm{kDa} \quad$ Kilodalton

LUV Large unilamellar vesicle

MH 6-Mercapto-1-hexanol 
MOPS 3-(N-morpholino)propanesulfonic acid

$\mathrm{o} / \mathrm{n} \quad$ over night

PBS phosphate-buffered saline

POPE 1-Palmitoyl-2-oleoyl-sn-glycero-3-phospho-ethanolamine

POPC 1-palmitoyl-2-oleoyl-sn-glycero-3-phosphocholine

POPE 1-palmitoyl-2-oleoyl-sn-glycero-3-phosphoethanolamine

POPS 1-Palmitoyl-2-oleoyl-sn-glycero-3-phospho-1-serine

PSM Pore-spanning membrane

ROI Region of interest

RT Room temperature

SAM Self-assembled monolayer

SEM Scanning electron microscopy

SICM Scanning ion conductance microscopy

SDS Sodium dodecyl sulfate

SNARE Soluble N-ethylmaleimide-sensitive-factor attachment receptor

SSM Solid supported membrane

SUV Small unilamellar vesicle

TRIS tris(hydroxymethyl)aminomethane

$\mathrm{UV} / \mathrm{vis} \quad$ ultraviolet/visible

\subsection{MATLAB Script for Image Analyses}

The script was created by Dr. Ingo Mey and generously provided for image analyses as described in 2.4.4.

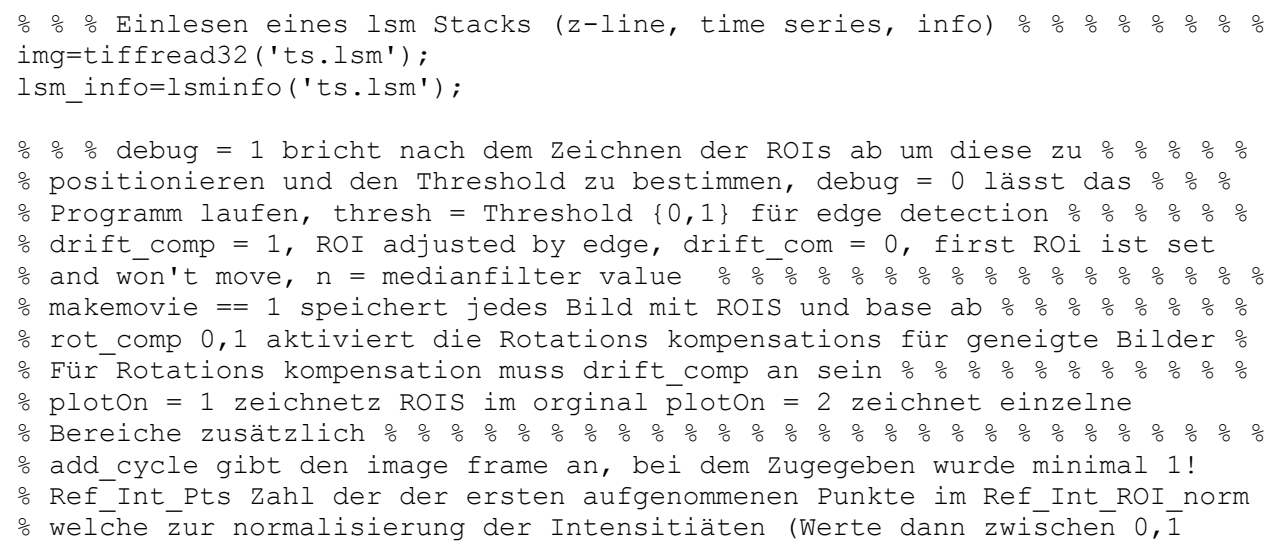


\% genutzt wird. skip cycle kann die ersten \#Value\# Franes überspringen,

\% Referenz ROI Intensität wird dann ebenfalls erst nach den geskippten

$\%$ berechnet und normalisiert Die ausgelassenen Werte haben dann den Wert 0 .

$\circ$ skip cycle $=0$ überspringt nichts $\% \frac{\circ}{\circ} \% \frac{\circ}{\circ} \% \frac{\circ}{0} \% \frac{\circ}{0} \%$

\% baseline $=0$, Ref_Int_Pts werden zur Intensitäts normalisierung benutzt,

o baseline $=1$, jedēr Intensitätspunkt wird auf den entsprechenden

\% Referenzpunkt normalisiert.

\% Baseline 3 normiert korrekt auf totwerte des Sensors!!!!!!!

baseline $=3$;

membrane $=1$

debug $=1$;

ploton $=0$;

if debug $==1$

end

$$
\text { ploton }=1 \text {; }
$$

makemovie $=0$;

SaveData $=1$

drift_comp $=1$;

rot comp $=1$;

thresh $=0.06$;

$\mathrm{n}=2$;

if $\operatorname{rot} \mathrm{comp}==1$;

end

$$
\text { drift_comp }=1 \text {; }
$$

add $\operatorname{cycle}=42$;

skip_cycle $=0$;

Ref_Int_Pts $=10$;

음 Wird NoEdgePoints sehr gros gewählt z.b. 50000, so hat man wieder

\% das tsa2.m, nur werden die ROIs von der anderen Seite gesetzt, daher

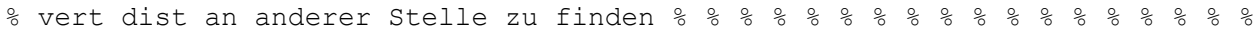

\% NoFit̄pts wird für die Rotation benötigt. Es ist ein $10 t e l$ der Edge Pts

\% Dieser Wert legt fest wieviele Datenpunkte links und rechts für den lin

\% Fit genutzt werden. Nur die äusseren Extreme verhindert eine

\% überbewertung der in der mitte gefundenen Edge Puntke $\frac{\circ}{0} \div \frac{\circ}{0} \div$ NoEdgePts $=400$;

NoFitPts = ceil (NoEdgePts./10);

o Punkte die am Ende ignoriert werden können, um lange Traces zu kürzen ignend=0;

\% 응 Angabe eines Region of Interes in Pixeln, nur Vertikal \% \% $\frac{\circ}{0}$

$\div \%$ ROI werden in absolut werten gesetzt, vert dist angabe in Pixeln

응 für die ROI Höhe, $v$ off ist ein offset Parameter um das ROI von der

응 gefundenen Edge zu Verschieben 응 응 응 응 $\frac{\circ}{\circ} \% \frac{\circ}{0}$

$\mathrm{v}$ off $=-4$

vert dist $\mathrm{ROI}=34$

Ref hor $\mathrm{RO} I=\left[\begin{array}{ll}500 & 540] \text {; }\end{array}\right.$

Img hor $\mathrm{ROI}=\left[\begin{array}{ll}350 & 450\end{array}\right]$;

$\div$ 응 Erstellen leerer Matrizen zum Sammeln der Zeitserienauswertung $\div \frac{\circ}{\circ}$

$\% \div$ Mittels add_cycle wird der Zeitpunkt der Zugabe auf 0 , 0 gesetzt $\% \div$ edge_y_mean_ts=[];

img $\bar{g} r$ int=zeros (size (img, 2)-ignend, size (img, 1));

img_gr_int_norm=zeros ( $\operatorname{size}(i m g, 2)$-ignend, size $(i m g, 1))$;

Ref $\mathrm{ROI}$ in $\bar{t}=$ zeros (size (img, 2)-ignend, size (img, 1));

Ref ROI int norm=zeros (size (img,2)-ignend, size (img, 1));

Img_ROI_int=zeros (size $(i m g, 2)$-ignend, size $(i m g, 1))$;

Img ROI int norm=zeros (size (img, 2)-ignend, size (img, 1));

if membrane $==1$

Img_mem_ROI_int=zeros (size (img, 2)-ignend, size (img, 1));

Img mem ROI int norm=zeros ( $\operatorname{size}(i m g, 2)$-ignend, size $(i m g, 1))$;

Img_mem_Ref_ROI_int=zeros ( $\operatorname{size}($ img, 2) -ignend, size $(i m g, 1))$;

Img mem Ref ROI_int norm=zeros ( $\operatorname{size}(i m g, 2)-i g n e n d, \operatorname{size}(i m g, 1))$;

img gr2 int=zeros ( $\bar{i}$ ze (img, 2)-ignend, size (img, 1));

end

img_gr2_int_norm=zeros (size (img,2)-ignend, size (img, 1));

edge y mean ts=zeros (size (img,2)-ignend, size (img, 1));

time_axis $=(\overline{1}$ sm_info.TimeStamps.Stamps-lsm_info.TimeStamps.Stamps (1));

time axis=time axis-(time axis (add cycle)).

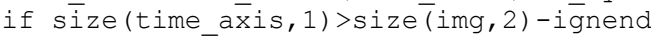
end

time axis $=$ time axis (1:size (img,2)-ignend);

응 MakeMovie options 응 응 응 응 응 응 응 응 응 응 응 응 if makemovie $==1$

cmap $=$ colormap (gray) ; 
maskRefRoi=zeros (size (img (1).data $\{2\}, 1), \operatorname{size}(i m g(1) \cdot \operatorname{data}\{2\}, 2))$; maskImgRoi=zeros (size (img (1).data $\{2\}, 1)$, size (img (1).data $\{2\}, 2))$; Mask=struct;

Images=struct; end

ImagesMem=struct;

응 응 Durchlaufen des Zeitstacks 응 응 응 응 응 응 응 응 응 응 for $i=1+$ skip cycle:size(img, 2)-ignend

$\div \%$ Auswahl des richtigen Kanals in .data $\{x\}$ Übergabe in image_gr $\%$

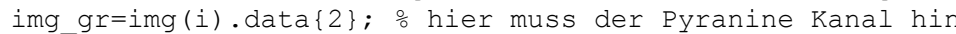

if membrane $==1$ end

img_gr2=img(i).data $\{1\}$; ohier muss der Membran Kanal hin

\% 응 Setzen des Threshhold über edge Funktion $\% \frac{\circ}{0} \% \frac{\circ}{0} \% \frac{\circ}{0}$

\%응 edge (BILD, THRESHOLD), THRESHOLD zwischen 0 und 1 , thresh oben $\frac{\circ}{0}$

if drift comp $==1$

img_bin_edge=edge (medfilt2 (img_gr, [n n ]), thresh) ;

if $\overline{\mathrm{p}} \mathrm{lot} \overline{\mathrm{O}} \mathrm{n}>1$

figure (6)

imshow(img_bin_edge);

end

drawnow

[edge_y, edge_x] =find (img_bin_edge==1);

\% Sortieren des Y-Vektors nach den kleinsten Ziffern,

\% da li oben 1,1 im matlab Bild ist

[edge_y_sort, edge_y_sort_index]=sort (edge_y, 'ascend') ;

if size (edge y sort inde $\bar{x})<$ NoEdgePts

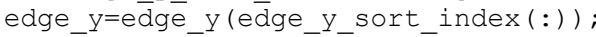

else edge $\mathrm{x}=e^{-} \mathrm{x}$ (edge $\mathrm{y}$ sort ${ }^{-}$index $\left.(:)\right)$;

edge $y=$ edge $y$ (edge y sort index ( 1 :NoEdgePts $))$;

end edge $\mathrm{x}=$ edge $\mathrm{x}($ edge $y$ sort $\mathrm{index}(1$ : NoEdgePts $))$;

if rot comp $==1 \& \&$ isempty (edge y) $==0$

[e'dge_x_rot_sort, edge_x_rot_sort_index] =sort (edge_x, 'ascend')

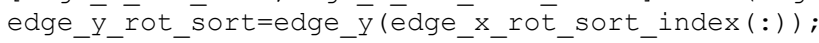

edge $x$ fit $=[$ edge $x$ rot sort $(1:$ NoFitPts $) ; \ldots$

edge $x$ rot sort (end-NoFitPts:end)] ;

edge_y_fit $=[$ edge_y_rot_sort $(1:$ NoFitPts $) ; \ldots$

edge y rot sort (end-NoFitPts:end)] ;

p=polyfit'(edge_x_fit, edge_y_fit, 1);

end

img gr=imrotate $\left(\bar{i} \operatorname{mg} g r,\left(\left(\overline{\operatorname{tatan}}(\mathrm{p}(1)) .{ }^{*} 180\right) . / \mathrm{pi}\right), \mathrm{crop}^{\prime}\right)$;

end

if makemovie $==1$

Images (i).Full=img_gr;

if membrane $==1$

ImagesMem(i). Full=img gr2

end end

edge_y_mean=median (edge_y) ;

edge_y_mean_ts $(i)=e d g e \_$y_mean;

if isnan (edge y mean)

응 $\frac{\circ}{0}$ Hier $\bar{f}$ ange ich leere bilder ab die in z-lines vorkommen $\%$ else

$\%$ \% edge y mean ist double muss zum integer gerundet werden $\%$ edge_mean=zerós (size (img_gr));

edge mean (floor (edge y mean), : ) $=1$;

응 응 Linien erzeugen um den Ref \& Img ROI zu zeichnen 응 응

Ref_x=ones (size (img_gr, 1), 1). *Ref_hor_ROI (1);

Ref $x 2=$ ones (size (img gr, 1), 1) . Ref hor ROI (2);

Ref_y=linspace (1, size (img_gr,1), size (img_gr,1));

Img_x=ones (size (img_gr,1),1).*Img_hor_ROI $(1)$;

Img $x 2=$ ones (size (img gr, 1), 1) . *Img hor ROI (2);

Img_y=linspace (1, sizè (img_gr,1), size (img_gr,1));

응 $\frac{\circ}{0}$ Einzeichnen der begradigten Grenzlinie 응 응 응 응

edge_y_mean_plot=linspace (1,size (img_gr,2), size (img_gr,2));

edge_x_mean_plot=edge_y_mean_plot;

edge y mean plot=edge y mean;

edgy_y_mean_round=floor(edge_y_mean) -v_off ;

응 Anzeige des orginals mit Grenzlinie aus edge Fkt 응 $\frac{0}{0}$

if ploton >0

응 figure (6)

\% imshow(img_gr)

figure (1) 


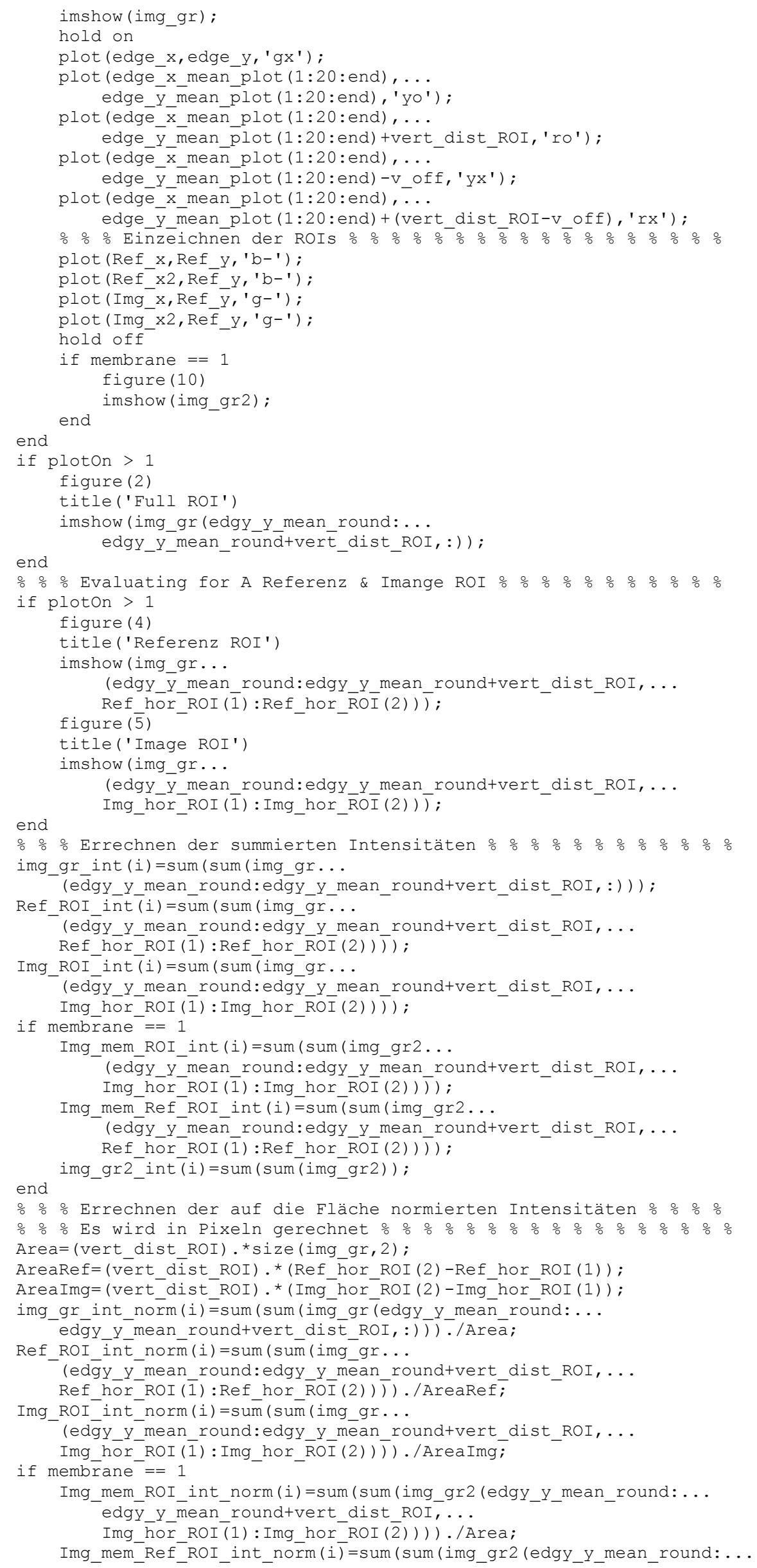




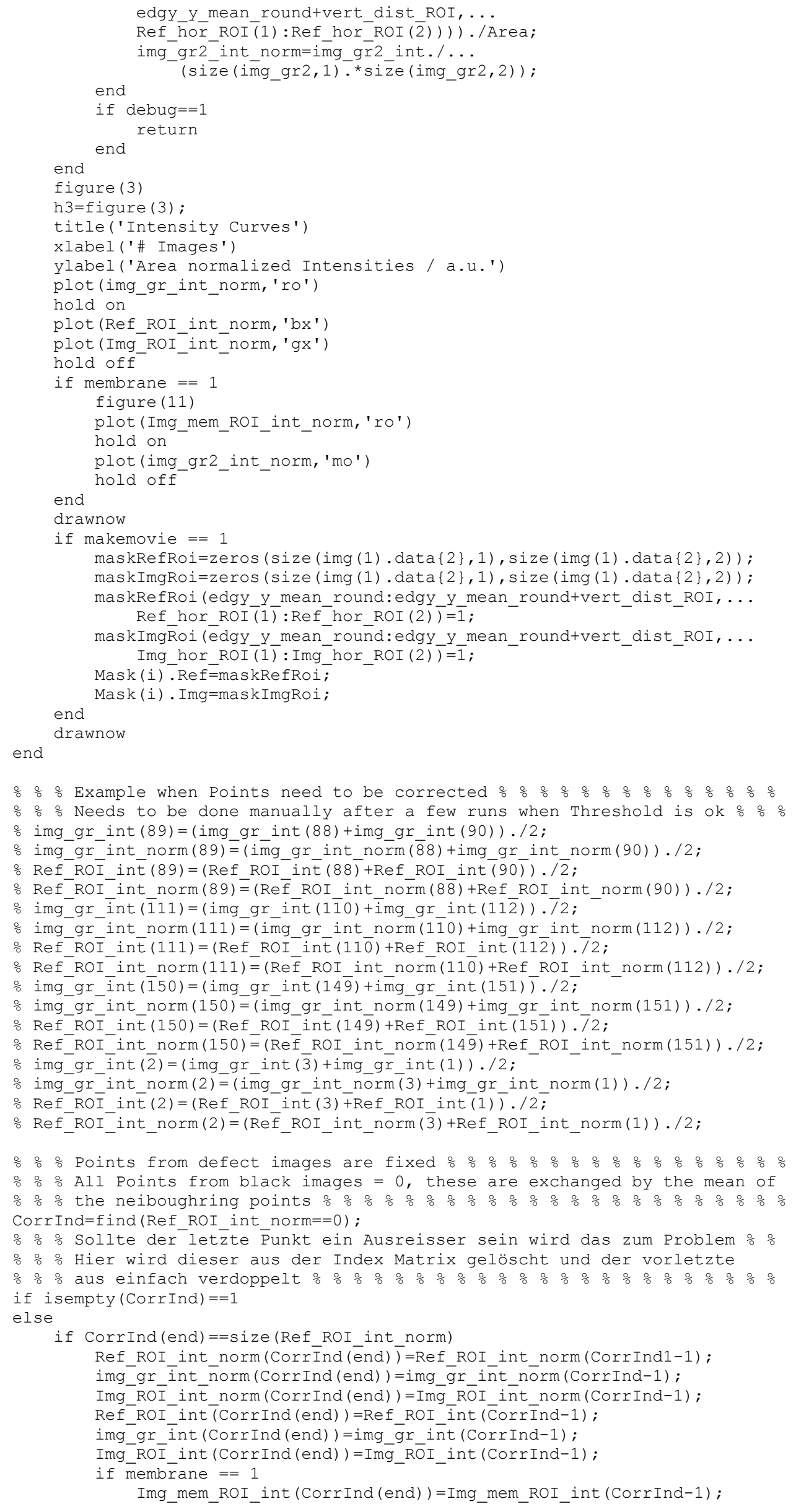




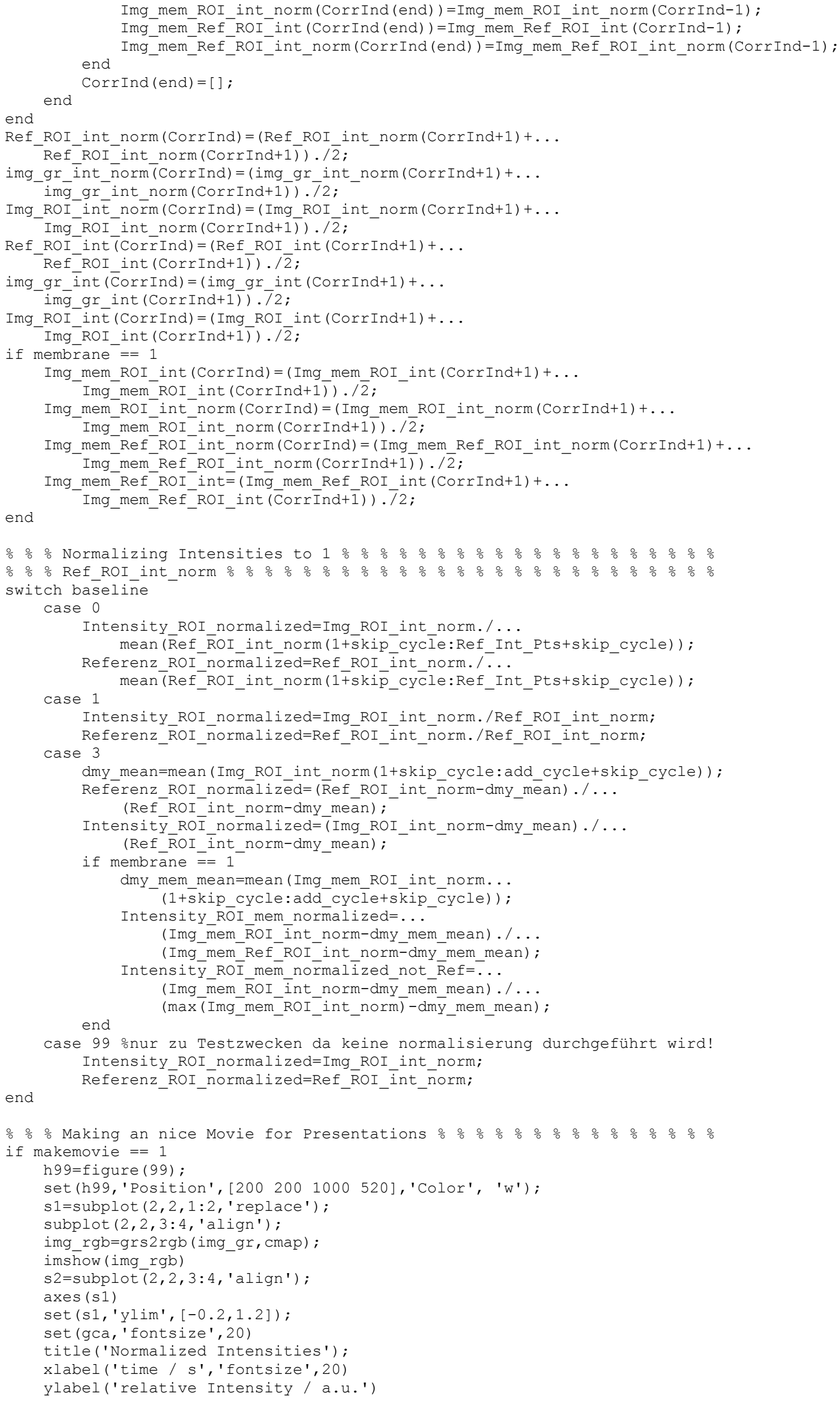

case 99 onur zu Testzwecken da keine normalisierung durchgeführt wird! Intensity ROI normalized=Img ROI int norm; 


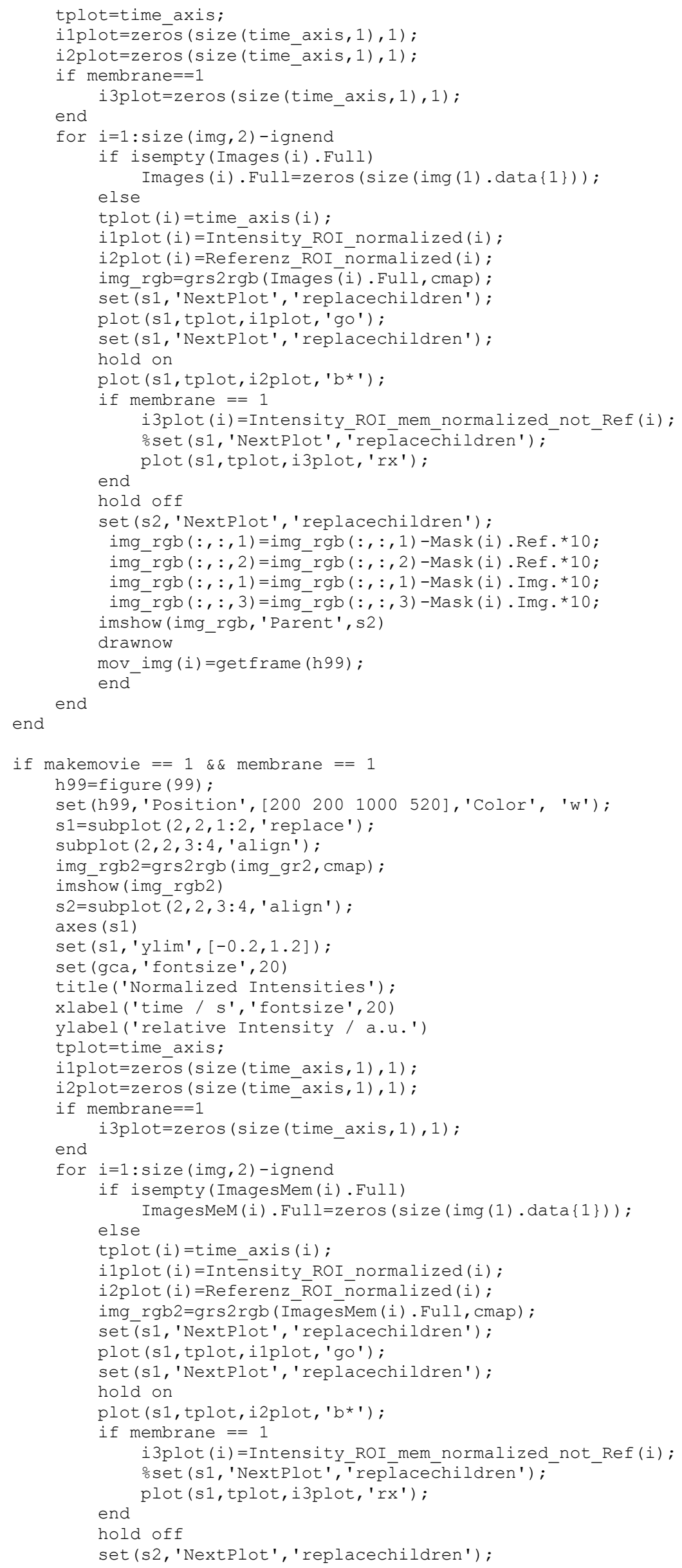




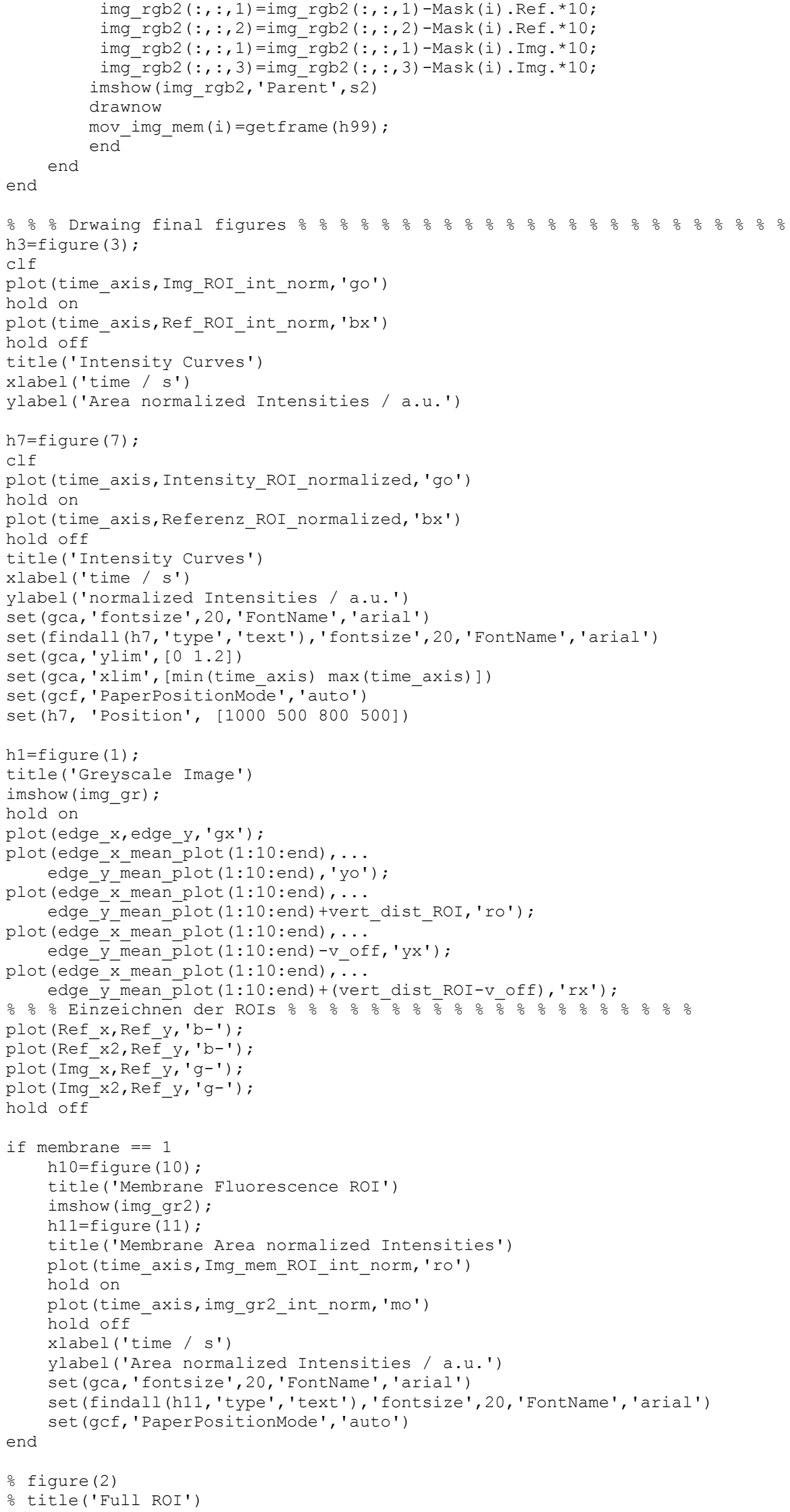

응 figure (2)

otitle('Full ROI') 


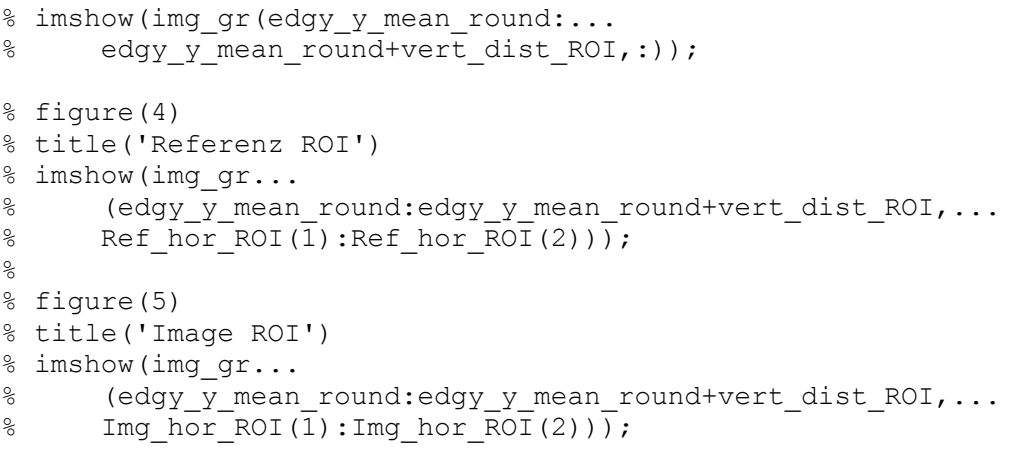

h6=figure $(6)$;

title ('Image')

imshow(img_gr);

drawnow

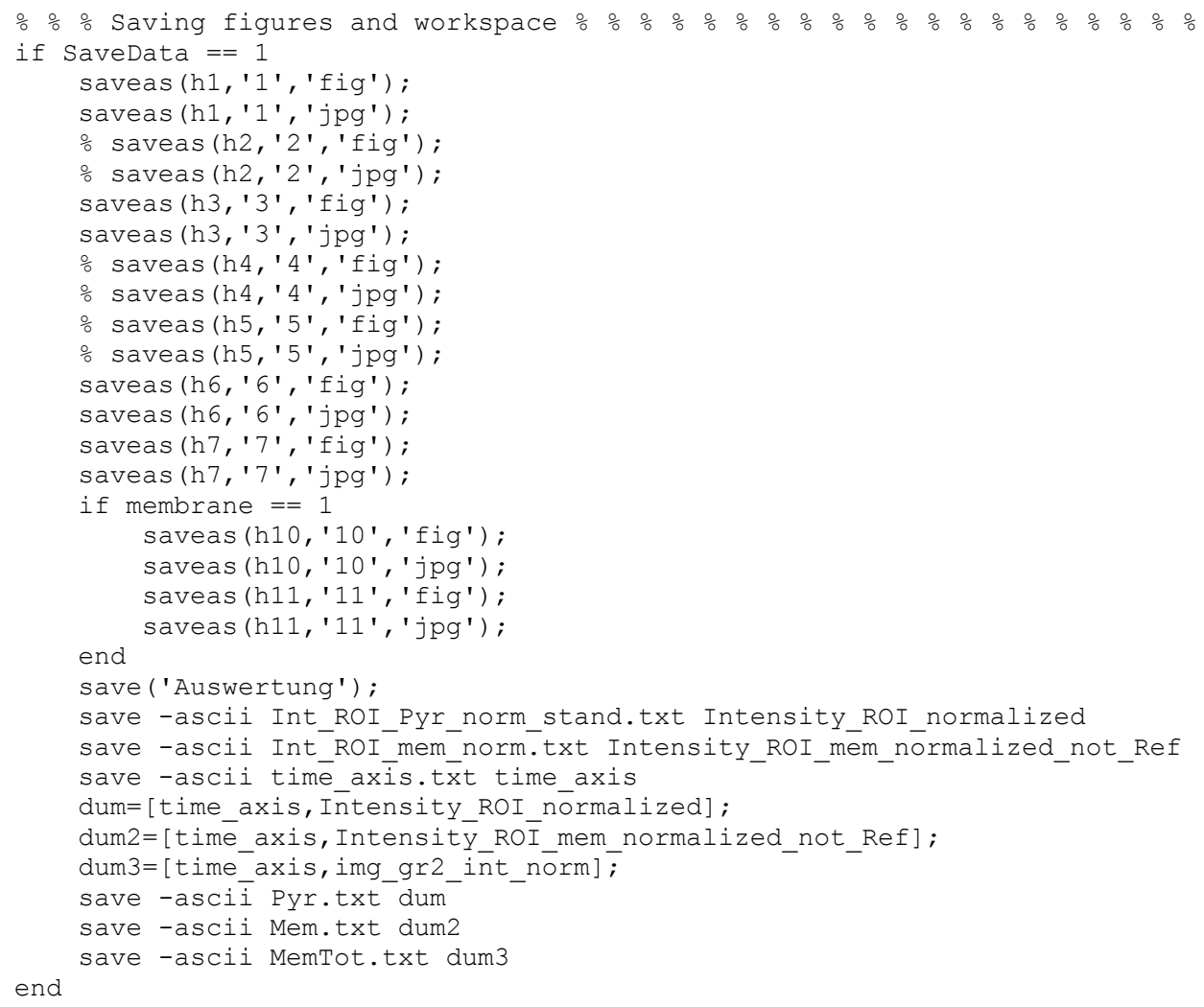




\section{Acknowledgements}

This work would not have been completed without the help of a number of people whom I would like to thank here.

First of all, I would like to thank my supervisor Prof. Claudia Steinem for the opportunity to carry out my dissertation in her group, for providing the research topic, her supervision and advice throughout the work and her patience and constructive feedback during completion.

I would also like to thank all members of the Steinem lab for the good working climate and their collegiality. A special thanks goes to Dr. Ingo Mey for his personal and professional support and advice. Dr. Mey generously provided the MATLAB script used to analyze imaging data in this work. The Janshoff lab which shared work projects on several issues also deserves a special mention.

Furthermore, I would like to thank my thesis committee members Prof. Reinhard Jahn and Prof. Jörg Enderlein for their constructive input and their guidance throughout my research advances. Thank you to the additional members of my examination board, Prof. Bert de Groot, Dr. Jochen Hub and Dr. Hans Dieter Schmitt, and for taking the time to review this thesis.

I want to thank the GGNB PBCS office for providing continuous support throughout my work, and the GGNB graduate school in general for the opportunities to grow and develop during courses, events, and excursions.

My friends have always been an important part of my life to balance work, by helping me with their advice and by being there for me, I am very thankful for having you! I especially want to thank my girlfriend Saskia for her input, for proofreading this manuscript and for her emotional support.

Finally, a big thank you goes to my family who have always supported me and believed in me. I could never have done it without you! 


\section{References}

1. Zhang, M.; Zhao, J.; Zheng, J. Molecular understanding of a potential functional link between antimicrobial and amyloid peptides, Soft matter. 2014 10, 7425-7451.

2. Neubacher, H.; Mey, I.; Carnarius, C.; Lazzara, T. D.; Steinem, C. Permeabilization assay for antimicrobial peptides based on pore-spanning lipid membranes on nanoporous alumina, Langmuir. 2014 30, 4767-4774.

3. Chan, D. I.; Prenner, E. J.; Vogel, H. J. Tryptophan- and arginine-rich antimicrobial peptides: structures and mechanisms of action, Biochimica et biophysica acta. 2006 1758, 1184-1202.

4. Spellberg, B.; Powers, J. H.; Brass, E. P.; Miller, L. G.; Edwards, J. E. JR. Trends in antimicrobial drug development: implications for the future, Clinical infectious diseases: an official publication of the Infectious Diseases Society of America. 2004 38, 1279-1286.

5. Zasloff, M. Antimicrobial peptides of multicellular organisms, Nature. 2002 415, 389395.

6. Brogden K. A. Antimicrobial peptides: Pore formers or metabolic inhibitors in bacteria?, Nat. Rev. Microbiol. 2005 3, 238.

7. Yeaman, M. R.; Yount, N. Y. Mechanisms of antimicrobial peptide action and resistance, Pharmacological reviews. 2003 55, 27-55.

8. Abraham, E. P.; Chain, E. An Enzyme from Bacteria able to Destroy Penicillin, Nature. $1940146,837$.

9. Dubos, R. J. Studies on a bactericidal agent extracted from a soil bacillus ACTIVITY OF A PROTEIN-FREE FRACTION, Journal of Experimental Medicine. 1939 70, 249-256.

10. Tyrosur. Engelhard Pharma, Niederdorfelden, Germany. Available at www.tyrosur.de.

11. MEDICE Arzneimittel Pütter. Available at www.dorithricin.de.

12. Hoskin, D. W.; Ramamoorthy, A. Studies on anticancer activities of antimicrobial peptides, Biochimica et biophysica acta. 2008 1778, 357-375.

13. Hancock, R. E. W. Peptide antibiotics, The Lancet. 1997 349, 418-422.

14. Dziarski, R. Peptidoglycan recognition proteins (PGRPs), Molecular immunology. 2004 40, 877-886.

15. Garver, L. S.; Wu, J.; Wu, L. P. The peptidoglycan recognition protein PGRP-SC1a is essential for Toll signaling and phagocytosis of Staphylococcus aureus in Drosophila, 
Proceedings of the National Academy of Sciences of the United States of America. 2006 103, 660-665.

16. Tossi, A.; Sandri, L.; Giangaspero, A. Amphipathic, alpha-helical antimicrobial peptides, Biopolymers. 2000 55, 4-30.

17. Melo, M. N.; Ferre, R.; Castanho, Miguel A R B. Antimicrobial peptides: linking partition, activity and high membrane-bound concentrations, Nat. Rev. Microbiol. 2009 , $245-250$.

18. Wachinger, M. et al. Antimicrobial peptides melittin and cecropin inhibit replication of human immunodeficiency virus 1 by suppressing viral gene expression, The Journal of general virology. 199879 ( Pt 4), 731-740.

19. Matsuzaki, K. Why and how are peptide-lipid interactions utilized for self-defense? Magainins and tachyplesins as archetypes, Biochimica et biophysica acta 1999 1462, 110.

20. Tomasinsig, L. et al. Role of Cathelicidin Peptides in Bovine Host Defense and Healing, Probiotics and antimicrobial proteins. 2010 2, 12-20.

21. Teixeira, V.; Feio, M. J.; Bastos, M. Role of lipids in the interaction of antimicrobial peptides with membranes, Progress in lipid research. 2012 51, 149-177.

22. Gause, G. F.; Brazhnikova, M. G. Gramicidin S and its use in the Treatment of Infected Wounds, Nature. 1944 154, 703.

23. Falla, T. J.; Karunaratne, D. N.; Hancock, R. E. Mode of action of the antimicrobial peptide indolicidin, The Journal of biological chemistry. 1996 271, 19298-19303.

24. Mochon, A. B.; Liu, H. The antimicrobial peptide histatin-5 causes a spatially restricted disruption on the Candida albicans surface, allowing rapid entry of the peptide into the cytoplasm, PLoS pathogens. 2008 4, e1000190.

25. Rozek, A.; Friedrich, C. L.; Hancock, R. E. Structure of the bovine antimicrobial peptide indolicidin bound to dodecylphosphocholine and sodium dodecyl sulfate micelles, Biochemistry. 2000 39, 15765-15774.

26. Chan, Y. R.; Zanetti, M.; Gennaro, R.; Gallo, R. L. Anti-microbial activity and cell binding are controlled by sequence determinants in the anti-microbial peptide PR-39, The Journal of investigative dermatology. 2001 116, 230-235.

27. Diamond, G.; Beckloff, N.; Weinberg, A.; Kisich, K. O. The roles of antimicrobial peptides in innate host defense, Current pharmaceutical design. 2009 15, 2377-2392.

28. Robinson, W. E.; McDougall, B.; Tran, D.; Selsted, M. E. Anti-HIV-1 activity of indolicidin, an antimicrobial peptide from neutrophils, Journal of leukocyte biology. 1998 63, 94-100. 
29. Albiol Matanic, V. C.; Castilla, V. Antiviral activity of antimicrobial cationic peptides against Junin virus and herpes simplex virus, International journal of antimicrobial agents. 2004 23, 382-389.

30. Henriques, S. T.; Melo, M. N.; Castanho, Miguel A R B. Cell-penetrating peptides and antimicrobial peptides: how different are they?, The Biochemical journal. 2006 399, 1-7.

31. Lohner, K.; Latal, A.; Degovics, G.; Garidel, P. Packing characteristics of a model system mimicking cytoplasmic bacterial membranes, Chemistry and physics of lipids. 2001 111, 177-192.

32. Pozo Navas, B. et al. Composition dependence of vesicle morphology and mixing properties in a bacterial model membrane system, Biochimica et biophysica acta. 2005 1716, $40-48$.

33. Yount, N. Y.; Bayer, A. S.; Xiong, Y. Q.; Yeaman, M. R. Advances in antimicrobial peptide immunobiology, Biopolymers. 2006 84, 435-458.

34. Huang, H. W. Action of antimicrobial peptides: two-state model, Biochemistry. 200039 , 8347-8352.

35. Bastos, M. et al. Energetics and partition of two cecropin-melittin hybrid peptides to model membranes of different composition, Biophysical journal. 2008 94, 2128-2141.

36. Sharma, S.; Sahoo, N.; Bhunia, A. Antimicrobial Peptides and their Pore/Ion Channel Properties in Neutralization of Pathogenic Microbes, Current topics in medicinal chemistry. 2016 16, 46-53.

37. Pouny, Y.; Rapaport, D.; Mor, A.; Nicolas, P.; Shai, Y. Interaction of antimicrobial dermaseptin and its fluorescently labeled analogues with phospholipid membranes, Biochemistry. 1992 31, 12416-12423.

38. Wimley, W. C.; White, S. H. Experimentally determined hydrophobicity scale for proteins at membrane interfaces, Nature structural biology. 1996 3, 842-848.

39. White, S. H.; Wimley, W. C. Hydrophobic interactions of peptides with membrane interfaces, Biochimica et biophysica acta. 1998 1376, 339-352.

40. Nguyen, L. T.; Haney, E. F.; Vogel, H. J. The expanding scope of antimicrobial peptide structures and their modes of action, Trends in biotechnology. 2011 29, 464-472.

41. Baumann, G.; Mueller, P. A molecular model of membrane excitability, Journal of supramolecular structure. 1974 2, 538-557.

42. Ludtke, S. J. et al. Membrane pores induced by magainin, Biochemistry. 1996 35, 1372313728.

43. Leontiadou, H.; Mark, A. E.; Marrink, S. J. Antimicrobial peptides in action, Journal of the American Chemical Society. 2006 128, 12156-12161. 
44. Grage, S. L.; Afonin, S.; Kara, S.; Buth, G.; Ulrich, A. S. Membrane Thinning and Thickening Induced by Membrane-Active Amphipathic Peptides, Frontiers in cell and developmental biology. 2016 4, 65.

45. Huang, H. W. Molecular mechanism of antimicrobial peptides: the origin of cooperativity, Biochimica et biophysica acta. 2006 1758, 1292-1302.

46. Khandelia, H.; Ipsen, J. H.; Mouritsen, O. G. The impact of peptides on lipid membranes, Biochimica et biophysica acta. 2008 1778, 1528-1536.

47. Grage, S. L.; Afonin, S.; Ulrich, A. S. Dynamic transitions of membrane-active peptides, Methods in molecular biology (Clifton, N.J.). 2010 618, 183-207.

48. Bertelsen, K.; Dorosz, J.; Hansen, S. K.; Nielsen, N. C.; Vosegaard, T. Mechanisms of peptide-induced pore formation in lipid bilayers investigated by oriented $31 \mathrm{P}$ solid-state NMR spectroscopy, PloS one. 2012 7, e47745.

49. Fernandez-Reyes, M. et al. Lysine N(epsilon)-trimethylation, a tool for improving the selectivity of antimicrobial peptides, Journal of medicinal chemistry. 2010 53, 5587-5596.

50. Zasloff, M. Magainins, a class of antimicrobial peptides from Xenopus skin: isolation, characterization of two active forms, and partial cDNA sequence of a precursor, Proceedings of the National Academy of Sciences of the United States of America. 1987 84, 5449-5453.

51. Matsuzaki, K. et al. Magainin 1-induced leakage of entrapped calcein out of negativelycharged lipid vesicles, Biochimica et biophysica acta. 1989 981, 130-134.

52. Williams, R. W. et al. Raman spectroscopy of synthetic antimicrobial frog peptides magainin 2a and PGLa, Biochemistry. 1990 29, 4490-4496.

53. Bechinger, B.; Zasloff, M.; Opella, S. J. Structure and orientation of the antibiotic peptide magainin in membranes by solid-state nuclear magnetic resonance spectroscopy, Protein science : a publication of the Protein Society. 1993 2, 2077-2084.

54. Mahalka, A. K.; Kinnunen, P. K. J. Binding of amphipathic alpha-helical antimicrobial peptides to lipid membranes: lessons from temporins B and L, Biochimica et biophysica acta. 2009 1788, 1600-1609.

55. Zhao, H. et al. Interaction of the antimicrobial peptide pheromone Plantaricin A with model membranes: implications for a novel mechanism of action, Biochimica et biophysica acta. 2006 1758, 1461-1474.

56. Matsuzaki, K. et al. Relationship of membrane curvature to the formation of pores by magainin 2, Biochemistry. 1998 37, 11856-11863.

57. Terwilliger, T. C.; Eisenberg, D. The structure of melittin. II. Interpretation of the structure, The Journal of biological chemistry. 1982 257, 6016-6022. 
58. Frey, S.; Tamm, L. K. Orientation of melittin in phospholipid bilayers. A polarized attenuated total reflection infrared study, Biophysical journal. 1991 60, 922-930.

59. Cornut, I.; Desbat, B.; Turlet, J. M.; Dufourcq, J. In situ study by polarization modulated Fourier transform infrared spectroscopy of the structure and orientation of lipids and amphipathic peptides at the air-water interface, Biophysical journal. 1996 70, 305-312.

60. Gough, M.; Hancock, R. E.; Kelly, N. M. Antiendotoxin activity of cationic peptide antimicrobial agents, Infection and immunity. 1996 64, 4922-4927.

61. Matsuzaki, K.; Yoneyama, S.; Miyajima, K. Pore formation and translocation of melittin, Biophysical journal. 1997 73, 831-838.

62. Maria-Neto, S.; Almeida, K. C. de; Macedo, M. L. R.; Franco, O. L. Understanding bacterial resistance to antimicrobial peptides: From the surface to deep inside, Biochimica et biophysica acta. 2015 1848, 3078-3088.

63. van den Bogaart, G.; Mika, J. T.; Krasnikov, V.; Poolman, B. The lipid dependence of melittin action investigated by dual-color fluorescence burst analysis, Biophysical journal. 2007 93, 154-163.

64. van den Bogaart G.; Guzmán J. V.; Mika J. T.; Poolman B. On the mechanism of pore formation by melittin, J. Biol. Chem. 2008 283, 33854.

65. Kokot, G.; Mally, M.; Svetina, S. The dynamics of melittin-induced membrane permeability, European biophysics journal : EBJ. 2012 41, 461-474.

66. Janshoff, A.; Steinem, C. Transport across artificial membranes-an analytical perspective, Analytical and bioanalytical chemistry. 2006 385, 433-451.

67. Tamm, L. K.; McConnell, H. M. Supported phospholipid bilayers, Biophysical journal. 1985 47, 105-113.

68. Johnson, S. J. et al. Structure of an adsorbed dimyristoylphosphatidylcholine bilayer measured with specular reflection of neutrons, Biophysical journal. 1991 59, 289-294.

69. Brozell, A. M.; Muha, M. A.; Sanii, B.; Parikh, A. N. A class of supported membranes: formation of fluid phospholipid bilayers on photonic band gap colloidal crystals, Journal of the American Chemical Society. 2006 128, 62-63.

70. Dertinger, T.; Hocht, I. von der; Benda, A.; Hof, M.; Enderlein, J. Surface sticking and lateral diffusion of lipids in supported bilayers, Langmuir. 2006 22, 9339-9344.

71. Tanaka, M.; Sackmann, E. Polymer-supported membranes as models of the cell surface, Nature. 2005 437, 656-663.

72. Mueller, P.; Rudin, D. O.; Tien, H. T.; Wescott, W. C. Reconstitution of cell membrane structure in vitro and its transformation into an excitable system, Nature. 1962 194, 979980 . 
73. Mueller, P.; Rudin, D. O.; Tien, H. T.; Wescott, W. C. Methods for the formation of single bimolecular lipid membranes in aqueous solution, J. Phys. Chem. 1963 67, 534-535.

74. Castellana, E. T.; Cremer, P. S. Solid supported lipid bilayers. From biophysical studies to sensor design, Surface Science Reports. 2006 61, 429-444.

75. Reimhult, E.; Kumar, K. Membrane biosensor platforms using nano- and microporous supports, Trends in biotechnology. 2008 26, 82-89.

76. Kaufeld, T.; Steinem, C.; Schmidt, C. F. Microporous device for local electric recordings on model lipid bilayers, J. Phys. D: Appl. Phys. 2015 48, 025401.

77. Weiskopf D.; Schmitt E. K.; Klühr M. H.; Dertinger S. K.; Steinem C. Micro-BLMs on highly ordered porous silicon substrates: Rupture process and lateral mobility, Langmuir. $200723,9134$.

78. Mey, I.; Steinem, C.; Janshoff, A. Biomimetic functionalization of porous substrates. Towards model systems for cellular membranes, J. Mater. Chem. 2012 22, 19348.

79. Heinemann, F.; Schwille, P. Preparation of micrometer-sized free-standing membranes, Chemphyschem : a European journal of chemical physics and physical chemistry. 2011 12, 2568-2571.

80. Schmitt, E. K.; Nurnabi, M.; Bushby, R. J.; Steinem, C. Electrically insulating poresuspending membranes on highly ordered porous alumina obtained from vesicle spreading, Soft matter. 2008 4, 250-253.

81. Schmitt, E. K.; Weichbrodt, C.; Steinem, C. Impedance analysis of gramicidin D in poresuspending membranes, Soft matter. 2009 5, 3347.

82. Schwenen, L. L. G. et al. Resolving single membrane fusion events on planar porespanning membranes, Scientific reports. 2015 5, 12006.

83. Höfer, I.; Steinem, C. A membrane fusion assay based on pore-spanning lipid bilayers, Soft matter. 2011 7, 1644.

84. Römer, W.; Steinem, C. Impedance Analysis and Single-Channel Recordings on NanoBlack Lipid Membranes Based on Porous Alumina, Biophysical journal. 2004 86, 955965.

85. Schmitt, E. K.; Vrouenraets, M.; Steinem, C. Channel activity of OmpF monitored in nano-BLMs, Biophysical journal. 2006 91, 2163-2171.

86. Frese, D.; Steltenkamp, S.; Schmitz, S.; Steinem, C. In situ generation of electrochemical gradients across pore-spanning membranes, RSC Adv. 2013 3, 15752.

87. Gasteiger, E. ExPASy. The proteomics server for in-depth protein knowledge and analysis, Nucleic Acids Research. 2003 31, 3784-3788. 
88. Matsuzaki, K. Magainins as paradigm for the mode of action of pore forming polypeptides, Biochimica et Biophysica Acta (BBA) - Reviews on Biomembranes. 1998 1376, 391-400.

89. Angelova, M. I.; Dimitrov, D. S. Liposome electroformation, Faraday Discuss. Chem. Soc. $198681,303$.

90. Angelova, M. I.; Soléau, S.; Méléard, P.; Faucon, F.; Bothorel, P. in Trends in Colloid and Interface Science VI, edited by C. Helm, M. Lösche \& H. Möhwald (Steinkopff, Darmstadt, 1992), pp. 127-131.

91. Lazzara, T. D.; Carnarius, C.; Kocun, M.; Janshoff, A.; Steinem, C. Separating attolitersized compartments using fluid pore-spanning lipid bilayers, ACS Nano. 2011 5, 69356944.

92. O'Sullivan, J. P.; Wood, G. C. The Morphology and Mechanism of Formation of Porous Anodic Films on Aluminium, Proceedings of the Royal Society A: Mathematical, Physical and Engineering Sciences. 1970 317, 511-543.

93. Masuda, H.; Fukuda, K. Ordered metal nanohole arrays made by a two-step replication of honeycomb structures of anodic alumina, Science (New York, N.Y.). 1995 268, 14661468.

94. Lazzara, T. D. Dissertation: Directing macromolecular assemblies by tailored surface functionalizations of nanoporous alumina, Georg-August-Universität Göttingen.

95. Kocun, M.; Lazzara, T. D.; Steinem, C.; Janshoff, A. Preparation of solvent-free, porespanning lipid bilayers: modeling the low tension of plasma membranes, Langmuir. 2011 27, 7672-7680.

96. Singh, J.; Whitten, J. E. Adsorption of 3-Mercaptopropyltrimethoxysilane on Silicon Oxide Surfaces and Adsorbate Interaction with Thermally Deposited Gold, J. Phys. Chem. C. 2008 112, 19088-19096.

97. Carnarius, C. Functional reconstitution of connexons in artificial membranes: expression, purification and characterization of connexin 43, Georg-August-Universität Göttingen, 2012.

98. Frese, D. Dissertation: Phospholipid membranes on microporous substrates: in situ generation of electrochemical gradients, Georg-August-Universität Göttingen, 2013.

99. Gleisner, M. et al. Driving a planar model system into the 3(rd) dimension: generation and control of curved pore-spanning membrane arrays, Soft matter. 2014 10, 6228-6236.

100. Kuhlmann, J. W.; Mey, I. P.; Steinem, C. Modulating the lateral tension of solventfree pore-spanning membranes, Langmuir. 2014 30, 8186-8192. 
101. Melo, M. N.; Castanho, Miguel A R B. Omiganan interaction with bacterial membranes and cell wall models. Assigning a biological role to saturation, Biochimica et biophysica acta. 2007 1768, 1277-1290.

102. Pistolesi, S.; Pogni, R.; Feix, J. B. Membrane insertion and bilayer perturbation by antimicrobial peptide CM15, Biophysical journal. 2007 93, 1651-1660.

103. Pott, T.; Paternostre, M.; Dufourc, E. J. A comparative study of the action of melittin on sphingomyelin and phosphatidylcholine bilayers, European Biophysics Journal. 1998 27, 237-245.

104. Weiss, T. M. et al. Two States of Cyclic Antimicrobial Peptide RTD-1 in Lipid Bilayers, Biochemistry. 2002 41, 10070-10076.

105. Wenk, M. R.; Seelig, J. Magainin 2 amide interaction with lipid membranes: calorimetric detection of peptide binding and pore formation, Biochemistry. 1998 37, 3909-3916.

106. Papo, N.; Shai, Y. Can we predict biological activity of antimicrobial peptides from their interactions with model phospholipid membranes?, Peptides. 2003 24, 1693-1703.

107. Saßen, C. Dissertation: A scanning ion conductance microscopy assay to investigate interactions between cell penetrating peptides and pore-suspending membranes, GeorgAugust-Universität Göttingen, 2013.

108. Ladokhin, A. S.; White, S. H. 'Detergent-like' permeabilization of anionic lipid vesicles by melittin, Biochimica et Biophysica Acta (BBA) - Biomembranes. 2001 1514, $253-260$.

109. Chen, Y. et al. Rational design of alpha-helical antimicrobial peptides with enhanced activities and specificity/therapeutic index, The Journal of biological chemistry. 2005280, $12316-12329$.

110. Oren, Z.; Shai, Y. Selective lysis of bacteria but not mammalian cells by diastereomers of melittin: structure-function study, Biochemistry. 1997 36, 1826-1835.

111. Fox, J. L. Antimicrobial peptides stage a comeback, Nature biotechnology. 2013 31, 379-382.

112. Malanovic, N.; Lohner, K. Gram-positive bacterial cell envelopes: The impact on the activity of antimicrobial peptides, Biochimica et biophysica acta. 2016 1858, 936-946.

113. Liu, S.; Fan, L.; Sun, J.; Lao, X.; Zheng, H. Computational resources and tools for antimicrobial peptides, Journal of peptide science : an official publication of the European Peptide Society. 2016.

114. da Cunha, N. B. et al. The next generation of antimicrobial peptides (AMPs) as molecular therapeutic tools for the treatment of diseases with social and economic impacts, Drug discovery today. 2016. 
115. Orth, A.; Johannes, L.; Romer, W.; Steinem, C. Creating and modulating microdomains in pore-spanning membranes, Chemphyschem : a European journal of chemical physics and physical chemistry. 2012 13, 108-114.

116. Shabardina, V. et al. Mode of Ezrin-Membrane Interaction as a Function of PIP2 Binding and Pseudophosphorylation, Biophysical journal. 2016 110, 2710-2719.

117. Ludolphs, M. et al. Specificity of Collybistin-Phosphoinositide Interactions: IMPACT OF THE INDIVIDUAL PROTEIN DOMAINS, The Journal of biological chemistry. $2016291,244-254$.

118. Lazzara, T. D.; Behn, D.; Kliesch, T.-T.; Janshoff, A.; Steinem, C. Phospholipids as an alternative to direct covalent coupling: surface functionalization of nanoporous alumina for protein recognition and purification, Journal of colloid and interface science. 2012 366, 57-63.

119. Lazzara, T. D.; Mey, I.; Steinem, C.; Janshoff, A. Benefits and limitations of porous substrates as biosensors for protein adsorption, Analytical chemistry. 2011 83, 5624-5630.

120. Mager, M. D.; Melosh, N. A. Nanopore-Spanning Lipid Bilayers for Controlled Chemical Release, Adv. Mater. 2008 20, 4423-4427.

121. Korman, C. E.; Megens, M.; Ajo-Franklin, C. M.; Horsley, D. A. Nanopore-spanning lipid bilayers on silicon nitride membranes that seal and selectively transport ions, Langmuir. 2013 29, 4421-4425.

122. Urban, M.; Vor der Bruggen, M.; Tampe, R. Membrane Transport Processes Analyzed by a Highly Parallel Nanopore Chip System at Single Protein Resolution, Journal of visualized experiments : JoVE. 2016.

123. Fine, T. et al. Elasticity mapping of apical cell membranes, Soft matter. 2009 5, 3262.

124. Lorenz, B. et al. Elasticity mapping of pore-suspending native cell membranes, Small (Weinheim an der Bergstrasse, Germany). 2009 5, 832-838.

125. Im, H.; Wittenberg, N. J.; Lesuffleur, A.; Lindquist, N. C.; Oh, S.-H. Membrane protein biosensing with plasmonic nanopore arrays and pore-spanning lipid membranes, Chemical science. 2010 1, 688-696.

126. Kresák, S.; Hianik, T.; Naumann, R. L. C. Giga-seal solvent-free bilayer lipid membranes. From single nanopores to nanopore arrays, Soft matter. 2009 5, 4021.

127. Schutte, O. M. et al. 2-Hydroxy Fatty Acid Enantiomers of Gb3 Impact Shiga Toxin Binding and Membrane Organization, Biophysical journal. 2015 108, 2775-2778.

128. Weiß, K. et al. Quantifying the Diffusion of Membrane Proteins and Peptides in Black Lipid Membranes with 2-Focus Fluorescence Correlation Spectroscopy, Biophysical journal. 2013 105, 455-462. 
129. Mey, I. et al. Local membrane mechanics of pore-spanning bilayers, Journal of the American Chemical Society. 2009 131, 7031-7039.

130. Steltenkamp, S. et al. Mechanical properties of pore-spanning lipid bilayers probed by atomic force microscopy, Biophysical journal. 2006 91, 217-226.

131. Goncalves, R. P. et al. Two-chamber AFM: probing membrane proteins separating two aqueous compartments, Nature methods. 2006 3, 1007-1012.

132. White, G. F.; Racher, K. I.; Lipski, A.; Hallett, F.; Wood, J. M. Physical properties of liposomes and proteoliposomes prepared from Escherichia coli polar lipids, Biochimica et Biophysica Acta (BBA) - Biomembranes. 2000 1468, 175-186.

133. Murzyn, K.; Rog, T.; Pasenkiewicz-Gierula, M. Phosphatidylethanolaminephosphatidylglycerol bilayer as a model of the inner bacterial membrane, Biophysical journal. 2005 88, 1091-1103.

134. Zhao, W.; Rog, T.; Gurtovenko, A. A.; Vattulainen, I.; Karttunen, M. Role of phosphatidylglycerols in the stability of bacterial membranes, Biochimie. 2008 90, 930938.

135. Epand, R. M.; Rotem, S.; Mor, A.; Berno, B.; Epand, R. F. Bacterial membranes as predictors of antimicrobial potency, Journal of the American Chemical Society. 2008130, 14346-14352.

136. Murzyn, K.; Rog, T.; Pasenkiewicz-Gierula, M. Phosphatidylethanolaminephosphatidylglycerol bilayer as a model of the inner bacterial membrane, Biophysical journal. 2005 88, 1091-1103.

137. Glukhov, E.; Stark, M.; Burrows, L. L.; Deber, C. M. Basis for selectivity of cationic antimicrobial peptides for bacterial versus mammalian membranes, The Journal of biological chemistry. 2005 280, 33960-33967.

138. Sood, R.; Kinnunen, P. K. J. Cholesterol, lanosterol, and ergosterol attenuate the membrane association of LL-37(W27F) and temporin L, Biochimica et biophysica acta. 2008 1778, 1460-1466.

139. Friedrich, C. L.; Moyles, D.; Beveridge, T. J.; Hancock, R. E. Antibacterial action of structurally diverse cationic peptides on gram-positive bacteria, Antimicrobial agents and chemotherapy. 2000 44, 2086-2092.

140. Guina, T.; Yi, E. C.; Wang, H.; Hackett, M.; Miller, S. I. A PhoP-regulated outer membrane protease of Salmonella enterica serovar typhimurium promotes resistance to alpha-helical antimicrobial peptides, Journal of bacteriology. 2000 182, 4077-4086.

141. Ramu, P. et al. The surface protease PgtE of Salmonella enterica affects complement activity by proteolytically cleaving C3b, C4b and C5, FEBS letters. 2007 581, 1716-1720. 
142. Ulvatne, H.; Haukland, H. H.; Samuelsen, Ø.; Krämer, M.; Vorland, L. H. Proteases in Escherichia coli and Staphylococcus aureus confer reduced susceptibility to lactoferricin $\mathrm{B}$, The Journal of antimicrobial chemotherapy. 2002 50, 461-467.

143. Shafer, W. M.; Qu, X.; Waring, A. J.; Lehrer, R. I. Modulation of Neisseria gonorrhoeae susceptibility to vertebrate antibacterial peptides due to a member of the resistance/nodulation/division efflux pump family, Proceedings of the National Academy of Sciences of the United States of America. 1998 95, 1829-1833.

144. Bengoechea, J. A.; Skurnik, M. Temperature-regulated efflux pump/potassium antiporter system mediates resistance to cationic antimicrobial peptides in Yersinia, Molecular microbiology. 2000 37, 67-80.

145. Kupferwasser, L. I. et al. Plasmid-mediated resistance to thrombin-induced platelet microbicidal protein in staphylococci: role of the qacA locus, Antimicrobial agents and chemotherapy. 1999 43, 2395-2399.

146. Bina, X. R.; Provenzano, D.; Nguyen, N.; Bina, J. E. Vibrio cholerae RND family efflux systems are required for antimicrobial resistance, optimal virulence factor production, and colonization of the infant mouse small intestine, Infection and immunity. 2008 76, 3595-3605.

147. Kang, H.-K.; Kim, C.; Seo, C. H.; Park, Y. The therapeutic applications of antimicrobial peptides (AMPs): a patent review, Journal of microbiology (Seoul, Korea). 2017 55, 1-12.

148. Zhang, L.; Rozek, A.; Hancock, R. E. Interaction of cationic antimicrobial peptides with model membranes, The Journal of biological chemistry. 2001 276, 35714-35722.

149. Giacometti, A.; Cirioni, O.; Greganti, G.; Quarta, M.; Scalise, G. In vitro activities of membrane-active peptides against gram-positive and gram-negative aerobic bacteria, Antimicrobial agents and chemotherapy. 1998 42, 3320-3324.

150. Rosenfeld, Y.; Barra, D.; Simmaco, M.; Shai, Y.; Mangoni, M. L. A synergism between temporins toward Gram-negative bacteria overcomes resistance imposed by the lipopolysaccharide protective layer, The Journal of biological chemistry. $2006 \mathbf{2 8 1}$, 28565-28574.

151. Giacometti, A. et al. Comparative activities of cecropin A, melittin, and cecropin Amelittin peptide $\mathrm{CA}(1-7) \mathrm{M}(2-9) \mathrm{NH} 2$ against multidrug-resistant nosocomial isolates of Acinetobacter baumannii, Peptides. 2003 24, 1315-1318.

152. Marr, A. K.; Gooderham, W. J.; Hancock, R. E. Antibacterial peptides for therapeutic use: obstacles and realistic outlook, Current opinion in pharmacology. 2006 6, 468-472. 


\section{Curriculum Vitae}

\section{Henrik Neubacher}

Date of birth $\quad$ May $4^{\text {th }} 1983$

Place of birth Minden/Westfalen

E-mail henrikneubacher@gmx.de

2010-2017Ｇeorg-August-Universität Göttingen

Göttingen Graduate School for Neurosciences, Biophysics, and Molecular Biosciences (GGNB)

Member of the doctoral program "Physics of Biological and Complex Systems"

2010-2015Ｇeorg-August-Universität Göttingen

Institute for Organic and Biomolecular Chemistry

Doctoral thesis "Pore-spanning lipid membranes as a tool to study membrane permeabilization by antimicrobial peptides “

2003-2009Ｌeibniz Universität Hannover

Chemistry studies / Diplom

Thesis / Diplomarbeit „Etablierung der Produktionsbedingungen für die rekombinante Expression und Aufreinigung des Wachstumsfaktors hLIF in Escherichia coli “ at the Institute for Technical Chemistry

2002-2003 Paritätische Sozialdienste Minden-Lübbecke

Civilian Service

Individual care of severely handicapped patients

\section{High School Gymnasium Stolzenau}

University entry exam / Abitur 